\title{
Run05 Proton Beam Polarization Measurements by pC-Polarimeter (ver. 1.1)
}

I. Nakagawa, I. Alekseev, A. Bazilevsky, A. Bravar, G. Bunce, S. Dhawan, K.O. Eyser, R. Gill, W. Haeberli, H. Huang, Y. Makdisi, A. Nass, H. Okada, E. Stephenson, D.N. Svirida, T. Wise, J. Wood, K. Yip, A. Zelenski

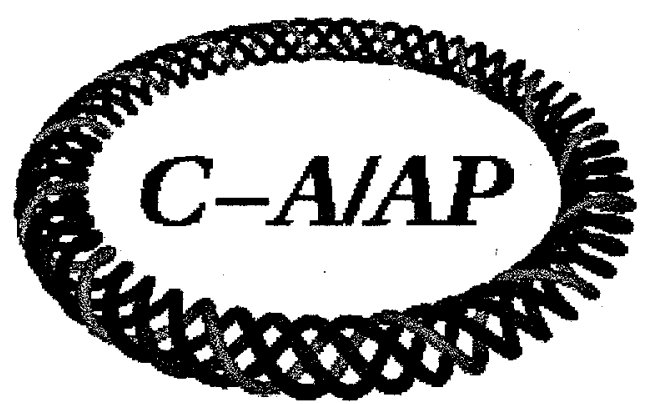

\section{Collider-Accelerator Department Brookhaven National Laboratory Upton, NY 11973}

Notice: This document has been authorized by employees of Brookhaven Science Associates, LLC under Contract No. DE-AC02-98CH10886 with the U.S. Department of Energy. The United States Government retains a non-exclusive, paid-up, irrevocable, world-wide license to publish or reproduce the published form of this document, or allow others to do so, for United States Government purposes. 


\title{
Run05 Proton Beam Polarization Measurements by pC-Polarimeter (ver.1.1)
}

\author{
I. Nakagawa \\ RIKEN institute, Hirosawa, Wako, 351-0198, Saitama, Japan \\ I. Alekseev, D.N. Svirida
}

Institute for Theoretical and Experimental Physics, 117259 Moscow, Russia

A. Bazilevsky, G. Bunce, R. Gill, H. Huang, Y. Makdisi, H. Okada,

J. Wood, K. Yip, A. Zelenski

Brookhaven National Laboratory, Upton, NY 11973, USA

A. Bravar

University of Geneva, 1205 Geneva, Switzerland

S. Dhawan

Yale University, New Haven, CT 06520, USA

K.O. Eyser

University of California, Riverside, CA 92521, USA

W. Haeberli, T. Wise

University of Wisconsin, WI 53706, USA

A. Nass,

University of Erlangen, 91058 Erlangen, Germany

and

E. Stephenson

Indiana University Cyclotron Facility, Bloomington, IN 47408, USA

June 10, 2008 


\section{Contents}

1 INTRODUCTION

2 EXPERIMENTAL APPARATUS 5

2.1 Target System And Detectors . . . . . . . . . . . . . . . . 5

2.2 Readout Electronics And Data Acquisition . . . . . . . . . . . . . . 7

3 CALIBRATION AND ENERGY CORRECTION 13

3.1 Energy Calibration . . . . . . . . . . . . . . . . . . 13

3.2 Effective Dead-Layer and Energy Correction . . . . . . . . . . . . . . 15

3.3 Stability And Dependency . . . . . . . . . . . . . . . . . . . . . 20

3.3.1 Peak Beam Intensity Dependence . . . . . . . . . . . . . . . 22

3.3.2 Average Event Rate Dependence . . . . . . . . . . . . . . . . . 23

3.3.3 56 and 112 Bunch Modes Effect . . . . . . . . . . . . . . 28

3.3 .4 Drifting $t_{0} \ldots \ldots \ldots \ldots \ldots \ldots$

3.4 Systematic Uncertainties . . . . . . . . . . . . . . . 32

4 CONFIGURATION FILE AND EVENT RECONSTRUCTION 36

4.1 Configuration File . . . . . . . . . . . . . . . . . 36

4.2 Event Selection . . . . . . . . . . . . . . . . . . . . . . . . . . . . . . . . . . . . . .

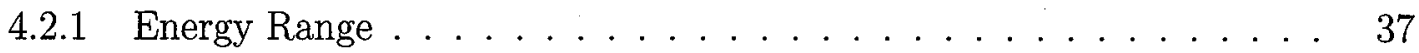

4.2 .2 Invariant Mass Cut . . . . . . . . . . . . . . . 39

5 ANOMALY DETECTION AND QUALITY ASSURANCE 43

5.1 Energy Slope Anomaly . . . . . . . . . . . . . . . . . . . 43

5.2 Strip Anomaly. . . . . . . . . . . . . . . . . . . . . . . . . . . . . . . . . . . . . .

5.2 .1 Events Per Strip . . . . . . . . . . . . . . . . . . . . 45

5.2 .2 Energy to Invariant Mass Correlation . . . . . . . . . . . . . 46

5.2 .3 Invariant Mass Position and RMS Width . . . . . . . . . . . 52

5.3 Bunch Anomaly . . . . . . . . . . . . . . . . 56

6 RUN BY RUN POLARIZATION $\quad 64$

6.1 Average $A_{\mathrm{N}} \ldots \ldots \ldots \ldots \ldots$

$6.2 \sin (\phi)$ fit . . . . . . . . . . . . . . . . . 64 
7 POLARIZATION PROFILE $\quad 69$

7.1 Dedicated Polarization Profile Measurements . . . . . . . . . . . . . . . . 69

7.2 Global Polarization Profile . . . . . . . . . . . . . . . . . . . . 73

7.3 Profile Correction . . . . . . . . . . . . . . . . . . 78

8 JET NORMALIZATION $\quad 81$

8.1 Average polarization over fill . . . . . . . . . . . . . . . 81

8.2 Average Polarization for Jet Normalization . . . . . . . . . . . . . . . . . 84

$9 \quad$ POLARIZATION FOR EXPERIMENTS AND UNCERTAINTIES $\quad 89$

$\begin{array}{lr}\text { A Energy Loss Model } & 95\end{array}$

$\begin{array}{ll}\text { B Bunch Anomaly Examples } & 100\end{array}$ 


\section{Chapter 1}

\section{INTRODUCTION}

The polarization of the proton beams [1,2] at the Relativistic Heavy Ion Collider (RHIC)[3] is measured using both an atomic beam source hydrogen gas jet (H-Jet) [4, 5] and protoncarbon $(\mathrm{pC})$ polarimeters[6,7]. These polarimeters are set up in the 12 o'clock area in the RHIC ring. The H-Jet polarimeter is located at the collision point allowing measurements of both beams. Two identical pC-polarimeters are equipped in the yellow and blue rings, where the rings are separated. The $\mathrm{pC}$-polarimeter measures relative polarization to a few percent statistical accuracy within 20 to 30 seconds using an ultra-thin (typically $6 \sim 8 \mu \mathrm{g} / \mathrm{cm}^{2}$ ) carbon ribbon target, providing fast feedback to beam operations and experiments. The absolute normalization is provided by the hydrogen polarimeter, which measures over $1 \sim 2$ days to obtain $\sim 5 \%$ statistical uncertainty (in Run05). Thus, the operation of the carbon polarimeters was focused on better control of relative stability between one measurement to another measurement rather than measuring the absolute polarization.

The published data of the analyzing power for the elastic polarized proton-carbon scattering is available up to the $21.7 \mathrm{GeV} / c[8]$. There are no published data available at the storage (flat-top) proton beam energy of $100 \mathrm{GeV}$ where the colliding experiment was performed in RHIC. Shown in the Fig. 1.1 is the analyzing power measured by the blue carbon polarimeter during Run04 operation for the extended range of the momentum transfers $-t$. The absolute scale was determined by normalizing the average polarization observed by the carbon polarimeter against the absolute polarization measurements by the hydrogen polarimeter. Nevertheless the precision of the normalization was limited by the statistical accuracy of the jet measurement; $\Delta A_{\mathrm{N}}^{\mathrm{Run} 04} \sim \pm 9 \%$. The strategy is to improve the accuracy year by year with more statistical abundance in the average polarization measurements by the carbon $\overline{P_{\mathrm{pC}}^{\mathrm{Ru}} 05}$ and the hydrogen $\overline{P_{\mathrm{H}-\mathrm{Jet}}^{\mathrm{Ru}} \overline{\mathrm{J}}}$ polarimeters. The improved analyzing power of Run05 $A_{\mathrm{N}}^{\mathrm{Run} 05}$ is given by

$$
A_{\mathrm{N}}^{\mathrm{Run05}}=A_{\mathrm{N}}^{\mathrm{Run04}} \frac{\overline{P_{\mathrm{pC}}^{\mathrm{Run} 05}}}{\overline{P_{\mathrm{H}-\mathrm{Jet}}^{\mathrm{Run} 05}}} .
$$

The curves in the Fig. 1.1 are the model predictions [9] of with and without the spinflip amplitude fitted to the data. The analyzing power for the elastic polarized proton- 
carbon scattering is predicted to be maximized at the momentum transfer of $(-t \sim 0.003$ $\left.(\mathrm{GeV} / \mathrm{c})^{2}\right)$ due to the interference between the electromagnetic and the strong amplitudes (this is known as the Coulomb-Nuclear Interference (CNI) region). In order to take advantage of relatively large sensitivity to the polarization, the recoil carbon atoms were detected near 90 degrees with respect to the beam direction. Kinetic energy range was from 400 to 900 $\mathrm{keV}$, corresponding to a momentum transfer of $0.09<-t<0.23(\mathrm{GeV} / \mathrm{c})^{2}$. The lower the kinetic energy, the larger the analyzing power and the more sensitivity we gain. However, in reality, the present range is constrained by the reliability of the low energy carbon detection as discussed later. Since there is a $t$-dependence in the analyzing power even within the limited $t$ coverage, absolute energy of recoil carbon ion needs to be measured to define the kinematics.

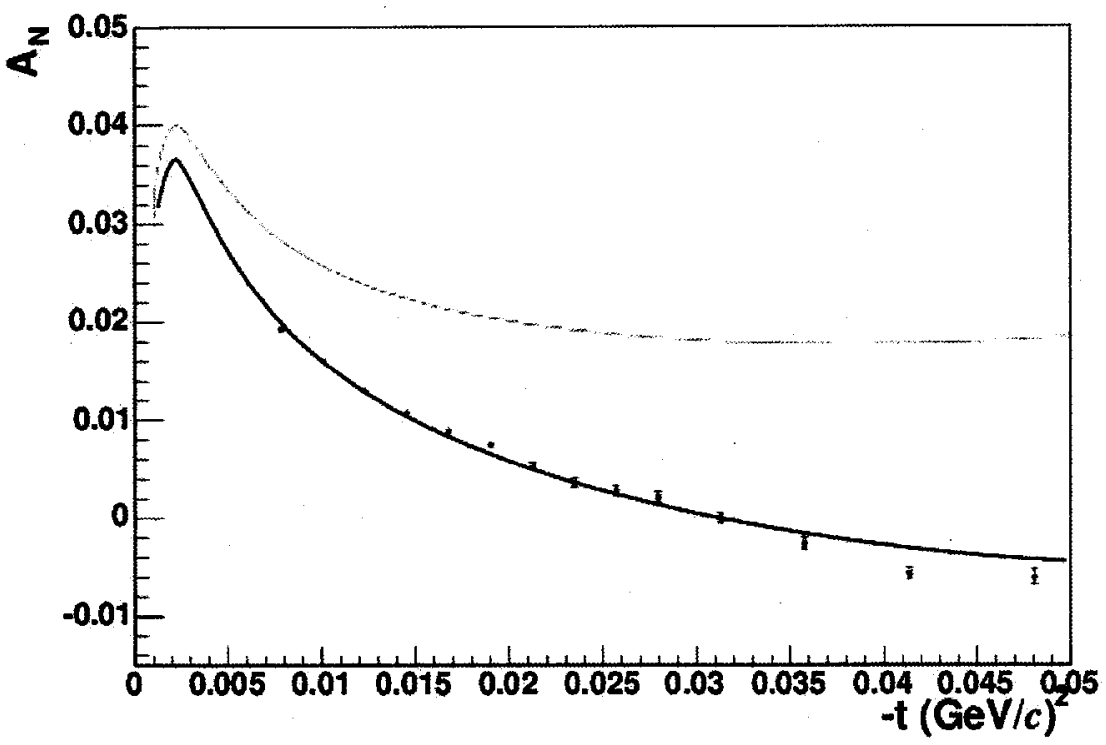

Figure 1.1: The analyzing power measured by the blue carbon polarimeter during Run04 for the extended range of the momentum transfers $-t$. The absolute scale was normalized against the hydrogen polarimeter results in Run04.

Run05 was the first extended operation of polarized proton-proton beams at RHIC. Polarization measurements were executed regularly every $2 \sim 3$ hours while the beams are at storage energy $100 \mathrm{GeV}$ using the carbon polarimeters throughout the three months of running. Continuous efforts were made in the accelerator tuning in order to improve the beam performance, such as polarization, intensity, emittance and backgrounds. Thus these measurements were not necessarily performed always under the same accelerator or detector conditions. The measured data have been carefully analyzed offline in order to estimate any instabilities induced by changed conditions between measurements. Slowly evolving changes, for example, accumulated radiation damage to the detectors is one of the major concerns because of longer exposure to the reactions with higher beam intensity than previous runs. 


\section{Chapter 2}

\section{EXPERIMENTAL APPARATUS}

\subsection{Target System And Detectors}

The carbon polarimeters consisted of a carbon target and six silicon strip detectors. They are all mounted in the vacuum inside a scattering chamber as seen in Fig. 2.1. The photograph shows the scattering chambers of the blue and yellow polarimeters mounted on the blue and yellow ring beam pipes, respectively.

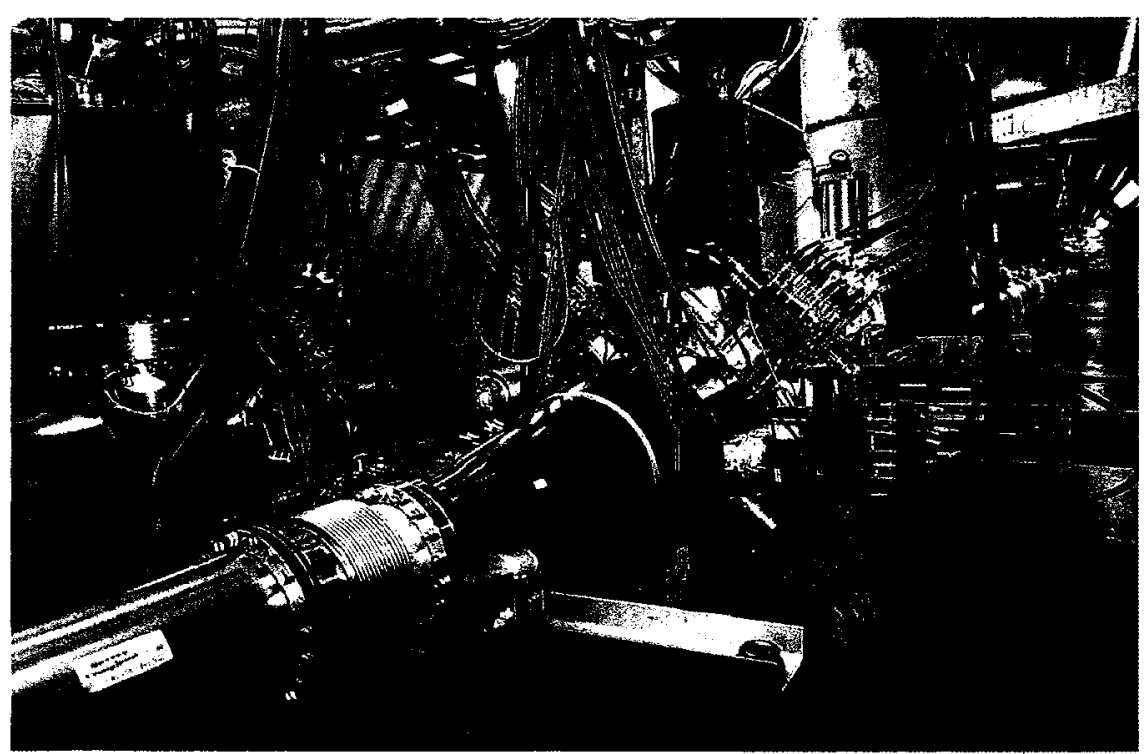

Figure 2.1: The photograph shows the scattering chambers of the blue and yellow polarimeters mounted on the blue and yellow ring beam pipes, respectively.

Very thin carbon ribbon targets have been developed at Indiana University Cyclotron Facility[10]. The targets were made by vacuum evaporation-condensation onto smooth glass substrates. Typically size of $2.5 \mathrm{~cm}$ length with $6 \sim 8 \mu \mathrm{g} / \mathrm{cm}^{2}$ thick and $10 \sim 20 \mu \mathrm{m}$ width 
target was glued both edge on an open side of the "C"-shaped target folder frame as shown in Fig. 2.2. The targets are normally kept away from the beam line and it rotated into the beam only when the polarization measurement is executed, with a choice of 4 vertical and horizontal targets for Run05. The folder with 6 frames in the Fig. 2.2 is the upgraded version of the vertical target folder for Run08. It is crucial to mount multiple targets simultaneously because the target is so thin, and has a certain lifetime against the radiation damage. The target lasted within a week on average during Run05 and the pre-mounted spare target was used without breaking the vacuum to replace the broken one. ${ }^{1}$

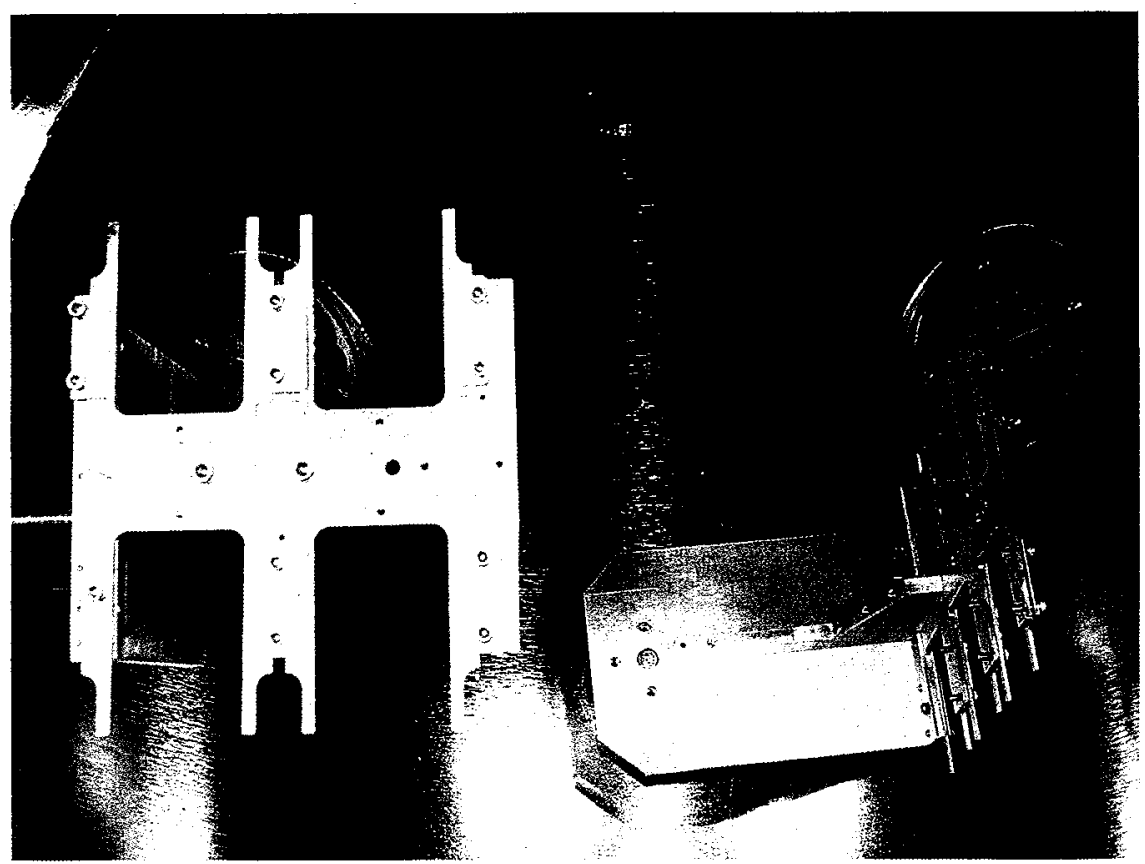

Figure 2.2: The horizontal and vertical target folders viewed from the top of the scattering chamber. The beam goes from the bottom to the top of the picture. (Note this picture is the upgraded one for Run06).

Six silicon sensors were mounted in a vacuum chamber at 45, 90, 135 degrees azimuthally in both left and right sides with respect to the beam with schematic shown in Fig. 2.3. The sensor has $10 \times 24 \mathrm{~mm}^{2}$ total active area, divided into 12 strips of $10 \mathrm{~mm} \times 2 \mathrm{~mm}$ each as shown in the Fig. 2.4. The segmented axis of the detectors are oriented to the azimuthal direction, so there is no segmentation of the detectors in the beam direction. Thus the present setup do not have any sensitivity to the scattering angle of the recoil carbon ions within the acceptance. The thickness of the detector is $400 \mu \mathrm{m}$, fully depleted with the

\footnotetext{
${ }^{1}$ However, still all spare targets were broken in the middle of Run05, and new targets needed to be installed by breaking the vacuum a couple of times during the run. Targets for the yellow polarimeter were replaced on April 6 and May 13 and those of blue one were done on April 19.
} 
operation bias voltage of 100 to $150 \mathrm{~V}$. The strips are made by the Boron implantation $\mathrm{p}$--doping to a depth of $150 \mathrm{~nm}$ on the n-type Si bulk on the side facing the target ${ }^{2}$ The right panel in Fig. 2.5 illustrates the cross section of the silicon sensor. The distance from target to the silicon sensors was $18.5 \mathrm{~cm} .^{3}$

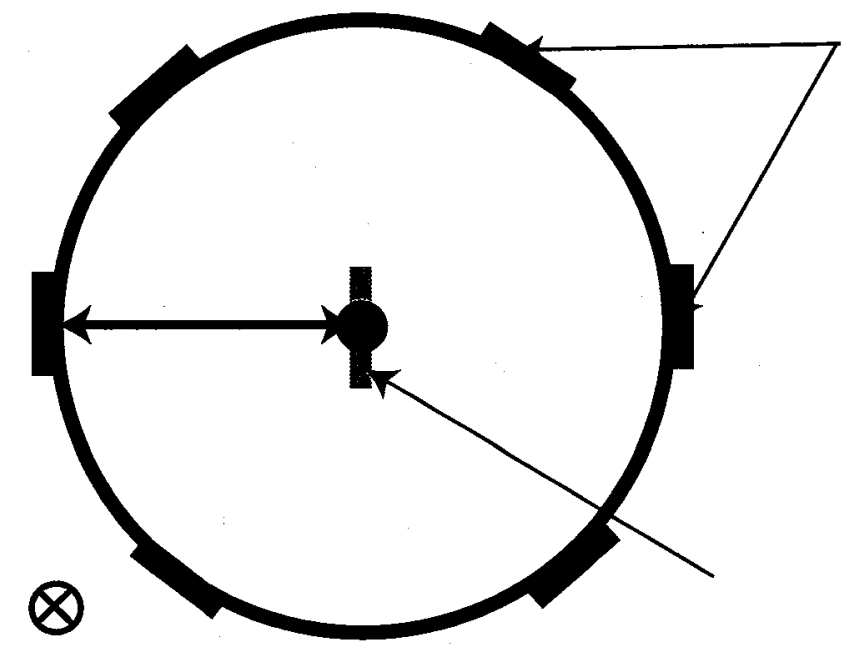

Figure 2.3: The RHIC pC-polarimeter setup. Silicon sensors are aligned 45, 90, and 135 degrees azimuthally in both left and right side with respect to the beam direction. The beam is pointing into the figure perpendicularly.

The horizontal target frame blocks a part of acceptance of the $90^{\circ}$ detectors therefore these $90^{\circ}$ detectors were excluded from the polarization analysis whenever the data were taken with the horizontal targets.

\subsection{Readout Electronics And Data Acquisition}

If the dead time induced by the a read-out system correlated with the polarization sign change, it drives the polarization measurement into uncontrollable false asymmetries. One of the critical part of the proton-Carbon polarimeter is a very fast (dead-timeless) DAQ system. Such system was built based on the fast waveform digitizer[12] (WFD) modules developed at Yale University. Signals from Si strip detectors are preamplified, transferred and shaped to obtain short (40ns FW) pulses with the amplitude proportional to the charge, deposited in

\footnotetext{
${ }^{2}$ The layer to the depth of the Boron implantation is so called dead-layer and corresponding thickness is $150 \mathrm{~nm} \times 2.33 \mathrm{~g} / \mathrm{cm}^{3} \approx 35 \mu \mathrm{g} / \mathrm{cm}^{2}$, which is reasonably consistent with what were initially observed in the both blue and yellow polarimeters at the beginning of Run05 as presented in Fig. 3.11

${ }^{3}$ From Run05 on, a vacuum flange spacer was mounted for each detector feedthrough which effectively changed the distance between target and silicon detectros from $15 \mathrm{~cm}$ to $18.5 \mathrm{~cm}$.
} 

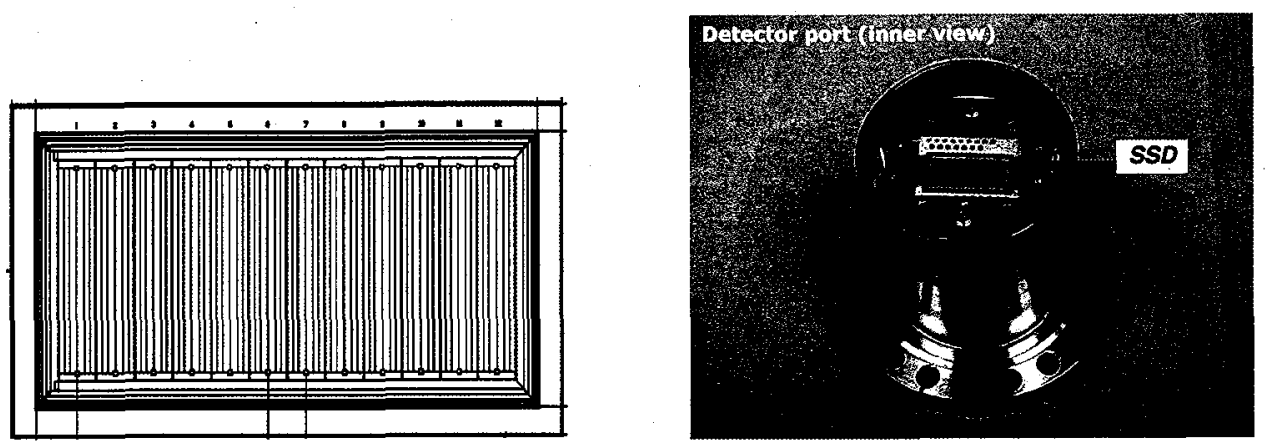

Figure 2.4: (Left) the mechanical drawing of the silicon sensor. Each sensor is segmented into 12 strips with $2 \mathrm{~mm}$ pitch. (Right) The silicon detector mounted on a support structure attached on a flange.

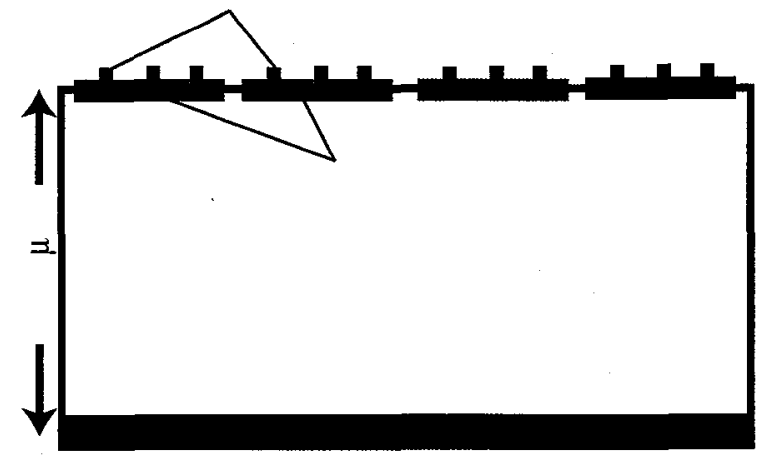

Figure 2.5: The cross section of the silicon sensor. 
the detector. The pulse shapes are then digitized at the equivalent frequency of $420 \mathrm{MHz}$ and analyzed inside the WFD modules, providing the recoil carbon deposited energy and timeof-flight. The events are then filtered through look up tables (LUT), checking the kinematic correspondence between the obtained values for carbon identification. The selected events are used to increment scalers and histograms in the modules, and can be stored in the onboard memory. Shown in Fig. 2.6 is the typical kinematic correlation plot identifies elastic carbon events and backgrounds. The vertical and horizontal axes represent the time-of-flight and the kinetic energy reconstructed based on the time offset $t_{0}$ and an effective dead-layer thickness $x_{\mathrm{dl}}$ table used in online. The details of $t_{0}$ and $x_{\mathrm{dl}}$ are discussed in section 3.2. The event selection was applied in relatively loose manner than that of the offline analysis, i.e. typically \pm 15 ns in time-of-flight and the energy of $360<E<1100 \mathrm{keV}$ as shown in the solid curves in the figure. The comparison of the constant time-of-flight cut and the invariant mass cut is presented in Fig. 2.7. As can be seen $\pm 15 n s$ constant time-of-flight cut is looser than typical $3 \sigma$ mass width cut throughout the range of $400<E<900 \mathrm{keV}$, which was used for the Run05 offline analysis.

The primary difference in the event selection between online and offline are summarized below:

- Configuration file which contains $x_{\mathrm{dl}}$ and $t_{0}$ for every strips. The online configuration files are not as frequently updated as offline. When the fractuation of the $x_{\mathrm{dl}}$ is pronounced, this difference drive visible difference in resulting polarizations between online and offline.

- Energy range.

- Kinematics reconstruction formula. For reducing cpu time, online employs linear formula instead of 5th order polinomials presented in Appendix. A which was used for offline analysis and the effective dead-layer fit.

- Disabled fill and strips. Some suspiciously behaving strip and fills are disabled in offline analysis. In online there is no masking feature.

The main advantage of such an approach is that there is no data transfer to the host computer during a data taking run, which makes the system really dead-timeless. The analyzing part is capable of processing of one event per RHIC bunch crossing period (110ns) resulting in maximum of $\approx 10^{7} / s$ events per channel.

The WFD is a CAMAC module hosting 4 independent channels as shown in Fig. 2.8 with common storage SDRAM (64 MByte) and CAMAC control circuity. In each channel the input signal is split into three, two of which are delayed $1 / 3$ and $2 / 3$ of the ADC digitization period. Three 8-bit ADCs synchronously start conversions at $140 \mathrm{MHz}$ resulting in triple equivalent digitization frequency. All the waveform analysis is done inside the Vertex- $E$ Xilinx FPGA chip[13] at $70 \mathrm{MHz}$ clock frequency. The analysis algorithm is rather specific since it has to process every 6 waveform points in parallel (the FPGA clock is only $1 / 6$ of the digitization frequency). 


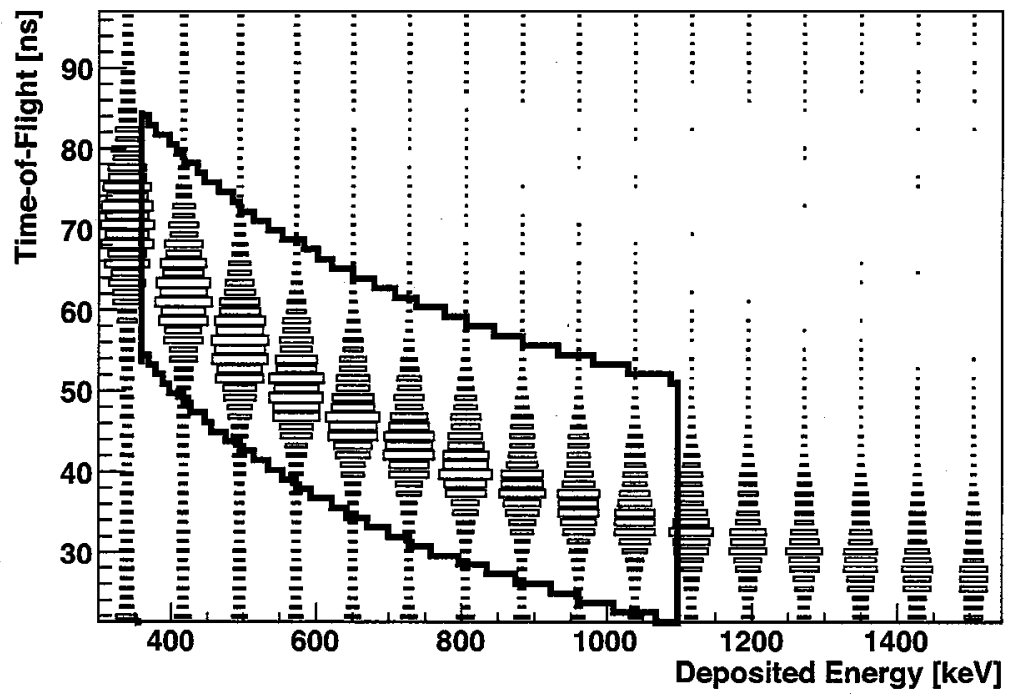

Figure 2.6: The typical kinematic correlation plot observed in the online scaler mode. The vertical and horizontal axes represent time-of-flight and the kinetic energy. Surrounded by the red curves are selected events as the elastic recoil carbon events in online.

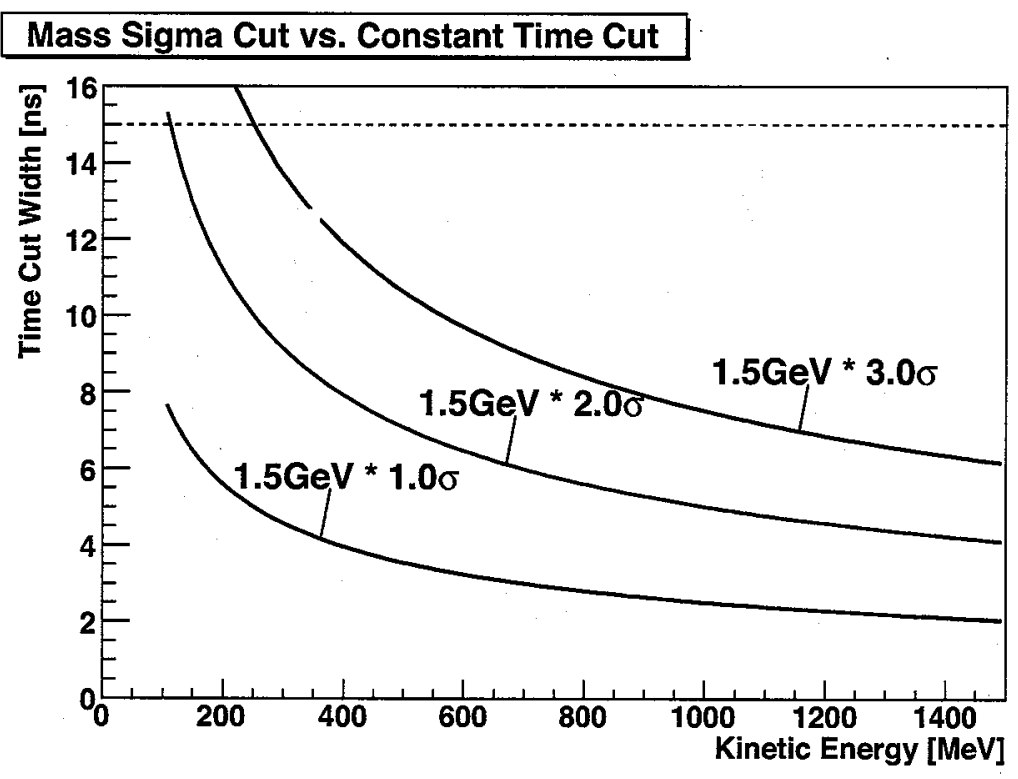

Figure 2.7: The comparison between constant ToF cut and the invariant mass width cut. 3 curves are $3 \sigma$ boundary for the typical invariant mass width of $1.5 \mathrm{GeV}$ plotted as a function of the kinetic energy of a carbon ion. 


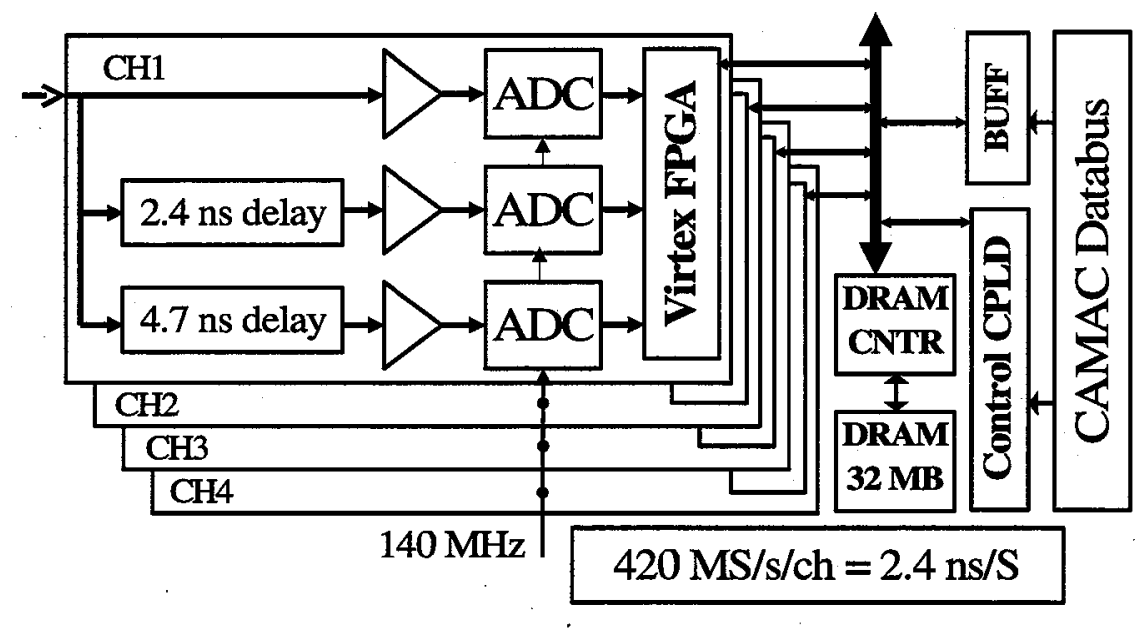

Figure 2.8: A block diagram of the wave form digitizer.

The block diagram of the analyzing circuits in the FPGA is shown in Fig. 2.9. The input signal passes through a digital filter for noise reduction and partial compensation for different amplifications of delayed sub-channels. A level trigger is used to determine the presence of a significant signal in a particular bunch crossing period, and if the signal is not detected, the ADC values are used for the baseline calculations. The baseline is determined individually for all three sub-channels to compensate for different amplifier offsets and is averaged over the 16 latest bunch crossing periods with no significant signal. The baseline is then subtracted and the signal is stored in FIFO, from which it can be directly read out as a waveform or taken for further analysis.

The analysis is of the conveyor type and takes up to 5 stages, each stage corresponding to a sequential bunch crossing. On the first stage the whole waveform is used to define the signal amplitude (maximum), integral and time at maximum. The second stage implements $1 / 4$ constant fraction discriminator (CFD) based on the amplitude value defined at the first stage. The CFD time and amplitude is then used to filter the event through a LUT, which is preprogrammed to account for energy-TOF correlation, specific for carbon ions. Another LUT is used to compare the amplitude and integral values, which deviate from proportionality in case of two particles arriving within one bunch crossing. If both LUTs report positive result, all signal parameters are stored in the result FIFO and used to fill the on-board histograms. The FPGA keeps track of the bunch and revolution numbers, as well as of the bunch polarization pattern, which allows a variety of histograms including distributions of the energy (polarization sorted) and bunch number and 2D time-amplitude histograms. Their contents can be read out after the data taking and is sufficient for beam polarization determination. Yet for better understanding and debugging purposes the signal parameters from the result FIFO can be transferred to the on-board storage memory, limiting the maximum event rate to $3 \cdot 10^{6} \mathrm{~s}^{-1}$ per channel. 


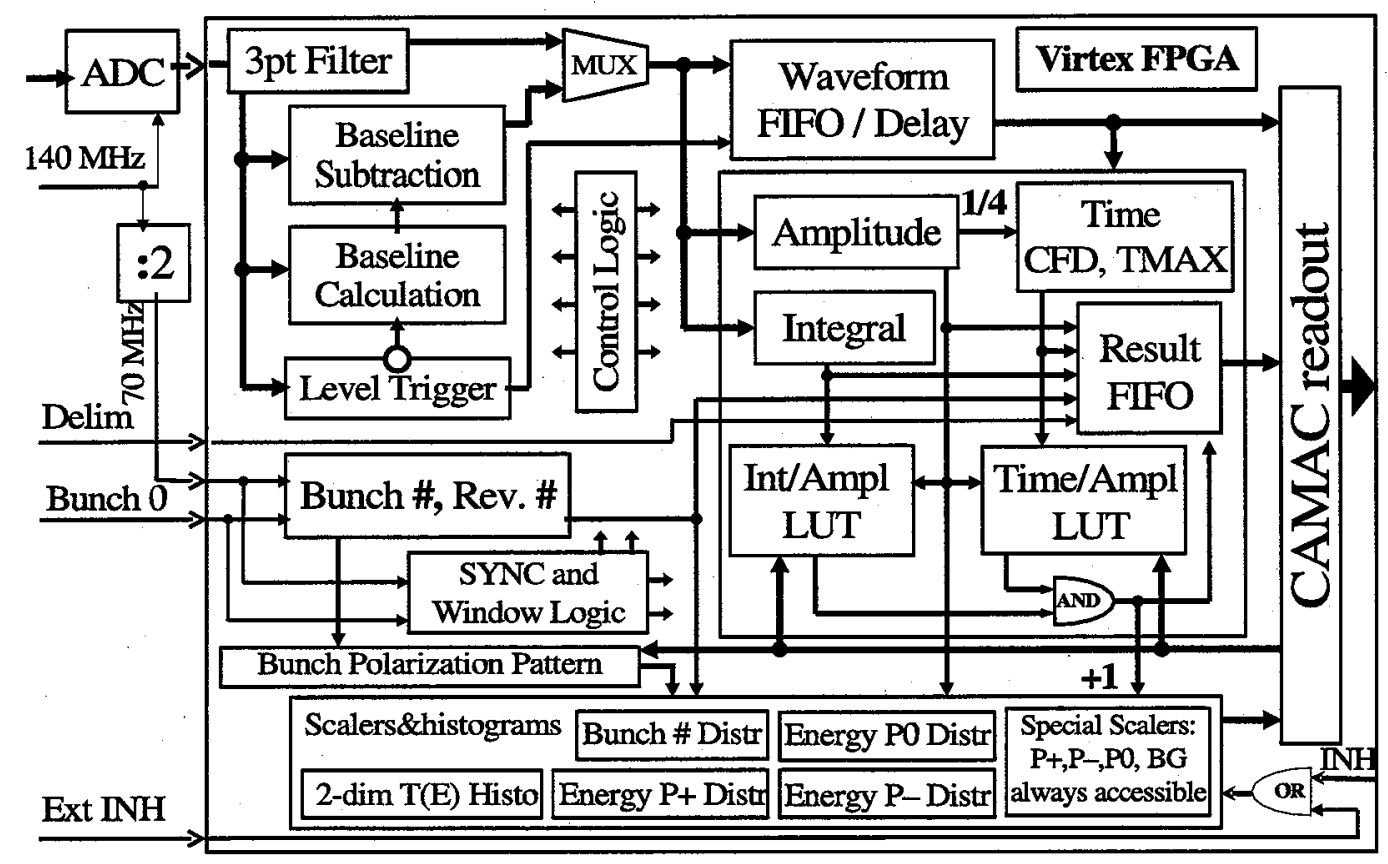

Figure 2.9: A block diagram of the wave form digitizer per channel. 


\section{Chapter 3}

\section{CALIBRATION AND ENERGY CORRECTION}

\subsection{Energy Calibration}

The energy calibration of the silicon detectors is performed using an $\alpha$ source (241Am, $(85 \%)$, $5.443 \mathrm{MeV}(12 \%))$. The sources were mounted on the detector franges and shine detectors on the opposite side of the beam. Dedicated calibration runs were executed several times throughout Run05 and typically very minor gain changes were observed for all the strips. Shown in Fig.3.1 is a typical ADC distribution of the calibration for a single strip. The gain factor was determined from the mean of the Gaussian fit to the peak of $\alpha$ events. Since the kinetic energy of the $\alpha$ is more than factor of 5 larger than the maximum Carbon energy we use for the normal polarization measurements, the signal output for the calibration was attenuated by 5 in order to accommodate within the dynamic range of ADC. Thus the gain factor is also scaled by 5 for use. About $1000 \sim 1200$ events are accumulated per strip. The resulting precision to determine the peak position is less than $0.1 \%$, which is negligibly small compared to other uncertainties.

The acceptance opened up by the shields were somewhat slightly smaller than the actual detector acceptance. As a consequence, a couple of strips located at the edge of the detectors (away from 90 degrees) barely accumulated alpha particles from the source and showed difficulty in evaluating relevant gain factors from the data. Since we observed consistent gain factors each other for the strips which were free from this problem and were quite stable throughout the Run05, we calculated the average gain factor from these "good" strips and assigned it for the problematic strips.

The systematic uncertainty of the gain is studied by calculating the dependency of the polarization on the gain. The gain is artificially changed within the range of $\pm 10 \%$ using the typical data one from yellow (7279.106) and another from blue (7279.005) measurements, respectively. The resulting dependencies are plotted in the Fig. 3.3. As shown in the figure, the dependences appeared $-6.0 \sim-6.1 \%$ per $10 \%$ scale factor in the absolute polarization scale. 


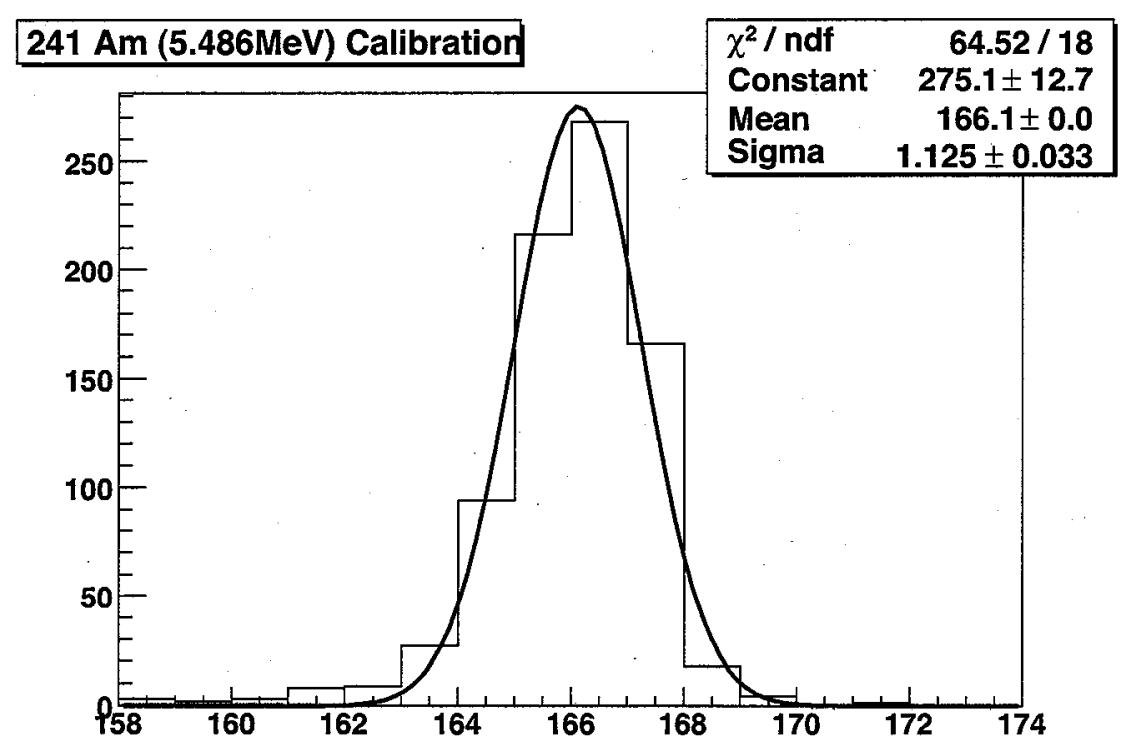

Figure 3.1: A typical example of gain factor determination using Am source peak. A curve is the Gaussian fit to the observed energy peak of the $\alpha$ particle.

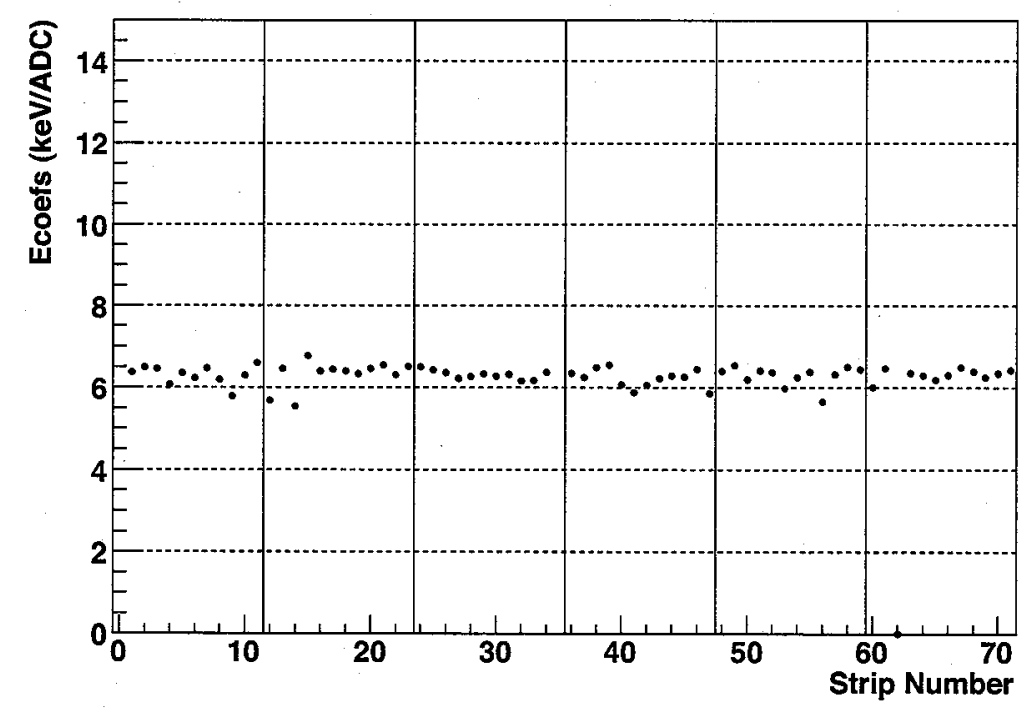

Figure 3.2: Gain factors for all strips of the blue polarimeter determined using ${ }^{241} \mathrm{Am}$ source. 


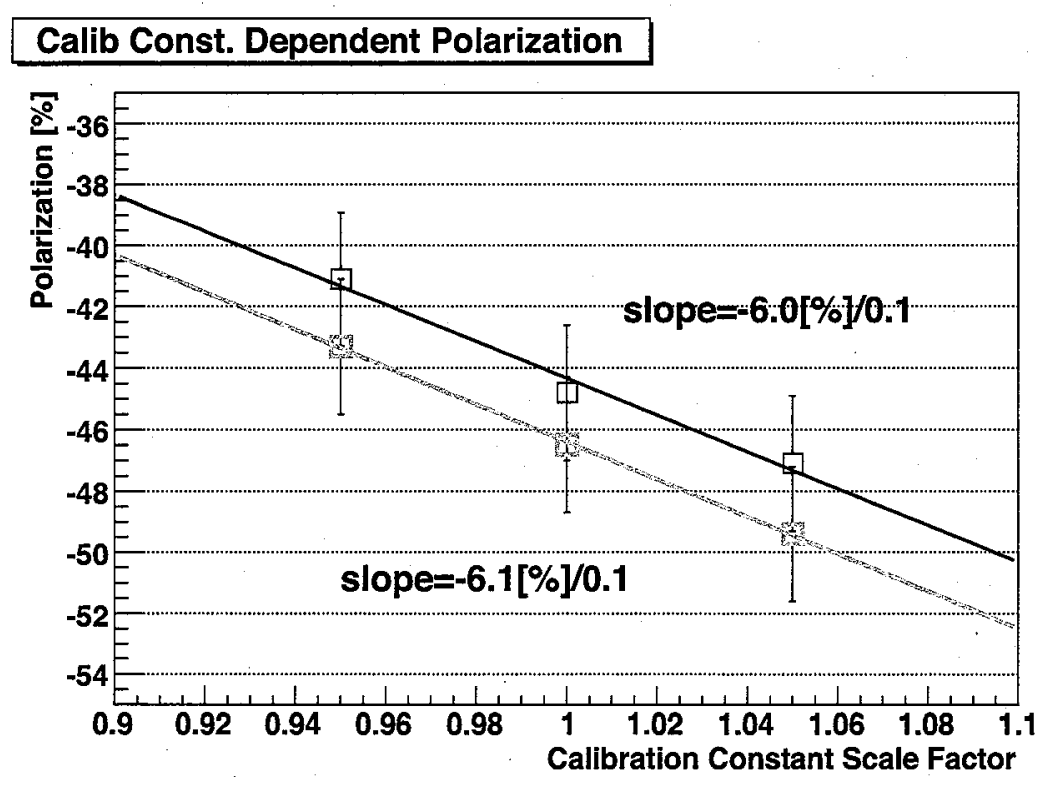

Figure 3.3: Polarization dependence on the gain factor.

The artificial tweak of the energy scale will somewhat shift the invariant mass position from where the carbon mass supposed to be. Normally we require the carbon mass to be reconstructed to be at the right position for the all strips by optimizing the magnitude of the energy loss in the effective dead-layer and the timing offset which will be discussed in details in the next section. As a consequence, the impact of the artificially tweaked energy scale is somewhat relieved as compared in Fig. 3.4. The reason for the smaller dependence comes from the compensation of the gain factor in the dead-layer estimation The artificially imposed additional (reduced) gain factor should be interpreted as thinner (thicker) effective dead-layer in order to make sense of the kinematic reconstruction. To be concrete, the change in the energy scale from 1.0 to 1.1 , the effective dead-layer decreased by about $7 \mu \mathrm{g} / \mathrm{cm}^{2}$. Thus the effect is somewhat absorbed by the free parameters $\left(x_{\mathrm{dl}}, t_{0}\right)$ in the effective deadlayer fitting process and results in small impact on the polarization.

\subsection{Effective Dead-Layer and Energy Correction}

The $\alpha$ calibration, however, does not effectively probe the surface region of the detectors where the sub-MeV carbon ions stop. These low energy carbon ions entering the silicon detector penetrate the p+ doping layer (dead-layer) first, and then enter the sensitive part of the detector, stopping at a depth of $<1.3 \mu \mathrm{m}$. The energy loss of the carbon in the dead-layer is energy dependent and can be described by a known function of energy[11]. This energy loss is a significant fraction of the carbon energy, for example $30 \%$ for $400 \mathrm{keV}$ carbon with a dead-layer thickness of $50 \mu \mathrm{g} / \mathrm{cm}^{2}$ as shown in Fig.3.5. In addition to this energy loss, 

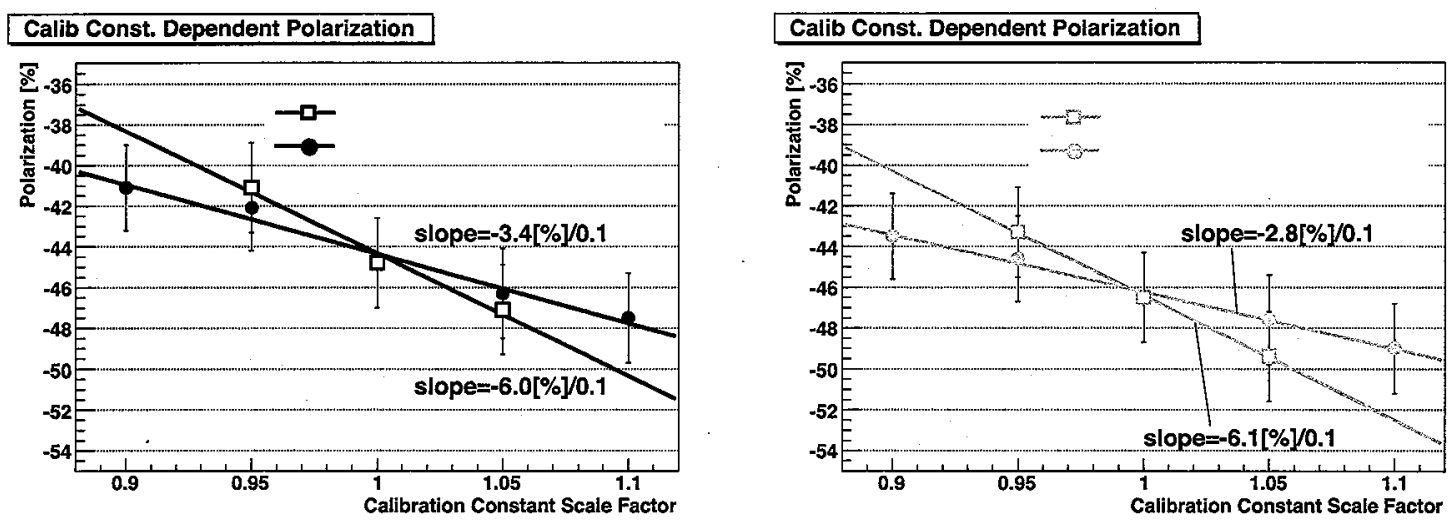

Figure 3.4: Polarization dependence on the gain factor for the blue (left) and yellow (right) polarimeter measurements. Open squares and solid circles represent without/with the deadlayer fitting compensation.

the charge collection near the surface dead-layer can be affected by radiation damage. We have been unable to separate whether a change in the detector response either comes from a changing charge collection or from energy-dependent energy loss in the dead-layer. We consider the net energy correction, due to both the dead-layer and reduced charge collection near the surface, as a varying dead-layer thickness with the energy-dependent energy loss. As illustrated in Fig. 3.6, a realistic smooth transition of the efficiency between the dead-layer and fully efficient charge collection region (left) was treated as discrete transition (right) introducing "effective" dead-layer $x_{\mathrm{dl}}$. This effective dead-layer thickness can be determined through a fit to the kinematical correlation of measured time-of-flight and energy correlation for carbon recoils.
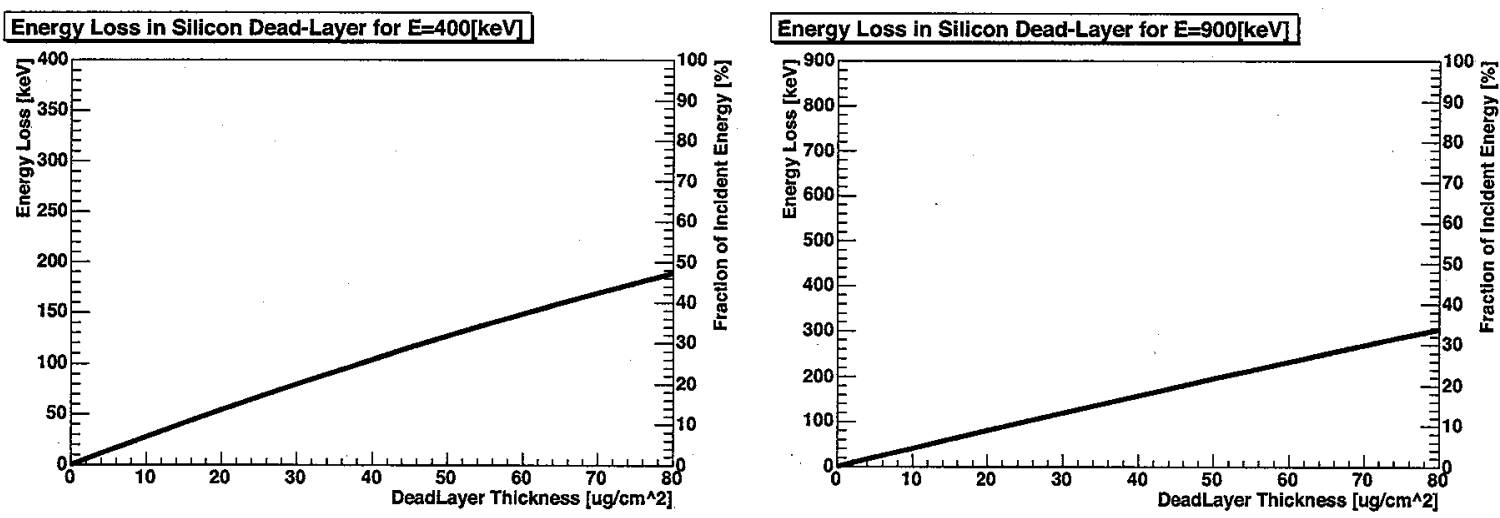

Figure 3.5: The fraction of the energy loss in the dead-layer of silicon sensor for the carbon ion with its kinetic energy of $400 \mathrm{keV}$ (left) and $900 \mathrm{keV}$ (right). 

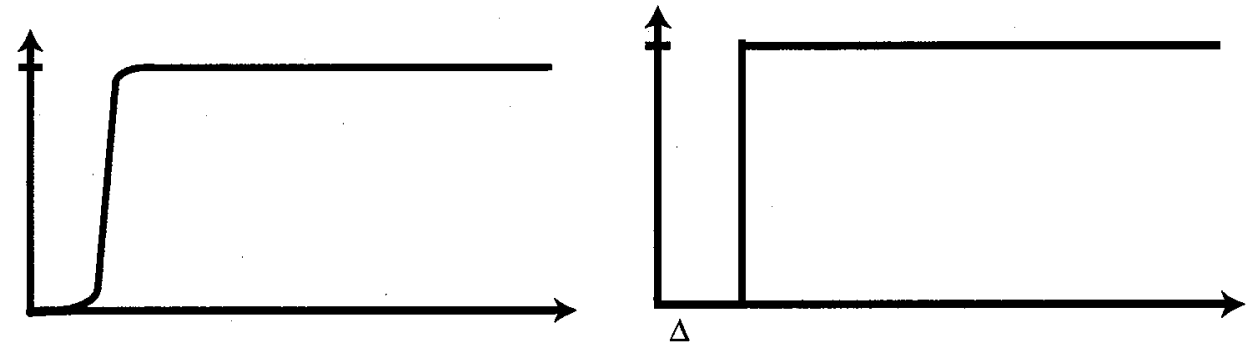

Figure 3.6: (left) An image of realistic charge collection efficiency as a function of depth of the silicon detector. (right) The concept of the effective dead-layer. Truncates the smooth transition between the dead-layer and the fully depleted region by the discrete connection.

Shown in Fig.3.7 is an example of the fit using the non-relativistic kinematic formula,

$$
E_{\text {meas }}+E_{\text {loss }}\left(x_{\mathrm{dl}}, E\right)=\frac{1}{2} \cdot M \cdot \frac{L^{2}}{\left(t_{\text {meas }}+t_{0}\right)^{2}}
$$

where $M, L, t_{\text {meas }}$ and $E_{\text {meas }}$ are the mass, the flight path length, the measured time-offlight, and the energy of the recoil carbon ion, respectively. A time offset $t_{0}$ and the effective thickness of the dead-layer $x_{\mathrm{dl}}$ were set as free parameters. The energy correction $E_{\text {loss }}$ has dependency on the $x_{\mathrm{dl}}$ and the incident energy of the Carbon ions $E$. The time-of-flight $t_{\mathrm{tof}}$ is given as $t_{\text {tof }}=t_{\text {meas }}+t_{0}$. Details of the parameterization of $E_{\text {loss }}\left(x_{\mathrm{dl}}, E\right)$ in the silicon surface are discussed in Appendix I. The empirical fit was applied to all polarization measurements during physics stores and evaluated the effective dead-layer thickness for each measurement independently to trace the stability.

Shown in Fig.3.7 is a typical dead-layer fit for a given strip. The data is from typical polarization measurement, but the events are spursified by factor of 10 just for the display purpose. Horizontal axis shows the observed ADC multiplied by the gain factor (unit is in [keV], whereas the vertical axis is the observed TDC in the unit of [ns] (not corrected for $t_{0}$ ). The curve with finite band in the middle of the plot represents for Carbon ion events. Small, but there are some $\alpha$ backgrounds come from inelastic reaction distribute around small time-of-flight and measured energy region. Relativistic backgrounds such as prompt photons and pions, etc should appear around the time-of-flight of zero region, but they are tuned to be out of time window gate otherwise they dominate the DAQ live-time.

The typical RHIC beam bunch is longitudinally spread by a few [ns] in $\sigma$. Since this is a single arm detection, we do not have better absolute timing measurement than this longitudinal spread. The horizontal/vertical width of the Carbon ion band in the Fig.3.7 is determined by the longitudinal spread of the beam bunch and the energy resolution of the detection system.

Every dead-layer fit is performed using the Equation, assuming the event entries are all Carbon ions. Any background entries of $\alpha$ particles in the scatter plot drives the fitting to 


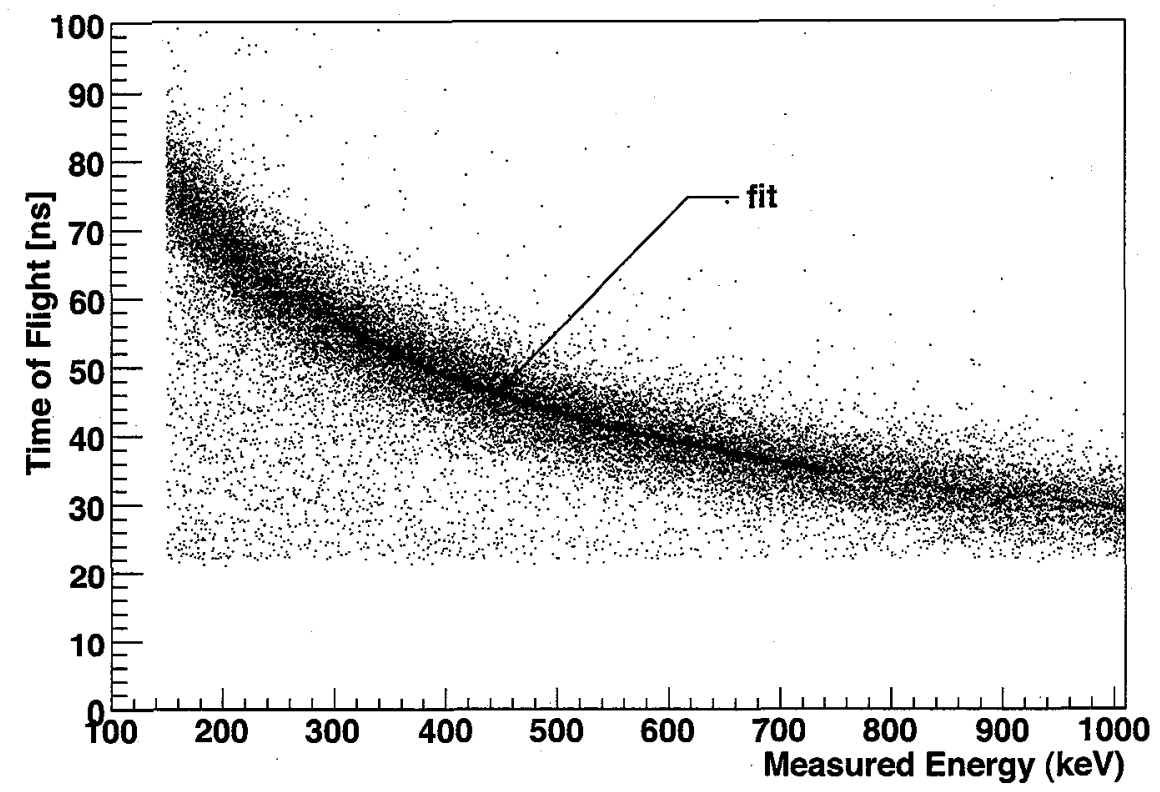

Figure 3.7: An example of the kinematical fit as the energy correction (dead-layer thickness) and time offset $t_{0}$ as free parameters. Fitting range was calculated assuming dead-layer of $40 \mu \mathrm{g} / \mathrm{cm}^{2}$ from standard kinetic energy range $400 \mathrm{keV}-900 \mathrm{keV}$.

loose the precision. As can be seen in Fig.3.7, there are some backgrounds contaminated in the kinematic fit. Since the typical background level is negligibly small though, there are some cases the bulk of $\alpha$ events in $t_{\text {tof }} \rightarrow 0$ and $E_{\text {meas }} \rightarrow 0$ corner pulls the fitting function to undershoot the Carbon band, which results in overestimating the effective deadlayer thickness. In order to eliminate these background contaminations to the dead-layer fit a particle identification cut was applied on the reconstructed invariant mass as shown in Fig.3.8. Of cause the kinematic reconstruction requires the knowledge of the effective deadlayer thickness, which is the unknown free parameter of the dead-layer. A event selection by the kinetic energy $E$ was also made, since there are slight kinetic energy range dependence on the dead-layer fit as shown in Fig.3.9. The energy range study was made for randomly selected 50 runs per blue and yellow polarization measurements, respectively. Shown in the figure is the average effective dead-layer thickness of these 50 runs and all active strips of each run.

Thus the energy range $400 \leq E \leq 900 \mathrm{keV}$ was selected as inputs to the dead-layer fit. The range is consistent with the event selection made for the polarization calculation and was always used for all Run05 measurement. Here again, the energy range relies on the reconstructed kinetic energy which requires the knowledge of the dead-layer thickness. This dilemma was resolved by applying the dead-layer fits in an iterative manner. The energy range was calculated for the first iteration without the invariant mass cut based on the effective dead-layer thickness of $50\left[\mu \mathrm{g} / \mathrm{cm}^{2}\right]$ as a typical thickness. For the second and later 


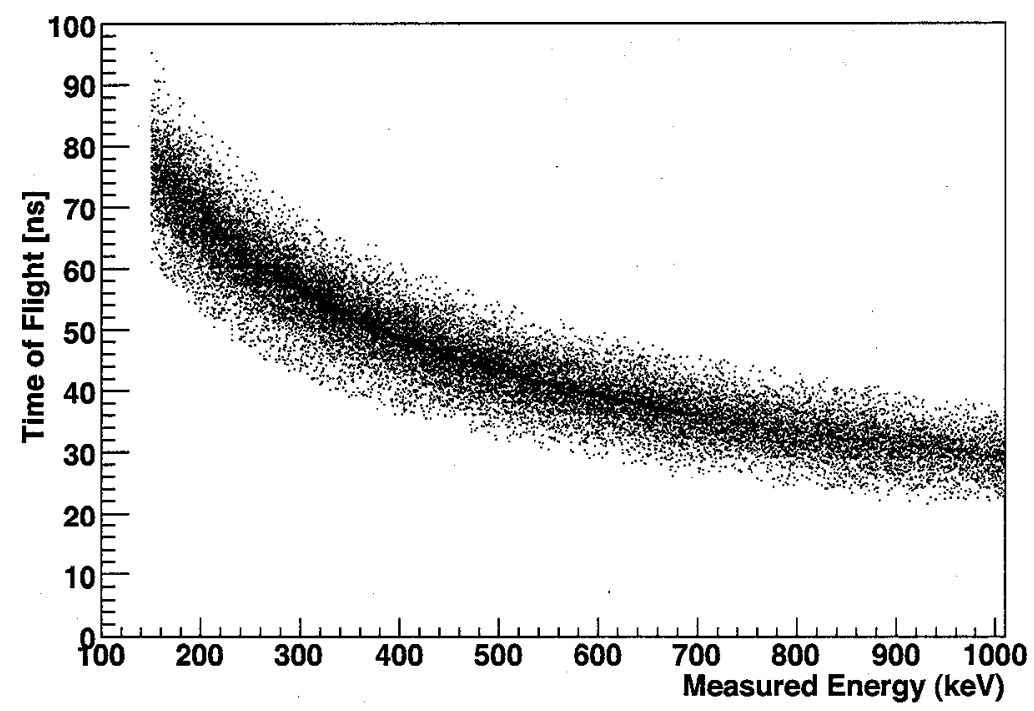

Figure 3.8: Kinematical fit on data which are applied $3 \sigma$ cut on the reconstructed invariant mass of Carbon.
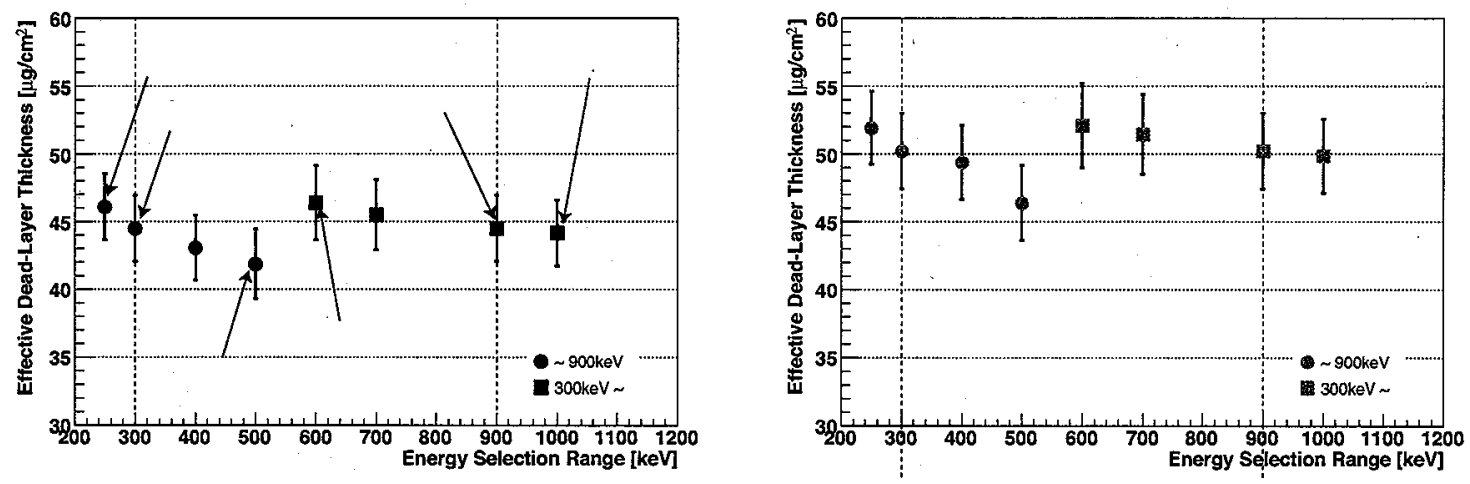

Figure 3.9: The dependence of the effective dead-layer thickness on the kinetic energy range as inputs of the deed-layer fit. Left panel shows the dependency study for blue and right one shows yellow polarimeter measurements. Solid circle data points are plotted at the minimum cut off energy on the horizontal axis while solid square data points are plotted at the maximum cut off energy. The maximum and minimum cut off energies for these data points are 900 and $300 \mathrm{keV}$, respectively. 
iterations, the energy correction was calculated based on the best fit results of the previous iteration. The convergence for $\overline{t_{0}}$ and $\overline{x_{\mathrm{dl}}}\left(\overline{t_{0}}\right.$ and $\overline{x_{\mathrm{dl}}}$ are averages of $t_{0}$ and $x_{\mathrm{dl}}$ for all active strips) below $1 \%$ compared to the previous iteration results are obtained within a couple iterations.

Shown in the Fig. 3.10 are the result of the dead-layer fit for all strips. The top figure shows the effective dead-layer as a function of the strip identification number whereas the bottom figure shows $t_{0}$ distribution. The group of 12 strips which are separated by the vertical lines belong to the same detector. Some data points appear in zero for both $x_{\mathrm{dl}}$ and $t_{0}$ are dead strips. Sold horizontal lines in the top figure represent the weighted average of a given detector. For Run05 analysis, we employed this detector average for the energy correction rather than the individual strip result for following two major reasons: 1) the real dead-layer, i.e. a surface coating plus the doping region, supposed to be common to all strips in the detector. Although this is not necessarily the case for the present effective dead-layer definition which includes any inefficiencies of electronics, the obtained effective dead-layer thicknesses are still dominated by the thickness expected by the doping depth. 2) The risk to employ unreasonable dead-layer fitting results is reduced by taking average of 12 strips weighted by the parameter error multiplied by the $\left(\chi^{2}\right)^{2}$. The $\left(\chi^{2}\right)^{2}$ weight was employed empirically just to be free from bad fit in a negligible level and no mathematical meaning is required from it. The error bars in the top panel of Fig. 3.10 shows the fitting error multiplied by the $\left(\chi^{2}\right)^{2}$, whereas error bars bottom panel represent the fitting error only.

To be consistent with the averaged dead-layer $\overline{x_{\mathrm{dl}}}$, the $t_{0}$ for every strips were calculated again through the dead-layer fit with $\overline{x_{\mathrm{d} l}}$ as a fixed parameter and $t_{0}$ as an only parameter after the every iterations. Blue and red squares in the bottom plot in Fig.3.10 represent the results of two parameters $\left(x_{\mathrm{dl}}\right.$ and $\left.t_{0}\right)$ and one parameter $\left(t_{0}\right)$ fits.

The oscillation in $t_{0}$ between odd and even strip identification numbers come from different cable length among them. This relative timing between strips supposed to be constant unless there are any timing tunes in the electronics during Run05 operation. This is confirmed as an one of the systematic checks of the dead-layer fit.

\subsection{Stability And Dependency}

Shown in Fig.3.11 are the estimated effective dead-layer thickness in units of $\mu \mathrm{g} / \mathrm{cm}^{2}$ averaged over all active strips (excludes strip identification number 12 and 63, which were dead throughout Run05). Each data point represents one measurement performed at the beam energy of $100 \mathrm{GeV}$ (solid circle) or $24 \mathrm{GeV}$ (open circle) in blue (left) and yellow (right) rings. Data points are plotted only for measurements performed during the physics stores. They are plotted as a function of the fill number. As can be seen from the figure, the resulting effective thicknesses of dead-layers are not constant and change from measurement to measurement.

One pronounced discrepancy is the data taken between the injection and the store energies. The data points at the injection energy are systematically thinner than the those of 

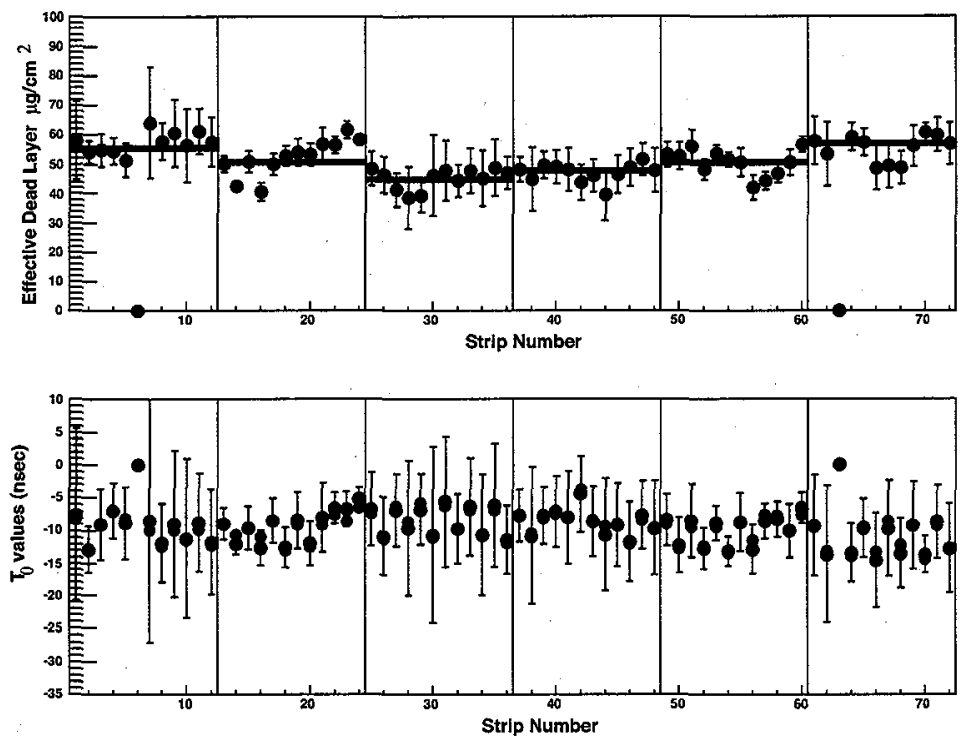

Figure 3.10: Effective dead-layers (top) and $t_{0}$ (bottom) for each strips in the blue polarimeter. Solid lines in the top panel represents the weighted average per detector. The data points at the effective dead-layer thickness of zero and $t_{0}=0$ are dead strips. The blue and red circles in the bottom plots represent results of one- and two-parameter fits.
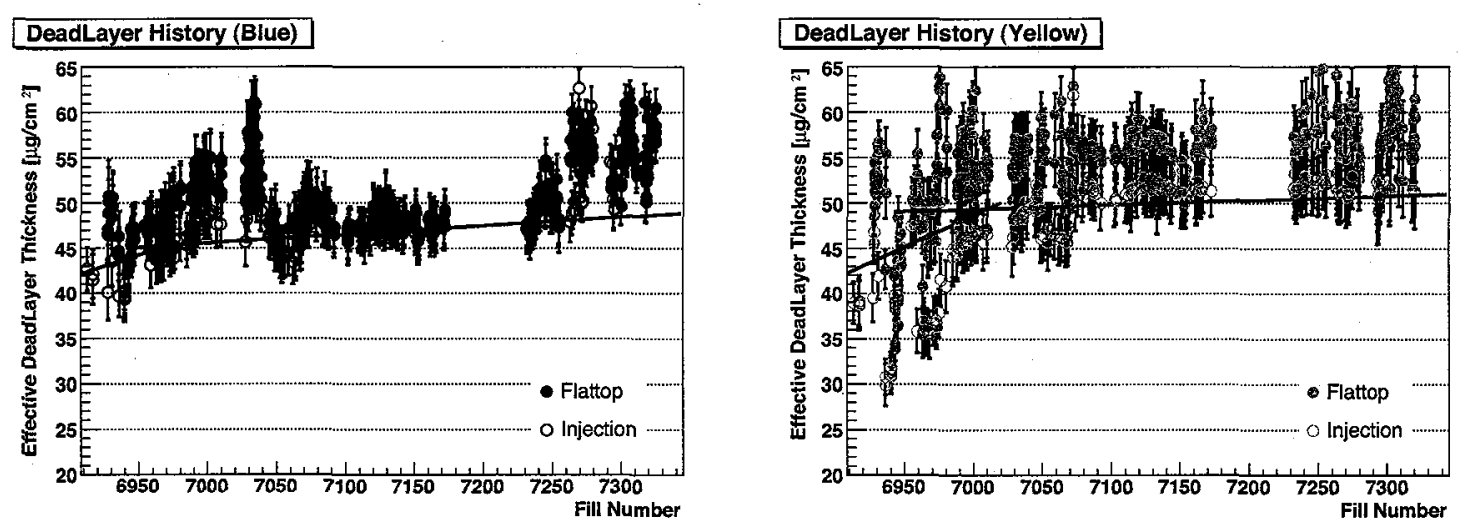

Figure 3.11: Stability of the effective dead-layer thickness averaged over all active strips plotted as a function of fill number for blue (left) and yellow (right) polarimeters, respectively. Solid and open circles are the data taken at the flat-top and the injection energies, respectively. Solid line guides the gradual increase of the effective dead-layer thickness as a function of time (accumulated radiation). 
the store energy. These problems are studied in details in this section. For the purpose of a clarification, major points of the trends and problems found in these figures are itemized below:

1. Increasing $x_{\mathrm{dl}}$ as a function of fill number in general.

2. The increasing trends change slopes around the fill 7000 .

3. $x_{\mathrm{d} 2}$ fluctuates locally.

4. $x_{\mathrm{dl}}$ are in general thinner for the data taken at the injection energy than those of at the store energy.

In order to investigate the dependence of the effective dead-layer thickness on other experimental conditions, correlations to the following quantities are studied: 1) event rate 2) beam intensity per bunch. The event rate is not necessarily proportional to the beam intensity because it depends on the target condition, e.g. target transverse position with respect to the beam intensity profile, target thickness, etc. Thus they are treated separately here.

\subsubsection{Peak Beam Intensity Dependence}

Shown in Fig.3.12 are the intensity dependency of the effective dead-layer. As seen from the Fig. 3.13, the beam intensities are not necessarily uniform. For this particular beam intensity dependence study, any events belongs to the bunches with the intensity beyond \pm $5 \%$ of the average intensity were excluded from the effective dead-layer determination. Each data point in Fig.3.12 are plotted for the average on the horizontal axis.
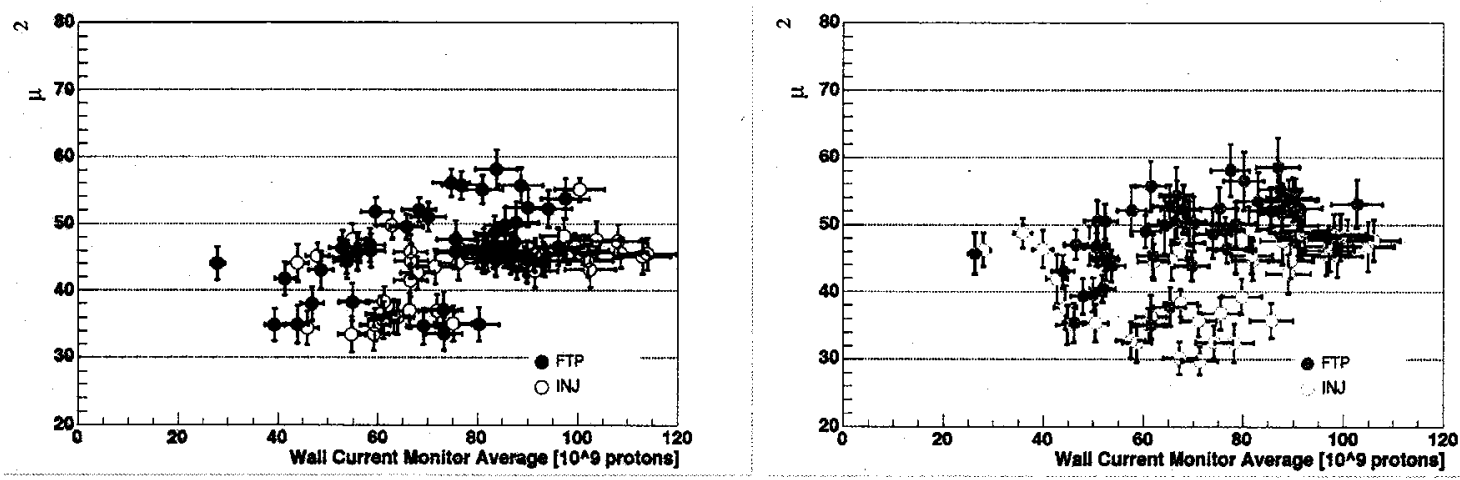

Figure 3.12: The effective dead-layer dependence on the beam intensity per bunch for blue (left) and yellow (right) polarimeters, respectively. 


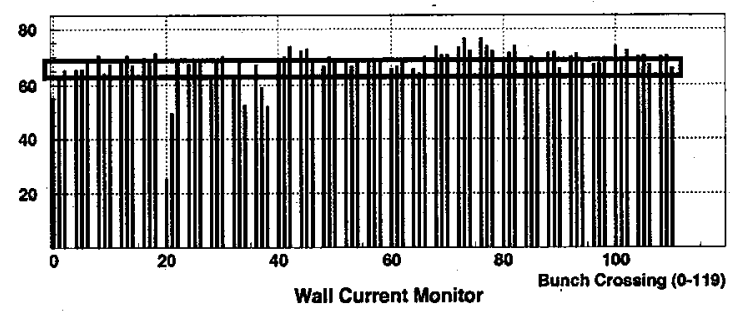

Figure 3.13: A sample of the beam intensities per bunch measured by the wall current monitor[16]. Bunches whose WCM intensity within $\pm 5 \%$ from the average were selected for the intensity study.

In contrast to the event rate dependence case which is discussed below, no obvious correlations were observed. From this fact, we can safely conclude that either 1) the measurement, at least the dead-layer determination, is not sensitive to the beam halo driven backgrounds (even if they exist) or 2) the beam halo driven backgrounds are negligibly small in the present measurement.

\subsubsection{Average Event Rate Dependence}

The event rate studied here is defined by the total number of carbon events (all active strips are combined) within the energy and the $3 \sigma$ invariant mass event selection divided by the duration of the measurement. This is the average event rate in contrast to the instantaneous peak beam intensity discussed in the previous section. Shown in Fig.3.14 are the correlation plots of the effective dead-layer and the average event rate for the blue (left) and yellow (right) polarimeters. There are clear trend of the positive slope, i.e. the higher the rate, the thicker the effective dead-layer becomes, in both blue and yellow cases. However, the interpretation of this correlation is not too straightforward, because the radiation damage effects can also contribute to the slope in the same sign, i.e. positive slope.

Shown in Fig.3.15 are the event rates plotted as a function of the fill number for the blue (left) and yellow (right) polarimeters, respectively. The event rates show tendency to increase as the fill goes by. This trend is typically seen for the polarized proton beam operation at RHIC as a consequence of the continuous improvement of the accelerator performance throughout the RUN. Thus the increasing effective dead-layer thickness as a function of the rate as shown in Fig.3.14 can also be interpreted as a cumulated radiation damage effect.

A good way of decoupling the possible rate effect from the radiation damage one is to study a set of the beam profile scan data. Several dedicated polarization profile scan measurements were executed during Run05. Each measurement was performed to a few percent statistical precision in several steps transversely across the beam ${ }^{1}$. The event rate gets maximum when the target is positioned at the center of the beam, whereas the rate decreases

\footnotetext{
${ }^{1}$ More details about the profile measurements are discussed in Chapter 7
} 

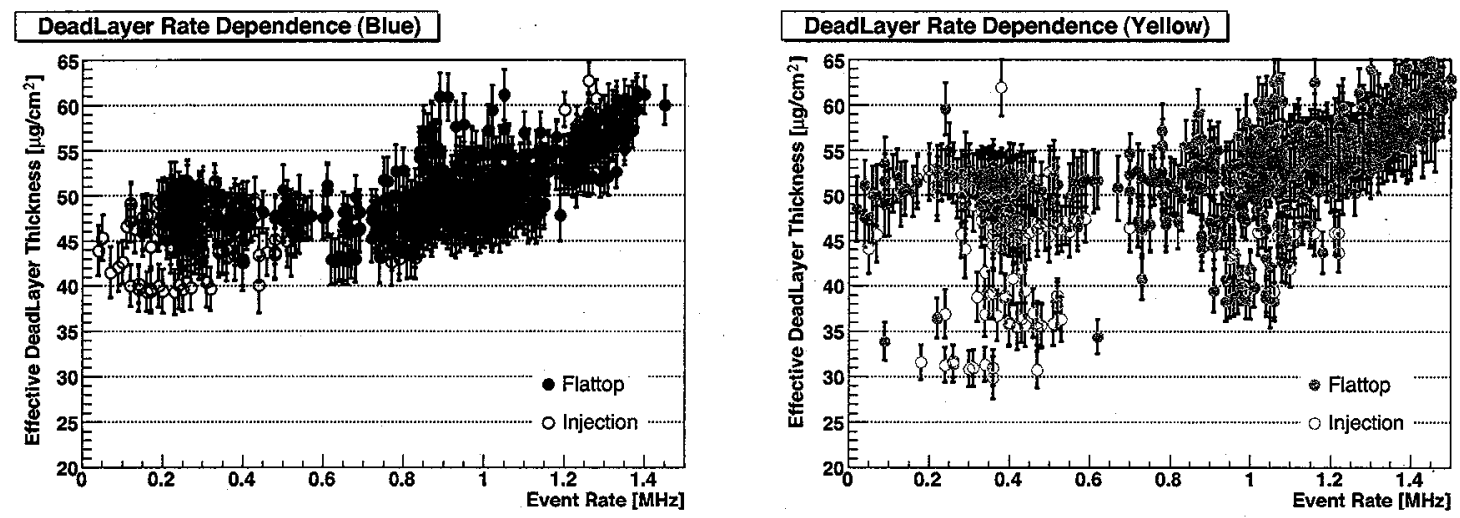

Figure 3.14: The effective dead-layer dependence on the event rate for blue (left) and yellow (right) polarimeters, respectively.
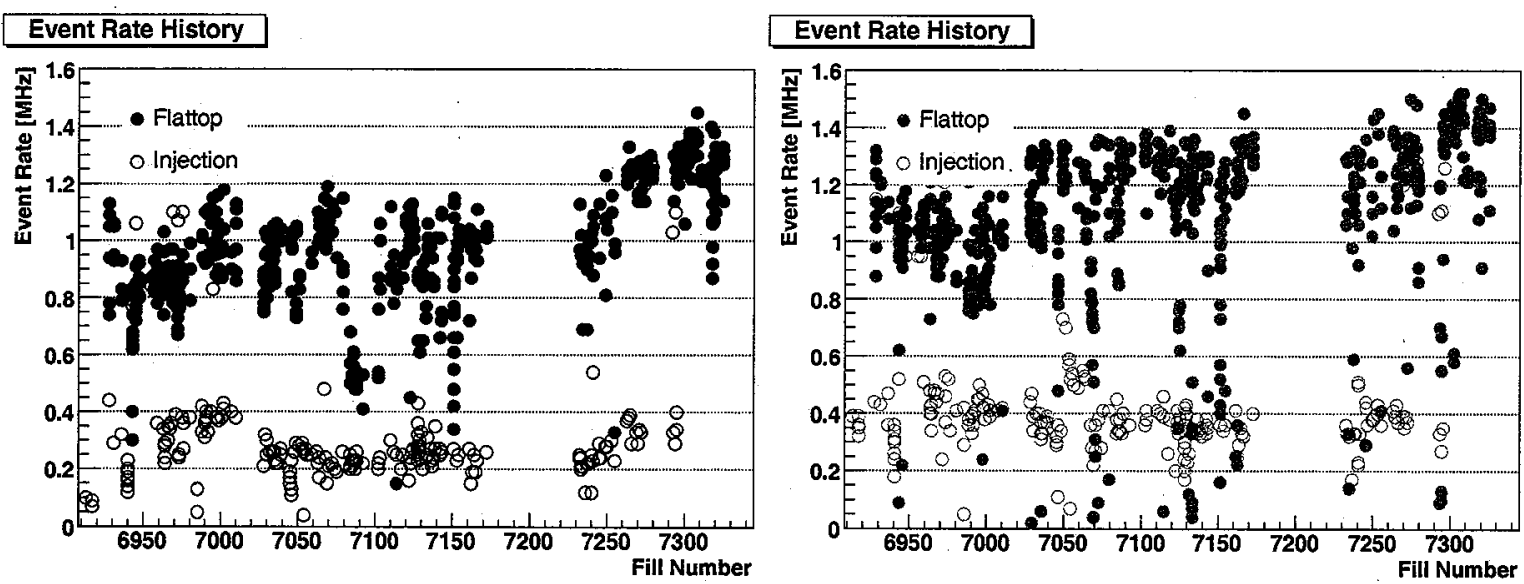

Figure 3.15: Event rates observed by blue (left) and yellow (right) polarimeters throughout Run05. The horizontal axis is the fill number. Solid and open circles represent measurements at the flat-top and injection energies. 
as the target is moved away from the beam center. An one set of profile measurement was executed one after another within the same fill. Within such a short period of time, it is safe to assume any change in the detector response should come from the rate, not from the the accumulated radiation damage. The accumulated radiation damage deteriorates the detector performance in much longer time scale. In addition, a set of profile measurements are carried out under the same beam intensity. Although the polarization measurements are destructive, but the beam loss in its intensity of each measurements are relatively minor. Thus any change in the effective dead-layer observed within a set of profile measurements can be interpreted as the effect of the average event rate.

Shown in the Fig.3.16 are the effective dead-layer thickness plotted as a function of event rate. The right panel shows the profile data taken during fill 7151 using the blue polarimeter, whereas left panel shows the data taken from 7133 and 7151 using the yellow polarimeter. All these profile scan measurements were done across the beam horizontally. Results of deadlayer fits for these measurements are summarized in Table. $3.1 \sim 3.3$. Regardless of the data sets for fill 7133 and 7151 measured using the yellow polarimeters overlap the range of the event rate from 0.15 to $1.1[\mathrm{MHz}]$, disagree by a few $\mu \mathrm{g} / \mathrm{cm}^{2}$. The cause of the discrepancy is not known, some difference in the experimental condition which is beyond our control besides the radiation damage effect. We assume the cumulating radiation damage effect only deteriorates the charge correction efficiency which affects on the effective dead-layer thickness towards only in increasing direction. Thus the cause of the discrepancy is unlikely originated from the radiation damage because the data in fill 7133 shows thicker $x_{\mathrm{dl}}$ than that of the later measurements in fill 7151. On the other hand, the behavior of the effective dead-layers seem quite similar regardless of data were taken in different fills, the blue and yellow polarimeters are completely independent detectors. All data sets show positive and shallow slopes below $1[\mathrm{MHz}]$ and start to get steeper beyond $1[\mathrm{MHz}]$.

Table 3.1: Run and polarization list of the profile scan executed in fill 7133 using the yellow polarimeter. The target position $x_{\text {tgt }}$ is given in the stepping motor pulse counts. 1 count $=$ $0.1 \mathrm{~mm}$. The effective dead-layer is given in the average of all active strips.

\begin{tabular}{cccccc}
\hline \hline RunID & $x_{\text {tgt }}[$ counts $]$ & $P[\%]$ & $\Delta P_{\text {sta }}[\%]$ & Rate $[\mathrm{MHz}]$ & $\overline{x_{\mathrm{dl}}} \pm \Delta x_{\mathrm{dl}}\left[\mu \mathrm{g} / \mathrm{cm}^{2}\right]$ \\
\hline 7133.106 & 1606 & -54.6 & 3.2 & 1.13 & $53.099 \pm 3.4 \times 10^{-2}$ \\
7133.107 & 1616 & -56.0 & 3.1 & 1.27 & $54.742 \pm 3.4 \times 10^{-2}$ \\
7133.108 & 1628 & -43.6 & 3.4 & 0.33 & $49.523 \pm 3.1 \times 10^{-2}$ \\
7133.110 & 1633 & -23.0 & 4.0 & 0.04 & $48.682 \pm 3.4 \times 10^{-2}$ \\
7133.112 & 1595 & -23.4 & 3.4 & 0.09 & $48.580 \pm 3.0 \times 10^{-2}$ \\
7133.113 & 1600 & -44.9 & 3.4 & 0.51 & $49.627 \pm 3.2 \times 10^{-2}$ \\
7133.114 & 1621 & -54.6 & 3.4 & 1.03 & $52.290 \pm 3.5 \times 10^{-2}$ \\
7133.115 & 1610 & -56.5 & 3.1 & 1.25 & $54.651 \pm 3.4 \times 10^{-2}$ \\
\hline \hline
\end{tabular}

A polynomial fit was applied on the data for fill 7133 taken using the yellow polarimeter as shown in the left panel of Fig.3.17. 
Table 3.2: Run and polarization list of the profile scan executed in fill 7151 using the blue polarimeter.

\begin{tabular}{cccccc}
\hline \hline RunID & $x_{\text {tgt }}[$ counts $]$ & $P[\%]$ & $\Delta P_{\text {sta }}[\%]$ & Rate $[\mathrm{MHz}]$ & $\overline{x_{\text {dl }}} \pm \Delta x_{\text {dl }}\left[\mu \mathrm{g} / \mathrm{cm}^{2}\right]$ \\
\hline 7151.007 & 1193 & -64.8 & 3.8 & 0.90 & $47.838 \pm 4.1 \times 10^{-2}$ \\
7151.008 & 1195 & -57.5 & 3.7 & 0.84 & $47.483 \pm 4.0 \times 10^{-2}$ \\
7151.009 & 1198 & -59.8 & 3.7 & 0.74 & $46.950 \pm 3.9 \times 10^{-2}$ \\
7151.010 & 1200 & -54.8 & 3.8 & 0.61 & $46.579 \pm 4.0 \times 10^{-2}$ \\
7151.011 & 1203 & -52.8 & 3.8 & 0.48 & $46.184 \pm 3.9 \times 10^{-2}$ \\
7151.012 & 1188 & -58.9 & 3.6 & 0.94 & $48.139 \pm 4.0 \times 10^{-2}$ \\
7151.013 & 1184 & -59.0 & 3.7 & 0.86 & $47.758 \pm 4.0 \times 10^{-2}$ \\
7151.014 & 1180 & -56.8 & 3.6 & 0.77 & $47.095 \pm 3.9 \times 10^{-2}$ \\
7151.015 & 1178 & -58.7 & 3.8 & 0.66 & $46.731 \pm 4.0 \times 10^{-2}$ \\
7151.016 & 1176 & -55.1 & 3.7 & 0.55 & $46.372 \pm 3.9 \times 10^{-2}$ \\
7151.017 & 1174 & -55.9 & 3.7 & 0.42 & $46.248 \pm 3.9 \times 10^{-2}$ \\
7151.018 & 1205 & -44.5 & 3.8 & 0.34 & $45.896 \pm 3.9 \times 10^{-2}$ \\
7151.019 & 1193 & -55.5 & 3.6 & 0.90 & $47.820 \pm 3.9 \times 10^{-2}$ \\
\hline \hline
\end{tabular}

$$
x_{\mathrm{dl}}=x_{0}+a \cdot r+b \cdot r^{2}
$$

where $r$ is the event rate, $x_{0}, a$ and $b$ are free parameters, where as $x_{0}$ is the effective dead-layer thickness at the event rate of zero, . The best fit parameters are presented in Table 3.4.

The data points of fill 7151 for both blue and yellow polarimeters are scaled by 7.5 and 3.5, respectively and superimposed on the fit as shown in the right panel of Fig.3.17. The scale factors were estimated by eye and the scaled data points are well fit on the curve. Thus the correlation function determined from one profile data sets seem compatible with other two data sets. The main purpose of this study is to evaluate the correlation function of the effective dead-layer which tells us the additional thickness with respect to the value at the event rate of zero.

So obtained rate correlation function was used to apply the rate dependent correction on the data points presented in the Fig.3.11. All data points were projected at the zero rate $(r=0)$ point.

Shown in the Fig. 3.18 are the results of the event rate correction using so obtained correlation function. All data points are projected to the event rate of zero. The discrepancy pointed out earlier between the data points taken at the injection and the store energies are now more or less vanished. The measurement at the injection energies were done only with the first 8 bunches, not full bunches thus event rates are typically lower than those of 
DeadLayers from Profile Runs (Blue)

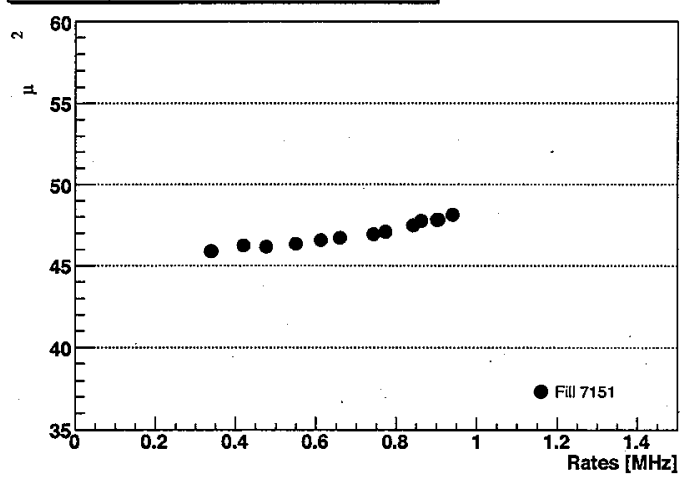

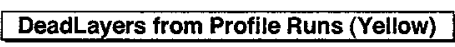

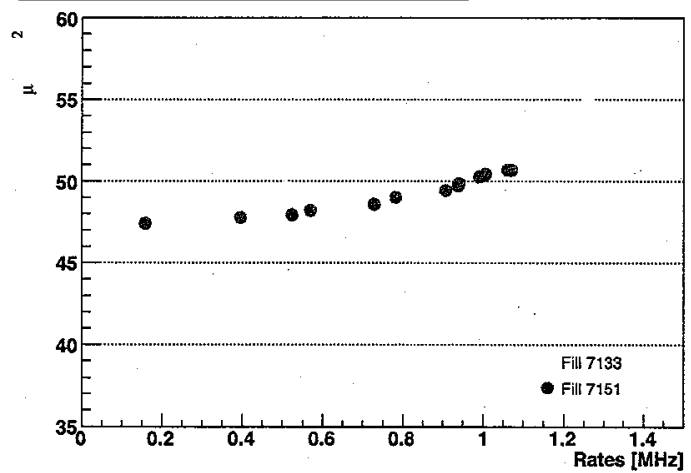

Figure 3.16: The event rate dependence of the effective dead-layer thickness. Data are from series of the polarization profile measurements executed during fill 7133 (left) using the blue and 7133 and 7151 using the yellow polarimeters.
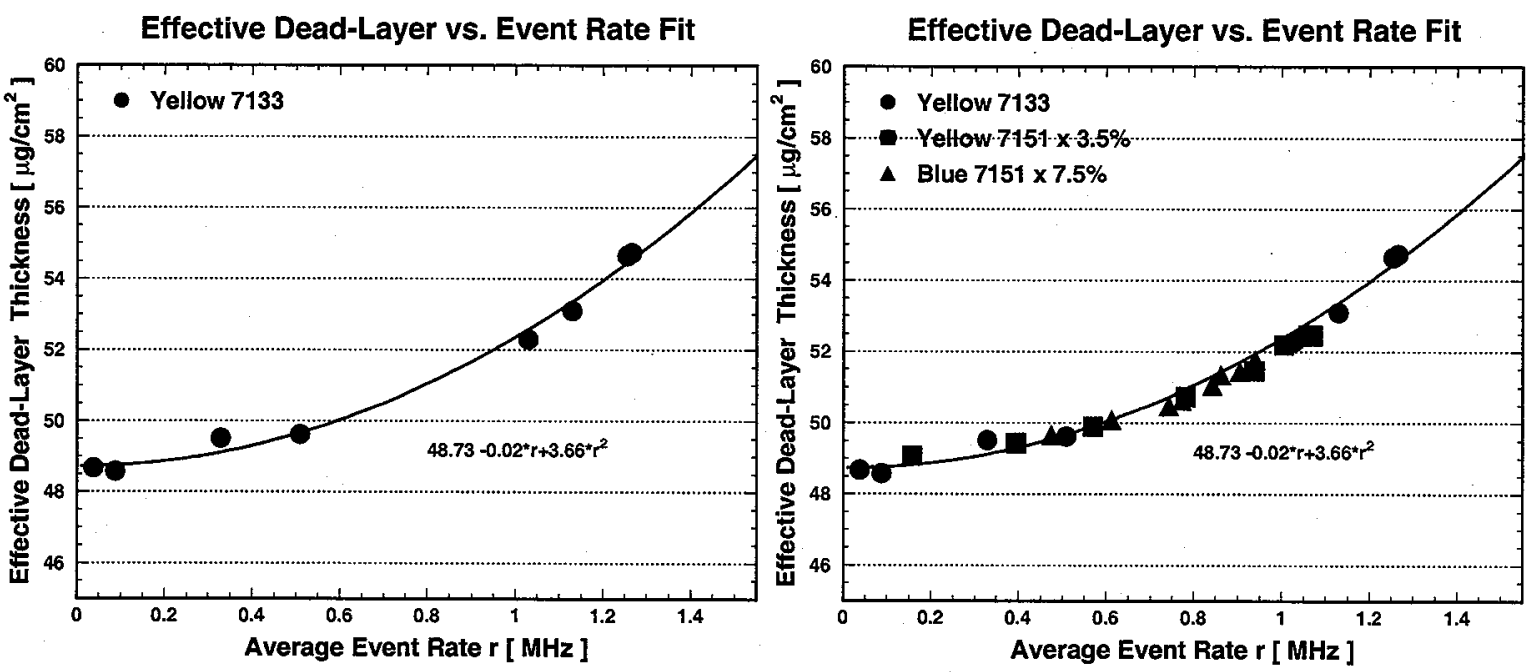

Figure 3.17: (left) Polynomial fit on the rate dependence of effective dead-layer observed during fill 7133 using yellow polarimeter. (right) Superimposed data points from fill 7151 on the data points of the left panel and the fitting curve. The absolute scale for the superimposed data points were adjusted by eye. 
Table 3.3: Run and polarization list of the profile scan executed in fill 7151 using the yellow polarimeter.

\begin{tabular}{cccccc}
\hline \hline RunID & $x_{\text {tgt }}[$ counts $]$ & $P[\%]$ & $\Delta P_{\text {sta }}[\%]$ & Rate $[\mathrm{MHz}]$ & $\overline{x_{\mathrm{dl}}} \pm \Delta x_{\mathrm{dl}}\left[\mu \mathrm{gg} / \mathrm{cm}^{2}\right]$ \\
\hline 7151.107 & 1611 & -25.1 & 3.6 & 0.94 & $49.706 \pm 2.9 \times 10^{-2}$ \\
7151.108 & 1614 & -29.1 & 3.7 & 0.78 & $49.003 \pm 2.9 \times 10^{-2}$ \\
7151.109 & 1616 & -16.5 & 3.7 & 0.57 & $48.195 \pm 2.8 \times 10^{-2}$ \\
7151.110 & 1620 & -10.3 & 3.7 & 0.40 & $47.750 \pm 2.8 \times 10^{-2}$ \\
7151.111 & 1623 & -4.2 & 3.8 & 0.16 & $47.406 \pm 2.7 \times 10^{-2}$ \\
7151.112 & 1609 & -38.4 & 3.5 & 1.00 & $50.406 \pm 2.9 \times 10^{-2}$ \\
7151.113 & 1606 & -40.4 & 3.6 & 1.06 & $50.682 \pm 3.1 \times 10^{-2}$ \\
7151.114 & 1603 & -33.6 & 3.6 & 1.07 & $50.666 \pm 3.0 \times 10^{-2}$ \\
7151.115 & 1600 & -33.9 & 3.5 & 0.99 & $50.279 \pm 2.9 \times 10^{-2}$ \\
7151.116 & 1596 & -28.6 & 3.6 & 0.91 & $49.421 \pm 2.9 \times 10^{-2}$ \\
7151.117 & 1593 & -26.8 & 3.7 & 0.73 & $48.565 \pm 2.8 \times 10^{-2}$ \\
7151.118 & 1590 & -17.7 & 3.7 & 0.52 & $47.932 \pm 2.8 \times 10^{-2}$ \\
7151.119 & 1610 & -25.3 & 3.6 & 0.94 & $49.866 \pm 2.9 \times 10^{-2}$ \\
\hline \hline
\end{tabular}

Table 3.4: The best fit parameters of the polynomial fit on the event rate dependent effective dead-layer data.

\begin{tabular}{cc}
\hline \hline$x_{0}$ & $48.73 \pm 0.25 \times 10^{-1}$ \\
$\mathrm{a}$ & $-0.02 \pm 0.10$ \\
$\mathrm{~b}$ & $3.66 \pm 0.75 \times 10^{-1}$ \\
\hline \hline
\end{tabular}

measurements at the store energy. Thus the correction brought down the data points at the store energy more and the injection energy less.

\subsubsection{6 and 112 Bunch Modes Effect}

The beam bunches can be filled up to 112 bunches at the maximum in the RHIC beam operation out of 120 total bunch spacings. Each beam bunches were separated by 102 ns. When the beam was operated below 56 bunches ( 56 bunches mode), the beam was filled every other bunches on purpose. In experimental point of view, it remains less uncertainties to identify the bunches for events should belong if every filled bunches are followed by empty ones. Not only the experiments, but also the RHIC polarimeters take the advantage of this. The timing gate window was kept wider, i.e. $160 \sim 180$ ns for 56 bunches operation, whereas about 60 ns for more than 56 bunches (112 bunch mode) as shown in Fig.??. Clearly the energy range to be accumulated for 56 bunches mode was wide, however the energy range 

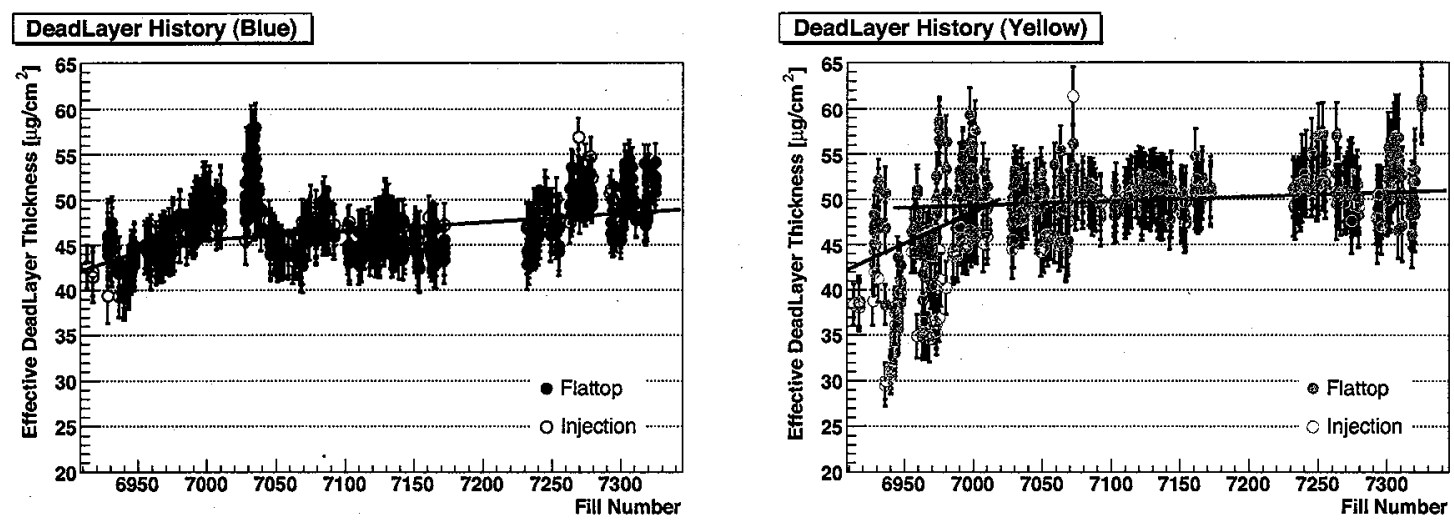

Figure 3.18: The effective dead-layer thickness history as Fig.3.11, but the data points are corrected for the average event rates.

to be analyzed for the polarization analysis was covered by both modes. This energy range was fixed and not altered between the data taken with 56 bunches mode or others.

56 bunch mode

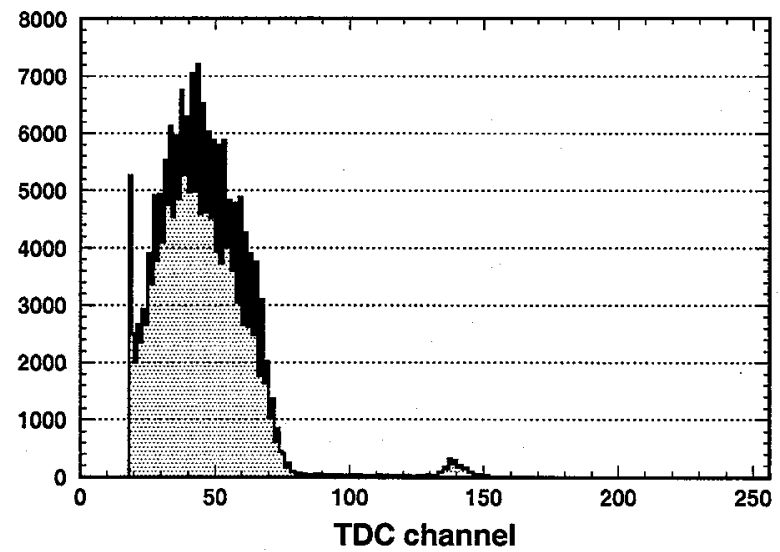

112 bunch mode

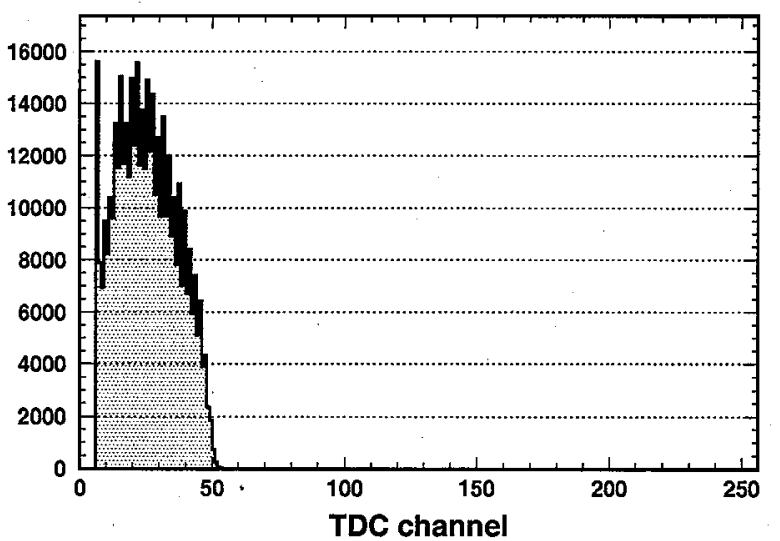

Figure 3.19: Typical raw TDC spectra for 56 bunch (left) and 112 bunch (right) modes. 1 TDC count $=1.1845 \mathrm{~ns}$.

Run05 was the first beam operation with more than 56 bunches and the first attempt of the 112 bunches mode was occurred fill number 7021 as shown in Fig. 3.20 and Fig. 3.21. This is about the time the slope, i.e. the increasing effective dead-layer thickness trend as pointed out in the beginning of this section, changed to shallower increase. Although determination (or prediction) of the slope change timing is not too sharp from the empirical fits in Fig.3.11, the timing seem to match with the mode change to the 112 bunches mode whether it is an accidental or not. However, it is non-trivial to claim the change in the timing gate to be the cause of slope change in the effective dead-layer thickness, because the energy range of 
the data fed into the dead-layer fit are not altered between two different operation modes. In other word, any changes between these two different modes were occurred out side of the energy range quoted for the effective dead-layer and polarization analysis. Despite of many sorts of correlation study performed in offline, we could not find any evidences of the slope change in the effective dead-layer history caused by the altering operation mode.
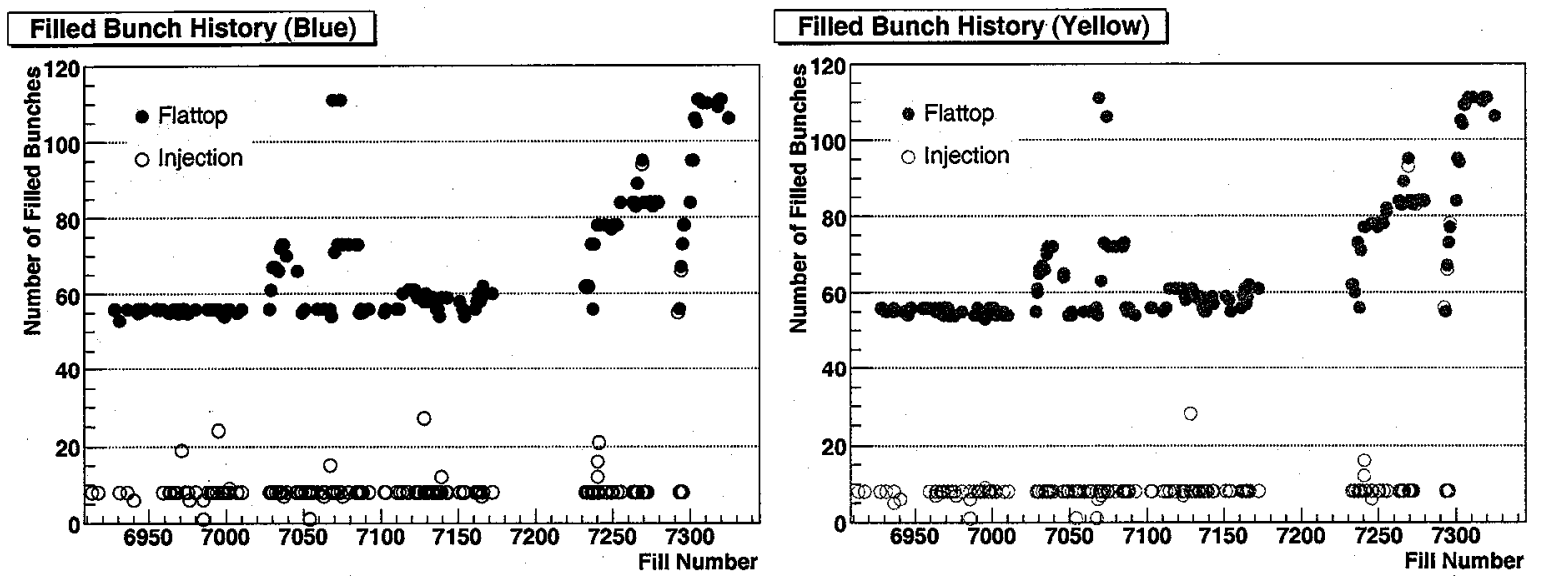

Figure 3.20: Number of filled bunches plotted as a function of the fill number for blue (left) and yellow (right) beams. Solid and open circles represent data points at the flat-top and injection energies, respectively.

\subsubsection{Drifting $t_{0}$}

Shown in the Fig.3.22 are the $\overline{t_{0}}\left(t_{0}\right.$ averaged over all active strips) observed in the blue (right) and yellow (left) polarimeters. Data are plotted for not only physics stores, but also non-physics stores. Horizontal axis is the fill number. There are absolute timing difference between 56 bunches and 112 bunches modes and it is adjusted by shifting data points of 112 bunches mode by $+14 \mathrm{~ns}$ for blue and $+18 \mathrm{~ns}$ for yellow polarimeter measurements. Shallow, but very much linear drifts are seen in both blue and yellow polarimeters. The overall drift is about 5 ns over the course of 3 monthes of the Run05 running period.

The solid squares plotted in Fig.3.22 are the peak intensity timing of the bunch observed by the wall current monitor[16]. The timing is estimated by eye from the longitudinal profile distributions combined for all bunches as shown in the right panel of Fig. 3.23. As can be seen from the Fig. 3.22, the timing drift was not observed in the wall current monitor. Thus we didn't find such the slow but constant timing drift in other device/monitors than the proton-Carbon polarimeters.

A possible candidate to be the timing drift can be a temperature effect of a beam clock cable. The temperature dependence of this cable is $10 \mathrm{ps} / \mathrm{km} /{ }^{\circ} \mathrm{C}$. Although the precise distance of this cable length is unmeasured, nevertheless the rough estimate of $4 \mathrm{~km}$ is still 

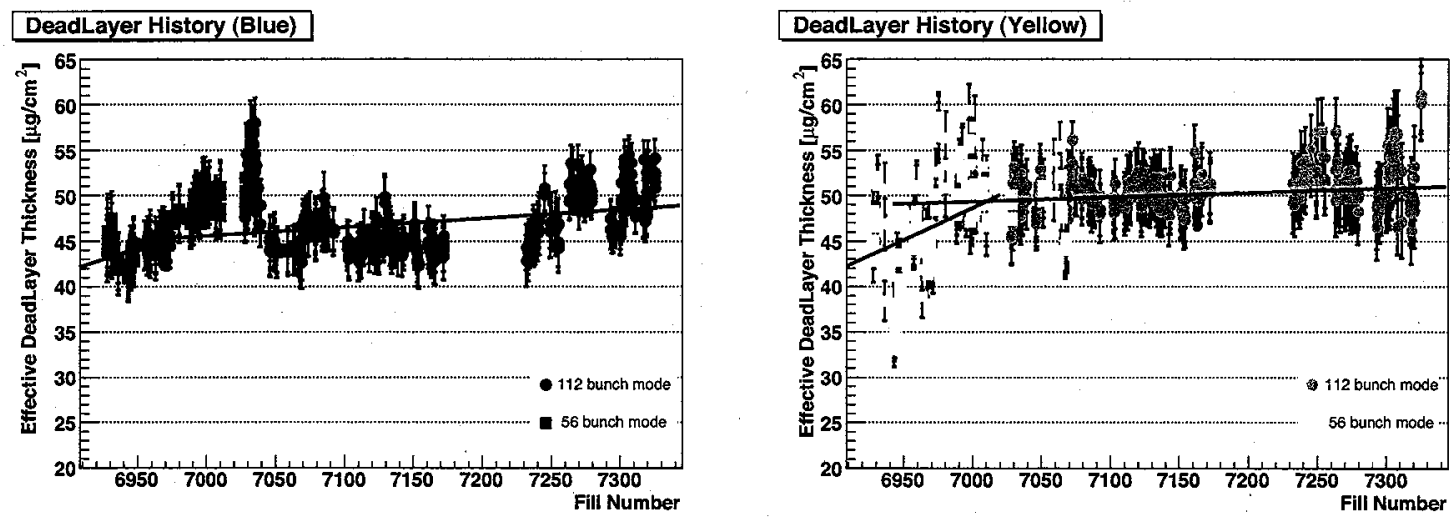

Figure 3.21: The (rate corrected) effective dead-layer histories for blue (left) and yellow (right) polarimeters distinguished data points by the measurement performed in the 56 (solid square) and 112 (solid circle) bunch modes, respectively.
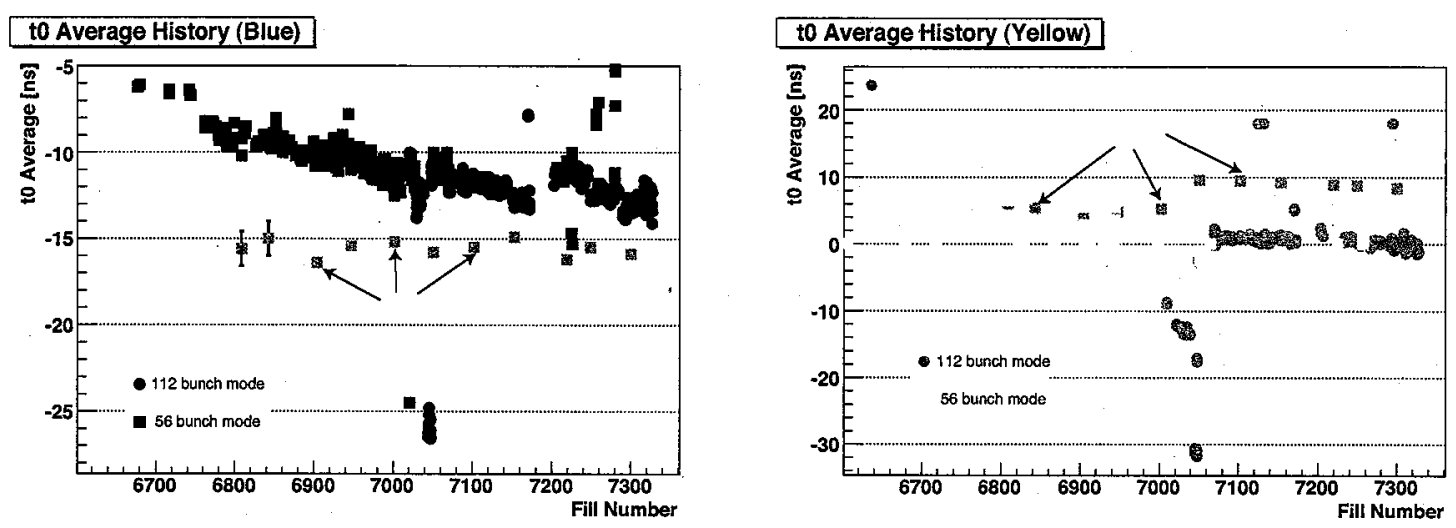

Figure 3.22: $\overline{t_{0}}$ plotted as a function of fill number for blue (right) and yellow (left) polarimeters, respectively. The absolute timing difference between 56 bunches and 112 bunches modes were adjusted by shifting data points of 112 bunches mode by $+14 \mathrm{~ns}$ for blue and $+18 \mathrm{~ns}$ for yellow polarimeter measurements. Solid square represent the timing of the wall current monitor[16]. Data points fill number between 7203 and 7226 in the left panel are from the operation with the beam energy of $205 \mathrm{GeV}$. The polarimeter was under frequent tune during this period. 

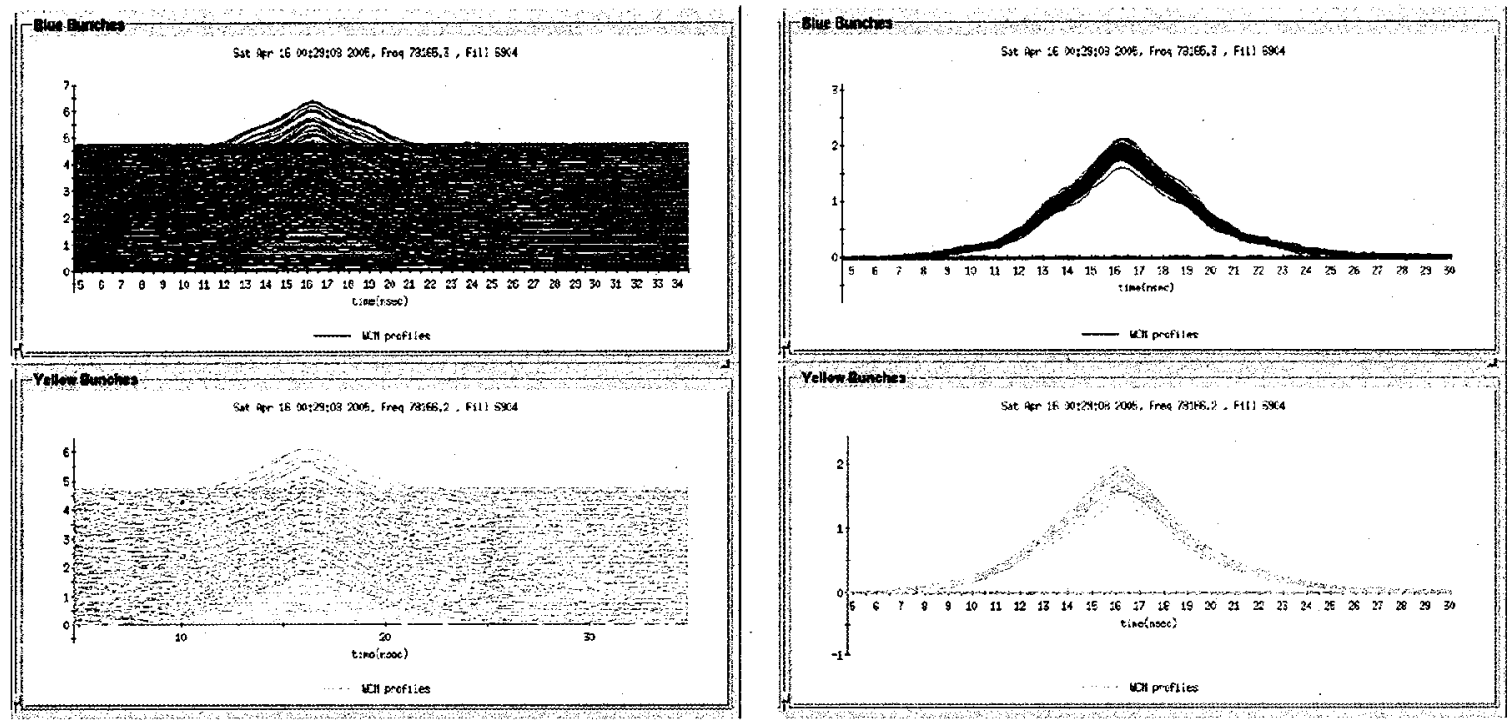

Figure 3.23: The longitudinal beam profile measured by the wall current monitor during fill 6904 for blue (top) and yellow (bottom) beams. Each curve corresponds to each bunch. The left panel is the projection of right panel onto a $\mathrm{x}-\mathrm{y}$ plane. The horizontal and vertical axes represent the intensity and time.

factor of 5 short to explain $5 \mathrm{~ns}$ drift out of $20^{\circ} \mathrm{C}$ change in the temperature. From the fact, it is unlikely the $5 \mathrm{~ns}$ cannot be caused, at least solely, by the temperature effect.

Another possibility is the radiation damage of the beam clock cable. This possibility was not investigated in Run05 analysis.

In theory, two free parameters $x_{\mathrm{dl}}$ and $t_{0}$ in the dead-layer fitting are completely independent parameters. However, one may concern that the tendency of increasing thickness in $x_{\mathrm{dl}}$ and $t_{0}$ drift have a correlation. Such a correlation can occur under the circumstance that the applying $d E / d x$ model is irrelevant, for instance.

Thus $\overline{t_{0}}$ problem remains unsolved.

\subsection{Systematic Uncertainties}

Regardless of many efforts made to understand the behavior of the effective dead-layer, not all phenomena have been explained. Unexplained behaviors are assigned systematic errors in the present subsection.

As guided by the solid line in Fig.3.24 the effective thickness of the dead-layer is still growing as measurements go by even after the event rate corrections were made. Now the guiding line is applied to all fill range, since we couldn't find out any reason that the slope could be changed at the transition between 56 and 112 bunches mode. As illustrated in Fig. 3.25, this growth of inefficiency can be interpreted as the deteriorated charge correction 
efficiency as a consequence of cumulated radiation damage in the surface region. Besides the precision limit of the fitting, the cause of varying dead-layer thickness is not known at this time. Also possible sources are change in the electronics noise or a baseline shift, which are not accounted in the present model. These fluctuations are thus accounted as uncertainty for the individual measurement.
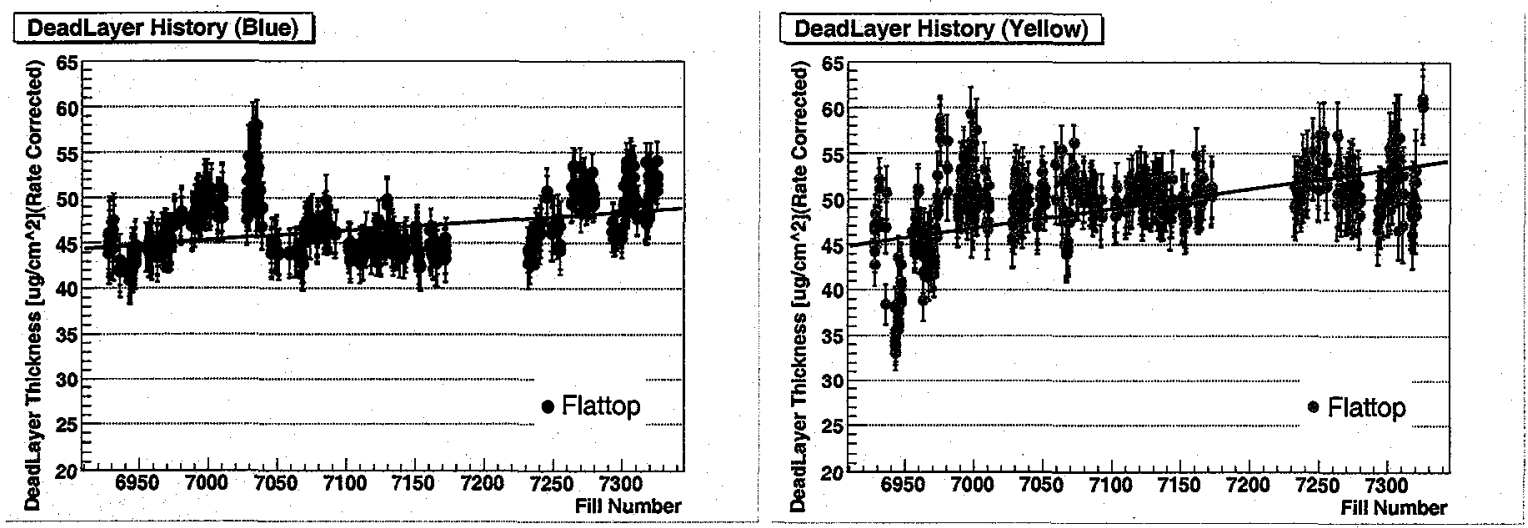

Figure 3.24: Same plots with Fig. 3.18, but a single linear fit was applied to all range of data.

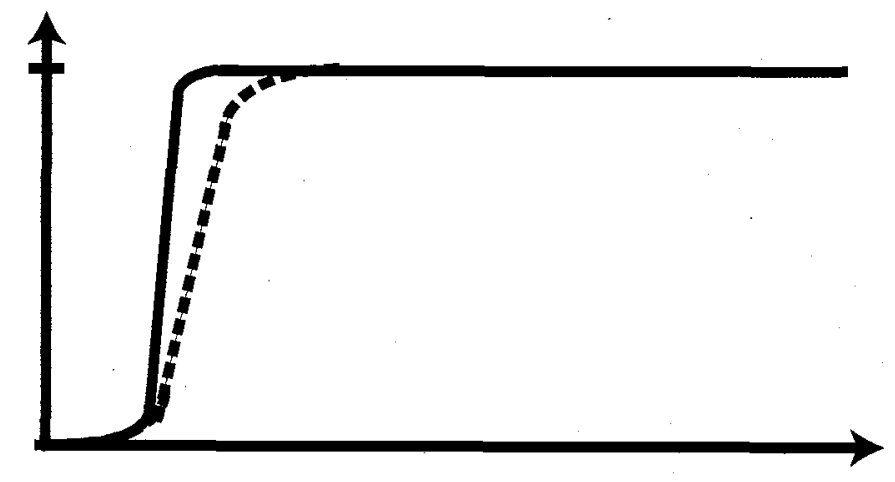

Figure 3.25: Charge collection efficiency as a function of the depth of the silicon detector. The dashed curve images the deteriorated charge correction efficiency due to the radiation damage compared to the one before the damage (solid curve).

The fluctuation in Fig. 3.24 can be quantified as $\sigma$ width of the distribution modeled by the Gaussian shape. Assuming the shallow growth of the effective dead-layer as a function of time is really caused by the radiation damage, the data were projected on the perpendicular axis to the slopes. The projected distributions are shown in Fig. 3.26 as a consequence of 
the radiation damage correction. These are all correction we can apply as known sources of the effective dead-layer behavior. Taking $1 \sigma$ of the distributions in Fig. 3.26, $\Delta x_{\mathrm{dl}}=2.6$ and $2.9 \mu \mathrm{g} / \mathrm{cm}^{2}$ were estimated for the blue and the yellow polarimeters, respectively.
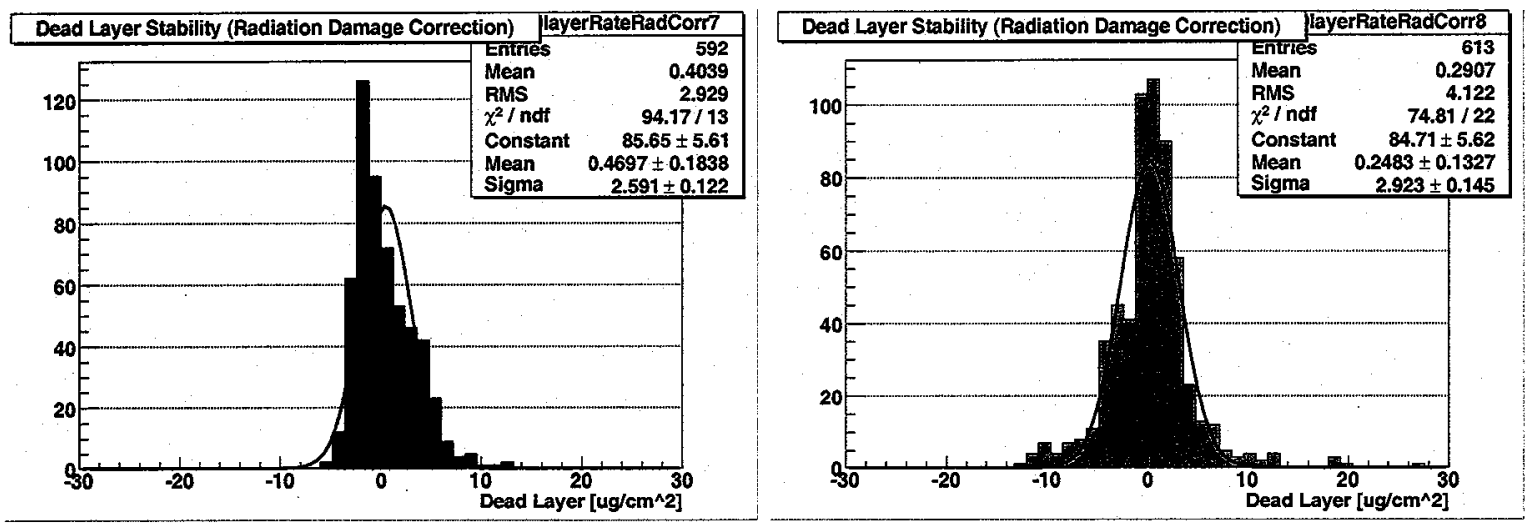

Figure 3.26: The effective dead-layer distributions for blue (left) and yellow (right) polarimeter measurements after the radiation damage correction. Data sets are same as presented in Fig. ??.

The impact of the uncertainties in the effective dead-layer $\Delta x_{\mathrm{dl}}$ on the polarization was studied by calculating the polarizations with on purposely scaled effective dead-layers. As shown in Fig. 3.27, the polarizations were calculated with the additional/subtractive effective dead-layer thickness of $\pm 30 \mu \mathrm{g} / \mathrm{cm}^{2}$ with respect to the nominal values. The calculations were made every 5 to $10 \mu \mathrm{g} / \mathrm{cm}^{2}$ steps for three arbitrarily selected runs 7295.005 (left), 7279.106 (middle), and 6943.105 (right), respectively. As can be seen, the resulting polarizations show reasonably linear responses against the effective dead-layer thickness within the range of $\pm 20 \mu \mathrm{g} / \mathrm{cm}^{2}$, and they are consistent within sampled runs, i.e. $0.27,0.28$, and $0.23 \% /\left[\mu \mathrm{g} / \mathrm{cm}^{2}\right]$, respectively. Pessimistically taking the worse case, i.e. $0.28 \% /\left[\mu \mathrm{g} / \mathrm{cm}^{2}\right]$ for the yellow polarimeter measurements, the uncertainty of the effective dead-layer of $\Delta x_{\mathrm{dl}}=$ 2.6 (blue) and 2.9 (yellow) $\mu \mathrm{g} / \mathrm{cm}^{2}$ polarimeter measurements are translated to the uncertainty in the polarizations $\Delta P^{\mathrm{dl}}$ of 0.7 (blue) and $0.8 \%$ (yellow) in the absolute scale, 1.5 and $1.7 \%$ in the relative scale $\left(\Delta P^{\mathrm{dl}} / P\right)$.

Note an additional/subtractive effective dead-layer will destroy the kinematic relations between the kinetic energy and the time-of-flight to reconstruct the carbon mass at the right location. In order to apply the relevant event selection to throughout the dependence study data, the timing offset was tuned by corresponding amount for the carbon mass peak to be reconstructed at the right location. 

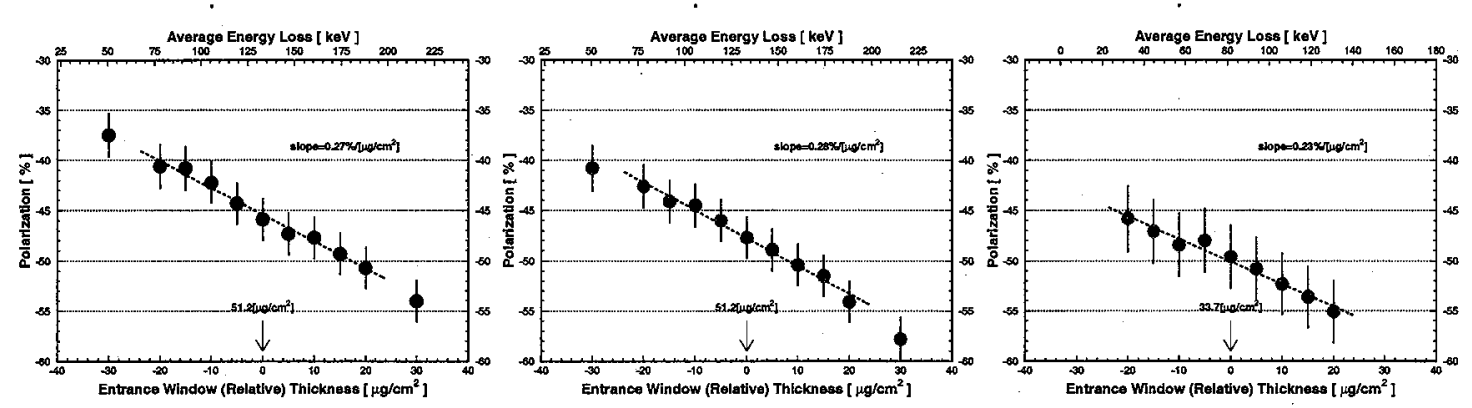

Figure 3.27: The polarization dependence on the effective dead-layer thickness for the runs 7279.005 (left), 7279.106 (middle), and 6943.105 (right), respectively. 


\section{Chapter 4}

\section{CONFIGURATION FILE AND EVENT RECONSTRUCTION}

\subsection{Configuration File}

Thus the dead-layer fit was applied for all strips of all measurements and resulting best fit parameters were summarized and kept as a configuration file. This is fully automated procedure. The next step is to reconstruct the kinematics based on these configuration database. In principle, it is desirable to use the configuration file which was produced based on the run whose kinematics to be reconstructed. However this simple principle didn't applied for the Run05 analysis because of the failure of dead-layer fit to some fraction of data mostly caused by the higher background conditions or the electronics failures. Loading such a configuration file contains inadequate fitting results will leads to wrong polarization results. Here a flexible logic was introduced in order to avoid the polarization results to be driven by the bad dead-layer fit.

Given the fact we set our goal to the precision of the effective dead-layer to be $5 \mu \mathrm{g} / \mathrm{cm}^{2}$, applying configuration file determined from other run would not introduce additional errors if they are consistent to each other within the $3 \mu \mathrm{g} / \mathrm{cm}^{2}$. To be more concrete, the detector averaged effective dead-layer thicknesses are compared for all 6 detectors to determine if two configuration files are consistent or not. This way, the strips whose dead-layer fit was failed can still be reconstructed kinematics in a reasonable manner by employing the configuration file which is evaluated previously, perhaps before the electronics failure, for example. Once there is a reliable configuration file, apply it to consecutive runs as long as the configurations are consistent within $3 \mu \mathrm{g} / \mathrm{cm}^{2}$. This procedure is certainly an advantage not to loose the precision for low statistics runs, whose precision of dead-layer fit directly limited by the statistical abundance. Applying the dead-layer configuration file which is determined from high statistics data seems reasonable under the assumption the dead-layer wouldn't jump too drastically in a short period of time. Fig. 4.1 show how frequently the configuration files were updated throughout the analysis. Superposed by the solid triangle data points are the data which employ the configuration file made by own data. Data points which are not 
superposed employ the configuration file made by previous runs.
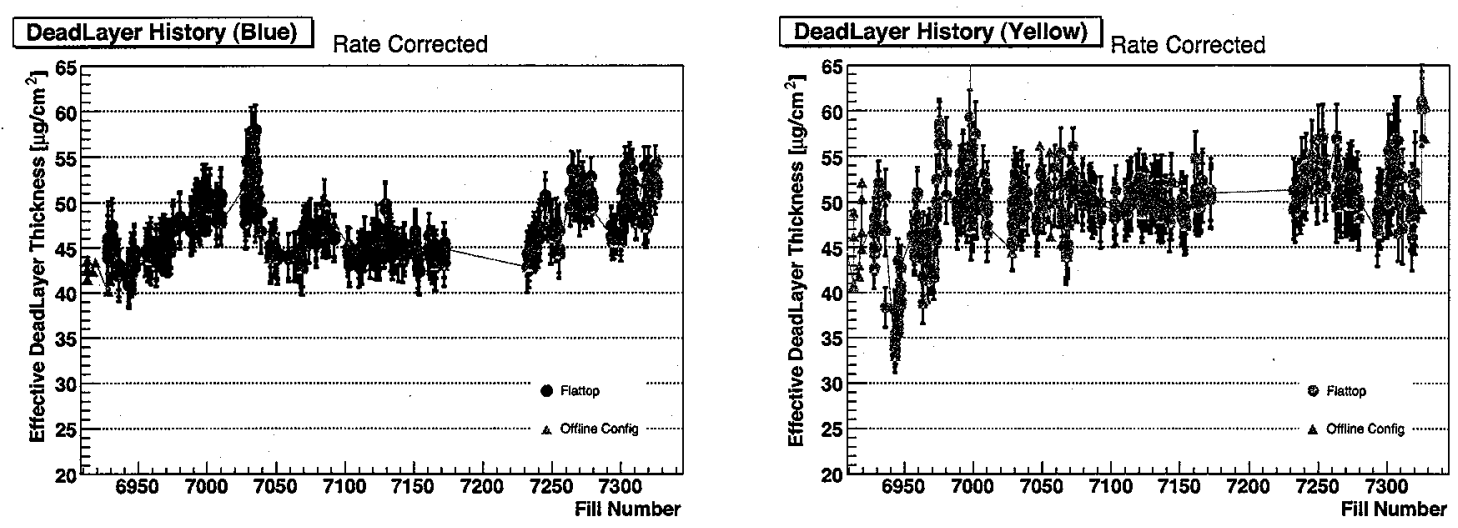

Figure 4.1: The effective deal-layer history plots for the blue (left) and yellow polarimeters as same as Fig. 3.24, but superposed by the solid triangles which represent the configuration file update.

The consistency in $t_{0}$ is not checked in above process. $t_{0}$ is adjusted after configuration file to be used is selected. At the really beginning of the kinematics reconstruction program, events are sparcified by factor of 100 (just to speed up, sacrificing the statistical precision) and re-calculate the $t_{0}$ to locate the invariant mass peak to be at the carbon mass based on the current effective dead-layer thicknesses. This is called "feedback mode" in the program.

\subsection{Event Selection}

\subsubsection{Energy Range}

As it is described in the introduction, extending the energy range lower region is certainly an advantage in terms of 1 ) the larger magnitude of $A_{\mathrm{N}}$ in lower $-t$ and 2) larger cross section (more statistics). However, the practical range is limited by the tolerable size of the uncertainty to reconstruct the energy in the low energy region.

Shown in the Fig. 4.2 is the residual of the typical best effective dead-layer fits and the data for given strips (data - fit). The data are the peak positions estimated by the Gaussian fit on each sliced energy bins within (10 keV/bin, 50 bins) the energy range $400 \leq E \leq 900$ $\mathrm{keV}$ ) of the energy vs. ToF correlation plot. As it is briefly discussed in the Section 3.3.4, the current $d E / d x$ model fits very well in the region $E \geq 500 \mathrm{keV}$ (residual $\sim 0$ ), while it tends to undershoot the data $E<500 \mathrm{keV}$ (residual $<0$ ). Typically the residual gets about $-0.5 \mathrm{~ns}$ at $E=400 \mathrm{keV}$ and rare, but worst case, -1 ns at $E=900 \mathrm{keV}$. Such a systematic tendency suggests an inconsistency between current $d E / d x$ model with data. Thus we set the lower energy limit to be $400 \mathrm{keV}$ whose corresponding error is studied below. 

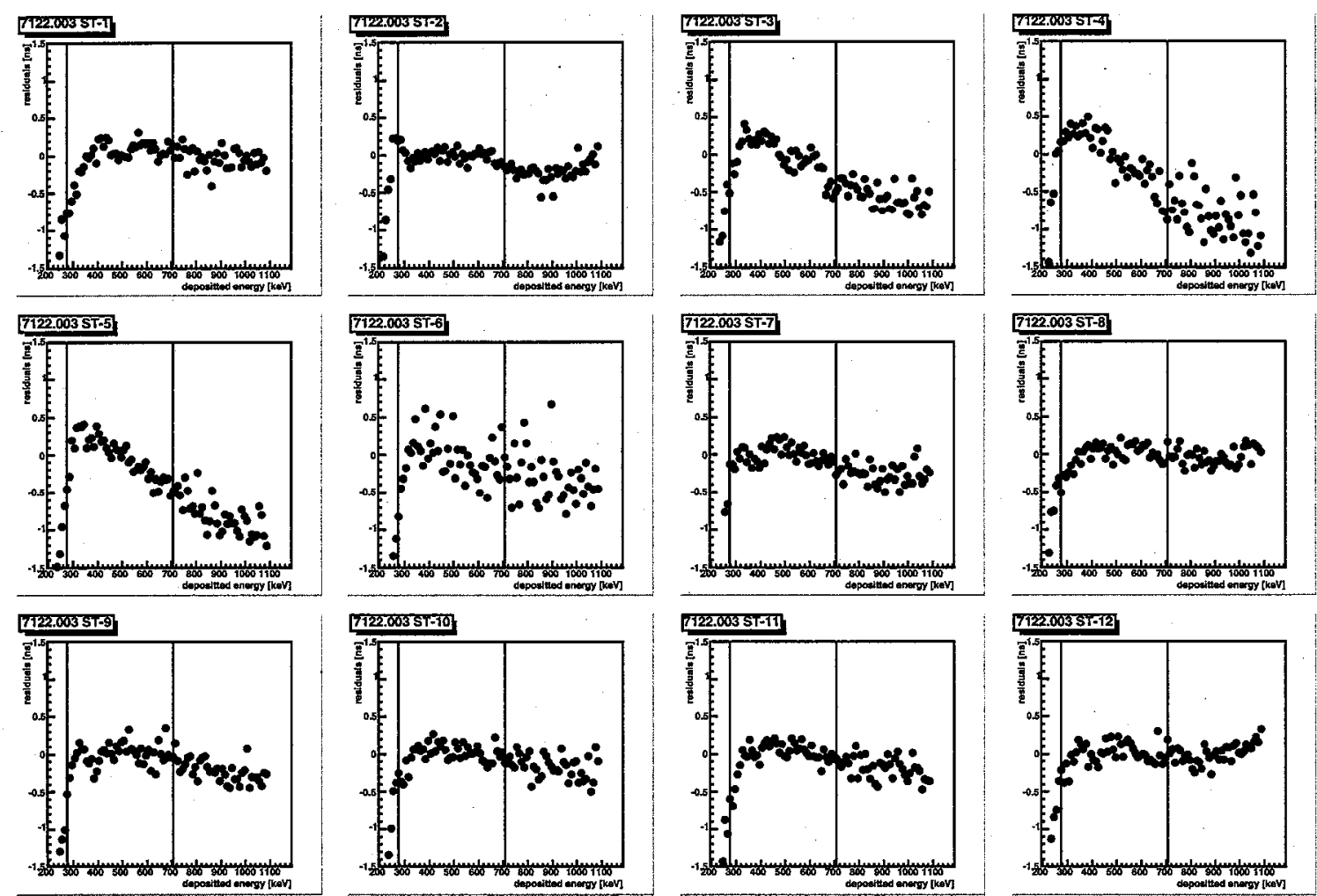

Figure 4.2: Residual plots for several strips. The vertical and horizontal axes represent residual $t$ ns and the measured energy $E_{\text {meas. }}$ in $\mathrm{keV}$. The vertical lines show corresponding kinetic energy of $E=400$ and $900 \mathrm{keV}$. 
The error of $\pm 1 \mathrm{~ns}$ in $t_{0}$ determination can be calculated by taking the derivative of kinetic energy formula by $t$ :

$$
\begin{aligned}
E & =\frac{1}{2} M \frac{L^{2}}{t_{\mathrm{tof}}^{2} c^{2}} \\
\frac{\partial E}{\partial t_{\mathrm{tof}}} & =-M \frac{L^{2}}{t_{\mathrm{tof}}^{3} c^{2}}=-\frac{2 \sqrt{2} c}{L \sqrt{M}} E \sqrt{E}
\end{aligned}
$$

Shown in Fig. 4.3 is the $\partial E / \partial t_{\text {tof }}$ in the unit of $\mathrm{keV} / \mathrm{ns}$ plotted as a function of $E$. As can be seen, the effects are about $11 \mathrm{keV}$ at $E=400 \mathrm{keV}$ and $37 \mathrm{keV}$ at $E=900 \mathrm{keV}$ (approximately corresponding effective dead-layer is $4 \mu \mathrm{g} / \mathrm{cm}^{2}$ ). Thus overall uncertainty due to the precision of $t_{0}$ determination by \pm results in the energy determination of $3 \sim 4$ $\%$ within the energy range $400 \leq E \leq 900 \mathrm{keV}$.

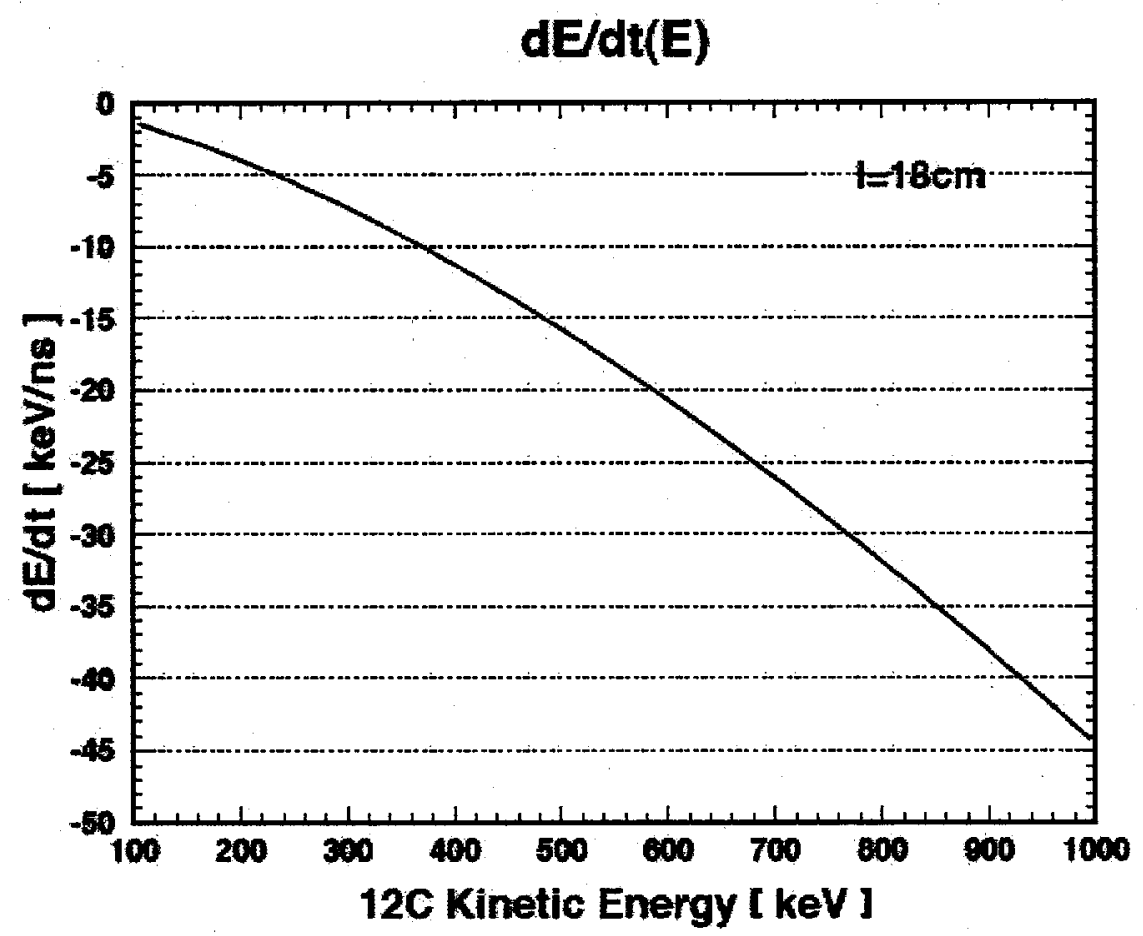

Figure 4.3: $\partial E / \partial t_{\text {tof }}$ in the unit of $\mathrm{keV} / \mathrm{ns}$ plotted as a function of $E$.

\subsubsection{Invariant Mass Cut}

Shown in the Fig. 4.4 is the typical time-of-flight and the kinetic energy plot reconstructed using the best fit parameters of the dead-layer fit. Dotted and solid curves represent the 
$2 \sigma$ and $3 \sigma$ cuts of the invariant mass as shown in Fig.4.5. The small peak seen below 4 $\mathrm{GeV}$ consisted of $\alpha$ backgrounds whose peak does not necessarily appear at the right $\alpha$ mass because the energy loss in the effective dead-layer was calculated assuming the carbon ion mass. A contamination of the $\alpha$ background underneath the carbon invariant mass peak is typically less than $1 \%$ within $3 \sigma$ from the nominal carbon mass position. Within $3 \sigma$ cuts, the number of the elastic carbons are observed about $200 \sim 300$ thousand events. About $50 \%$ of accumulated events from raw data were dropped after the energy and the $3 \sigma$ cuts were applied.

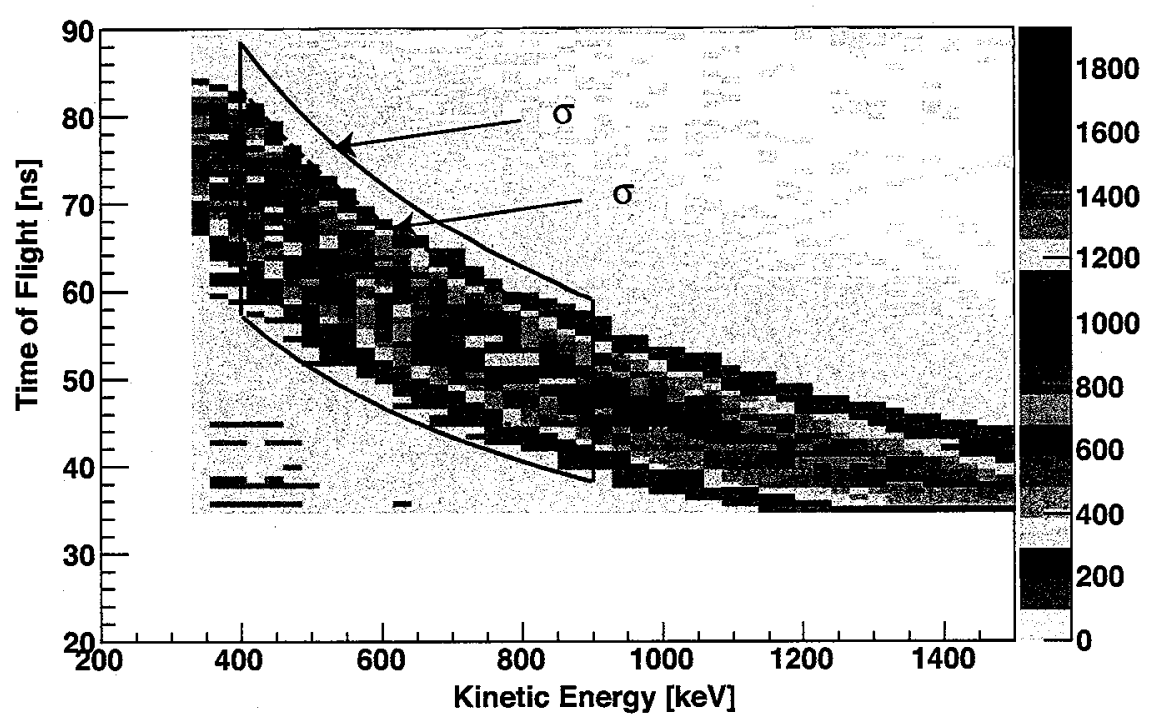

Figure 4.4: Time of flight and reconstructed kinetic energy correlation plot after the energy correction. Dotted and solid curves show 2 and $3 \sigma$ from carbon mass in the invariant mass distribution.

The effect of the backgrounds contamination underneath the carbon invariant mass was studied by comparing the polarizations between $2 \sigma$ and $3 \sigma$ invariant mass cuts. Shown in Fig. 4.6 are ratio of the polarizations calculated by $2 \sigma$ and $3 \sigma$ invariant mass cuts for blue (left) and yellow (right) polarimeters. As can be seen in the figure, the polarizations derived by the $2 \sigma$ cut are about $1 \%$, higher than the $3 \sigma$ cut. This result suggests the more background contamination in $3 \sigma$ cut case drives the lower polarization.

The exterpolation of the polarization at the $0 \sigma$ cut is necessary in order to evaluate the polarization under the circumstances of zero background contamination. However, this is not necessary in our case because this effect is absorbed into the "effective" analyzing power of proton-Carbon as a consequence of the normalization against the H-Jet average polarization. This is valid as long as we keep the $\sigma$ cut of the invariant mass consistent with data goes to H-Jet normalization and polarizations provided to experiments. In other word, the analyzing power determined by the normalization is "optimized" to make the average polarization measured by the proton-Carbon polarimeter to be consistent with the 


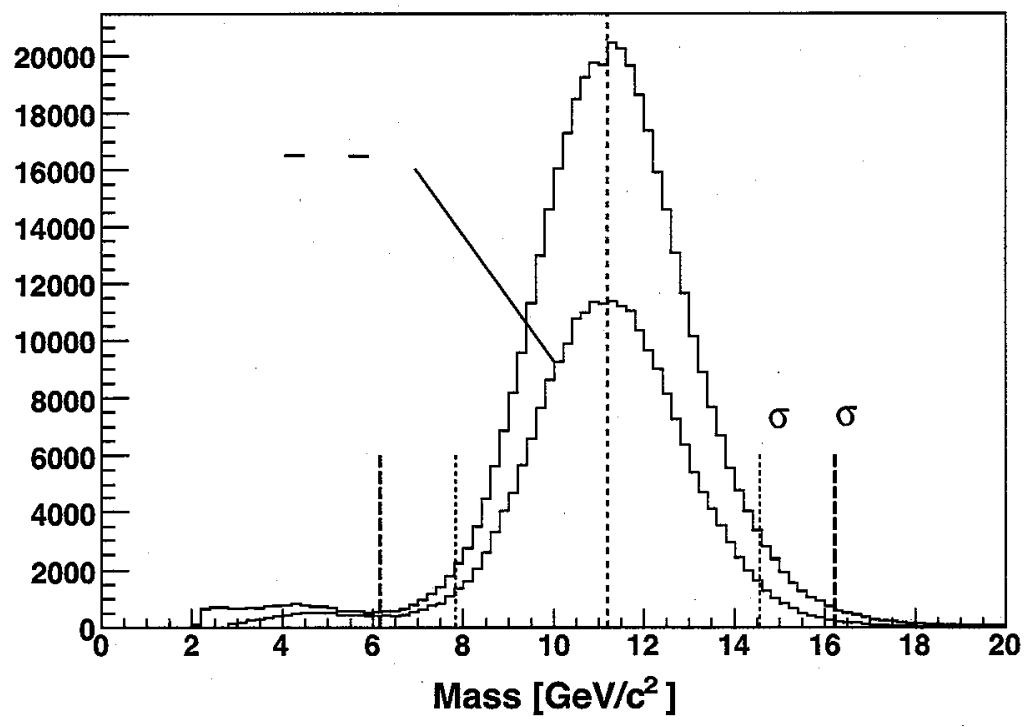

Figure 4.5: A typical invariant mass distribution. The red histogram shows the invariant mass for the events $400 \leq E \leq 900 \mathrm{keV}$. Dotted and dashed lines represent 2 and $3 \sigma$ from carbon mass, respectively.
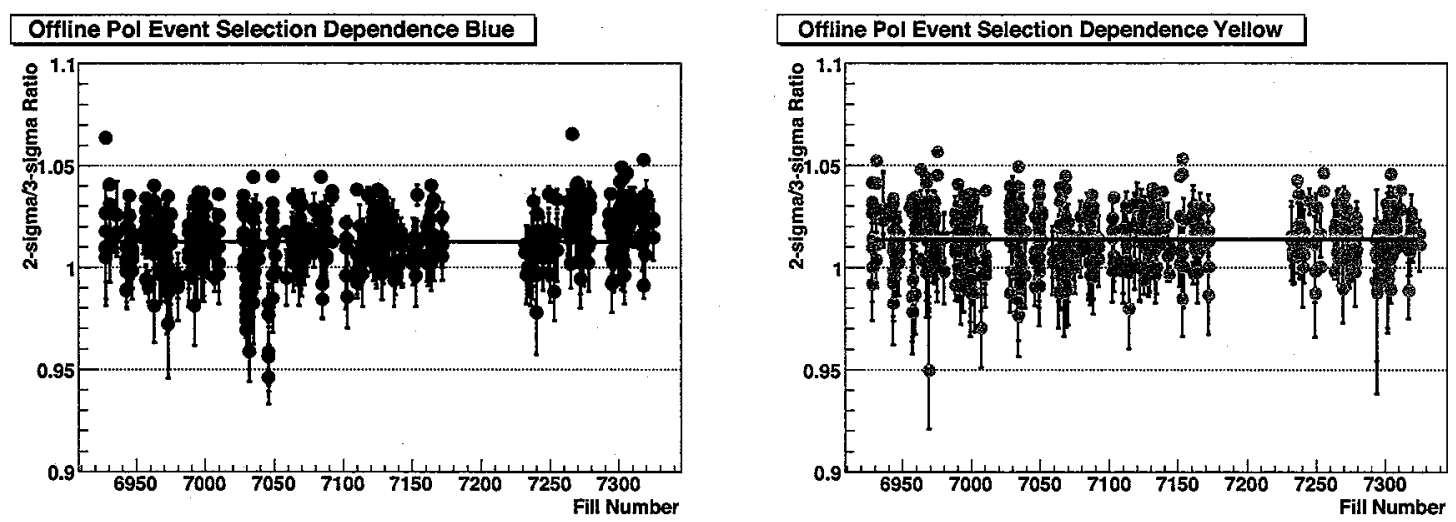

Figure 4.6: The ratio of the polarizations calculated by $2 \sigma$ and $3 \sigma$ invariant mass cuts for blue (left) and yellow (right) polarimeters. The horizontal bar shows the average. 
absolute polarization measurement by the H-Jet polarimeter. Thus we do not assign error from the background contamination to the polarizations measured by the proton-Carbon polarimeters. 


\section{Chapter 5}

\section{ANOMALY DETECTION AND QUALITY ASSURANCE}

During the physics stores of the polarized proton beam operation at RHIC, the polarization measurements were executed every two to three hours. The typical physics store lasted in 7 hours for Run05 though, regardless of the length of fill, the polarization measurements are executed right before the beam is dumped. On average, the polarization measurements are performed about 5 times per fill per beam and 20 to 25 times per day per beam. After all the polarization measurements to be analyzed in the physics store amounted more than 1300 runs throughout three monthes of running period.

It is absolutely necessary to automate the quality assurance of these data in order to extract the reliable polarizations from data within the reasonable duration. A software anomaly checker was implemented in the analysis program to detect anything behaves abnormally in the data. Major checks were categorized to following three items:

1. Energy slope

2. Strip by strip based anomaly check

3. Bunch by bunch based anomaly check

\subsection{Energy Slope Anomaly}

The inclusive cross section of the elastic proton-carbon scattering is predicted[15] to be proportional to $\exp (-60 t)$ as a function of the 4-momentum transfer. The 4-momentum transfer $-t$ is given

$$
t=-2 M E \text {. }
$$

Shown in Fig.5.1 is the exponential fit using the equation

$$
\frac{d Y}{d t}=p_{0} \exp \left(p_{1} t\right)
$$


on the energy spectrum $d Y / d t$ after the $3 \sigma$ and $400<E<900 \mathrm{keV}$ event selections. Where $p_{0}$ and $p_{1}$ are free parameters. The energy spectrum is combined for all active strips. The resulting parameter $p_{1}$ was monitored for all runs in Run 05 to assure the quality of the data.

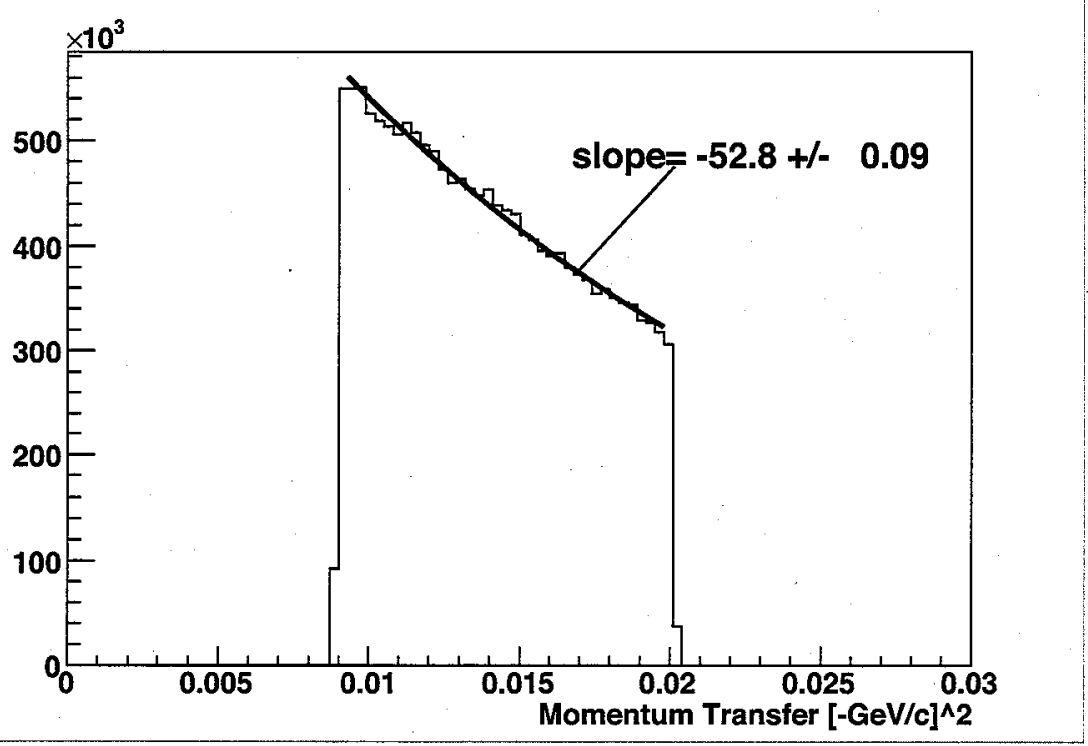

Figure 5.1: Typical energy spectrum of combined strips for all active strips. The curve is shows the best fit using the exponential function Eq.5.2.

Shown in Fig.5.2 are the parameter $p_{1}$ of the all measurements at the store energy in Run05. The slope are distributed around 50 for data points after fill number 6919 , whereas data points before 6928 distribute between -70 to -90 . This is because of the target in blue beam was longitudinally displaced to the downstream of the beam line by several $\mathrm{mm}$ $(>6 \mathrm{~mm})$. In theory the cross section of the elastic recoil Carbon beyond 90 degrees is zero. However the elastic Carbon events are still observed due to the finite acceptance of the detector plus smeared emission angle as a consequence of the multiple scattering in the target. The steeper slope than -60 suggests the latter events dominate the observed Carbon events. The target in blue beam was aligned again after fill 6919 and the polarization data using the blue polarimeter before fill 6928 were excluded from the physics analysis.

\section{$5.2 \quad$ Strip Anomaly}

There are 72 strips implemented per proton-Carbon polarimeters which were expected to function as an independent polarimeter. The consistency among these strips are one firm check can be applied. Following checks were implemented in the analysis program:

1. Number of events passed the cuts 

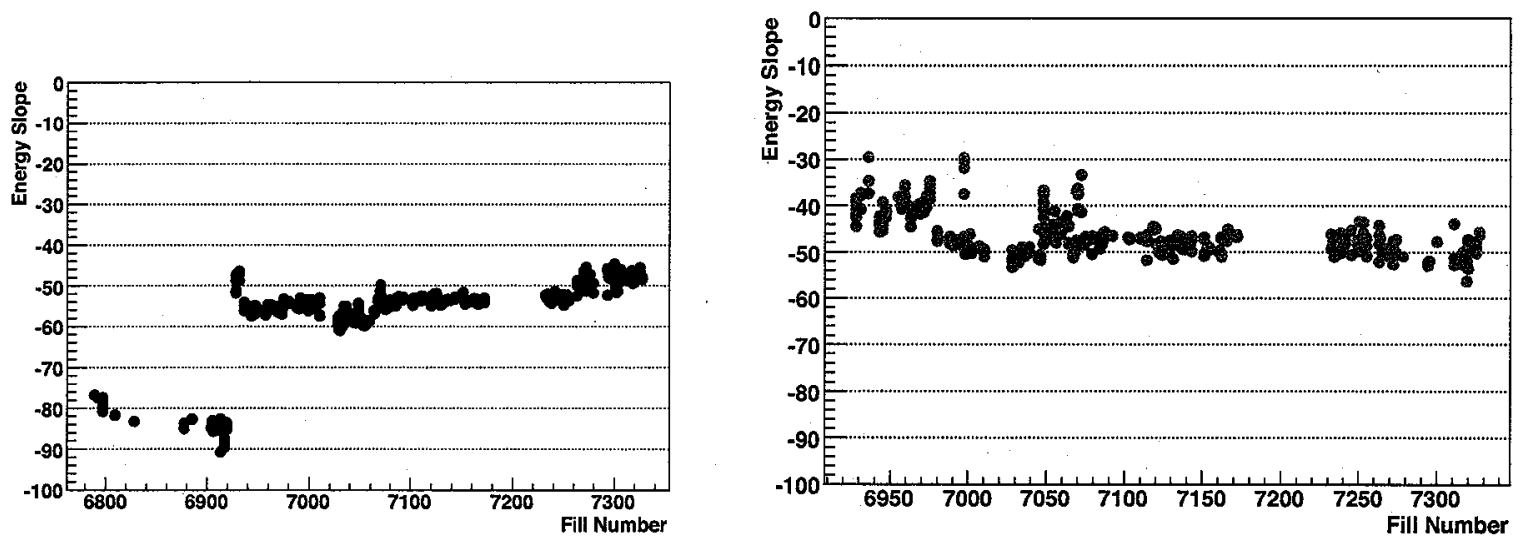

Figure 5.2: Yield slope $p_{1}$ plotted as a function of the fill number for the blue (left) and yellow (right) polarimeters.

\section{Energy to invariant mass correlation}

3. Invariant mass position

4. Invariant mass RMS width

Details are discussed in following subsections.

\subsubsection{Events Per Strip}

Shown in Fig. 5.3 are number of carbon events per strip. Event entries are for all events which passed kinematic cuts. Events outside the kinematic cuts are excluded on purpose because they are rather sensitive to the minor differences between strips in timing and the detector responses which are not well under control. Although the timing gates were set to avoid the major part of prompt events, still there are always some contaminations. Since the hardware timing gate is not designed to be tunable strip by strip, the gate timing with respect to the beam clocks are slightly different among strips due to tiny differences in cable lengths, for instance. See bottom panel of Fig. 3.10. $t_{0}$ are different between strips by a few ns between strips mostly comes from the difference in the cable lengths. As a consequence the background contaminations are slightly different strip to strip. Also the difference in the effective dead-layer thickness between strips results in visible difference in detection efficiency of low energy carbon ions. As discussed in the section energy slope anomaly, the lower the energy, the larger the cross section even exponentially. Thus number of events outside the kinematic cuts are hard to control to be consistent between strips and all strip anomaly checks discussed in this section are only based on events which passed the kinematic cuts.

The right panel of Fig. 5.3 shows number of events per strip. There seen minor acceptance structure every 12 strips. Presumably this comes from the precision limit of the mounting 
position of detectors with respect to the center of the beam pipe or the relative position of the beam orbit and the center of the beam pipe. The acceptance effect is corrected when the asymmetry is calculated. The horizontal solid and dashed lines represent the average and $+20 \%$ and $-20 \%$ boundary from the average, respectively. Any strips exceed/below $\pm 20 \%$ are flagged to grab an attention. The $\pm 20 \%$ allowance appeared to be loose enough not to pick up the acceptance effect as discussed above.

A sample of problematic run detected by the anomaly check is presented in the left panel of the Fig. 5.3. Several strips are detected to be outside the allowance. Since we have no explanation why these strips gain more or less events than others, these runs were excluded from the physics analysis.
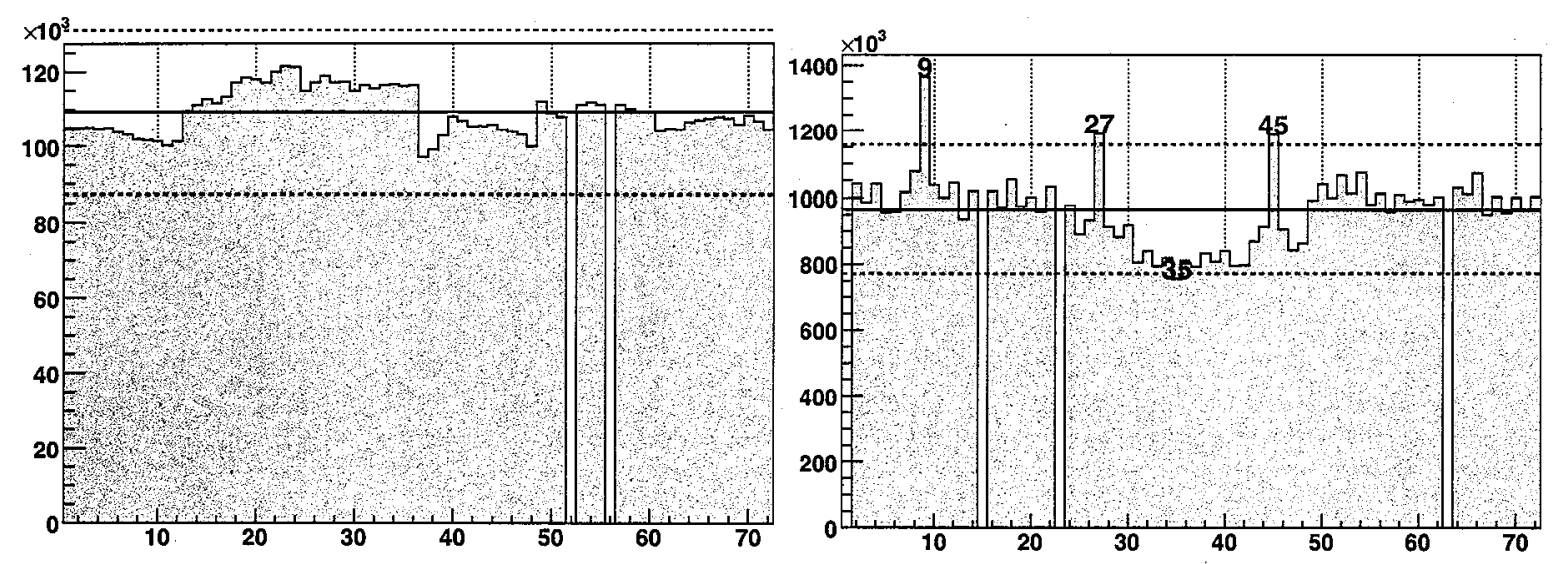

Figure 5.3: Number of events per strip of a typical good run (left) and problematic run (right).

\subsubsection{Energy to Invariant Mass Correlation}

If the kinetic energy of the recoil carbon ions are perfectly reconstructed, the shouldn't be any correlation between the reconstructed invariant mass and the kinetic energy. In reality, this principle can be broken from many reasons like the irrelevant effective dead-layer, electronics failure, and too ambitious extension of the energy range, for instance. Particularly, this is one of the way to strictly check the relevance of the effective dead-layer after the kinematics reconstruction.

Shown in Fig. 5.4 is a typical example of the energy and the invariant mass correlation plot. The data were plotted only for events which passed the kinematic cuts as described before. A linear fit was applied to the data and shown in the solid line. The fits were made for all the active strips and resulting slopes were summarized in the Fig. 5.5. The horizontal dashed lines are the $\mid$ slope $\mid<0.001 \mathrm{GeV} / \mathrm{keV}$ allowances. slope $\sim 0.001$ corresponds to $\Delta t \sim 1 \mathrm{~ns}$ or $\delta x \sim 4 \mu \mathrm{g} / \mathrm{cm} 2$. As it is discussed in the section Energy Range, the 
allowance $\mid$ slope $\mid<0.001 \mathrm{GeV} / \mathrm{keV}$ was determined in terms of our precision goal of energy reconstruction $\Delta E / E \sim 10 \%$.

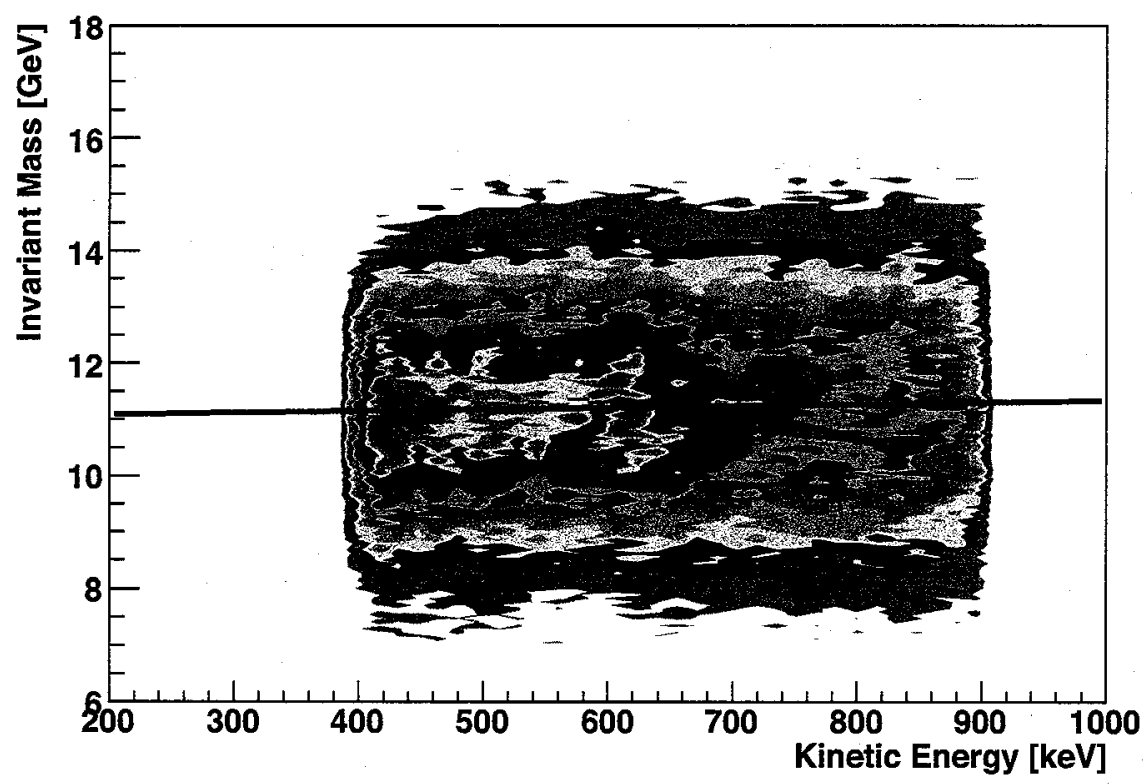

Figure 5.4: The energy to the invariant mass correlation plot. The invariant mass (vertical axis) is plotted as a function of energy (horizontal axis). The red line indicates the linear fit to the data.

The strips beyond the allowances were listed in a summary table, but they are not necessarily disabled immediately. This part of the analysis is not an automated process. The decision of disable/enabling strips were carefully processed manually referring to the summary table. Some cases the automated part of the dead-layer fit didn't work out and re-doing it by hand improve the situation. Also because of irrelevance of the present $d E / d x$ model below $E<500 \mathrm{keV}$, some strips are biased either positive or negative slope (not necessarily fluctuate around zero) and exceeds the $\mid$ slope $\mid<0.001 \mathrm{GeV} / \mathrm{keV}$ allowance border from time to time even the magnitude of fluctuation is rather minor as shown in Fig. 5.6. There are a couple strips per blue/yellow polarimeters behaved like this and they were not disabled from following two reasons 1) the deviation from the allowance is small because it is driven within the normal magnitude of the fluctuations, 2) even if we allocate the systematic error to save these strips, the impact on the total error is still negligibly small because it is still one of over 70 active strips.

Typical cases which results in disabling strips are relatively major break of the $\mid$ slope $\mid<$ $0.001 \mathrm{GeV} / \mathrm{keV}$ as shown in the right panel of Fig. 5.7. There observed the major excursion between the fill 7029.102 and 7049.103. Such a major break was mostly driven by electronics failures or just poor statistics. The excursion of strip number 32 was driven by the former and was disabled entirely this period despite the slopes get within allowance for some data points. The phenomena of this electronics failure is discussed more details in the next section. 


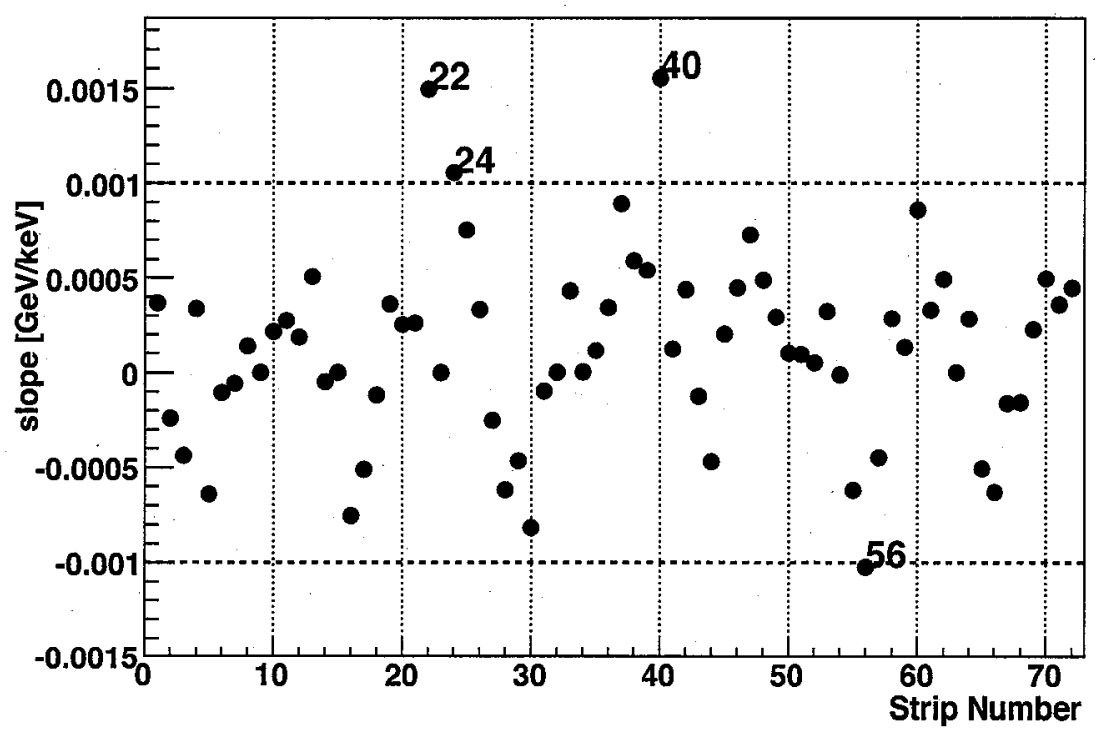

Figure 5.5: The slope distribution of the energy to the invariant mass correlation. The horizontal axis is the strip number. Red dashed line is the $\mid$ slope $\mid<0.001$ allowance.

Thus one of the anomaly is somehow quantified to be detectable by the analysis software, nevertheless the amount of information is still painfully too many, imagine monitoring 72 strips for 1300 runs. An useful reference to effectively minimize the required attention is to focus on the maximum slope among all active strips for each run. Shown in Fig. 5.8 is the the maximum slope of each runs plotted as a function of the fill number.

As described above, major deviations from the border are likely be caused by the electronics failures. After applying above operations discussed so far, Fig.5.9 are finally obtained. There are still exceeding $\mid$ slope $\mid<0.001 \mathrm{GeV} / \mathrm{keV}$ allowances data points, but we didn't disable any more strips because number of strips which flagged to be anomaly are already a few (see Fig.5.10 and the impact to the total systematic error is in the negligible level. The key point is to surely remove the strips which deviates significantly from the normal behavior and therefore can impact on the resulting polarization non-negligibly. The flagged strips in Fig.5.10 are mostly dominated by the mass-energy correlation for the yellow polarimeter though, the width of the invariant mass also makes a significant contribution for the blue polarimeter as shown in Fig. 5.11. The width of the invariant mass will be discussed in the next section in details. The anomaly strip statistics shown in Fig. 5.10 and Fig. 5.11 are the final status of the Run05 analysis. Again we didn't tried to fix or disable all the strips which are detected by the anomaly detectors, because some of them are minor and barely impact on the resulting polarizations. 

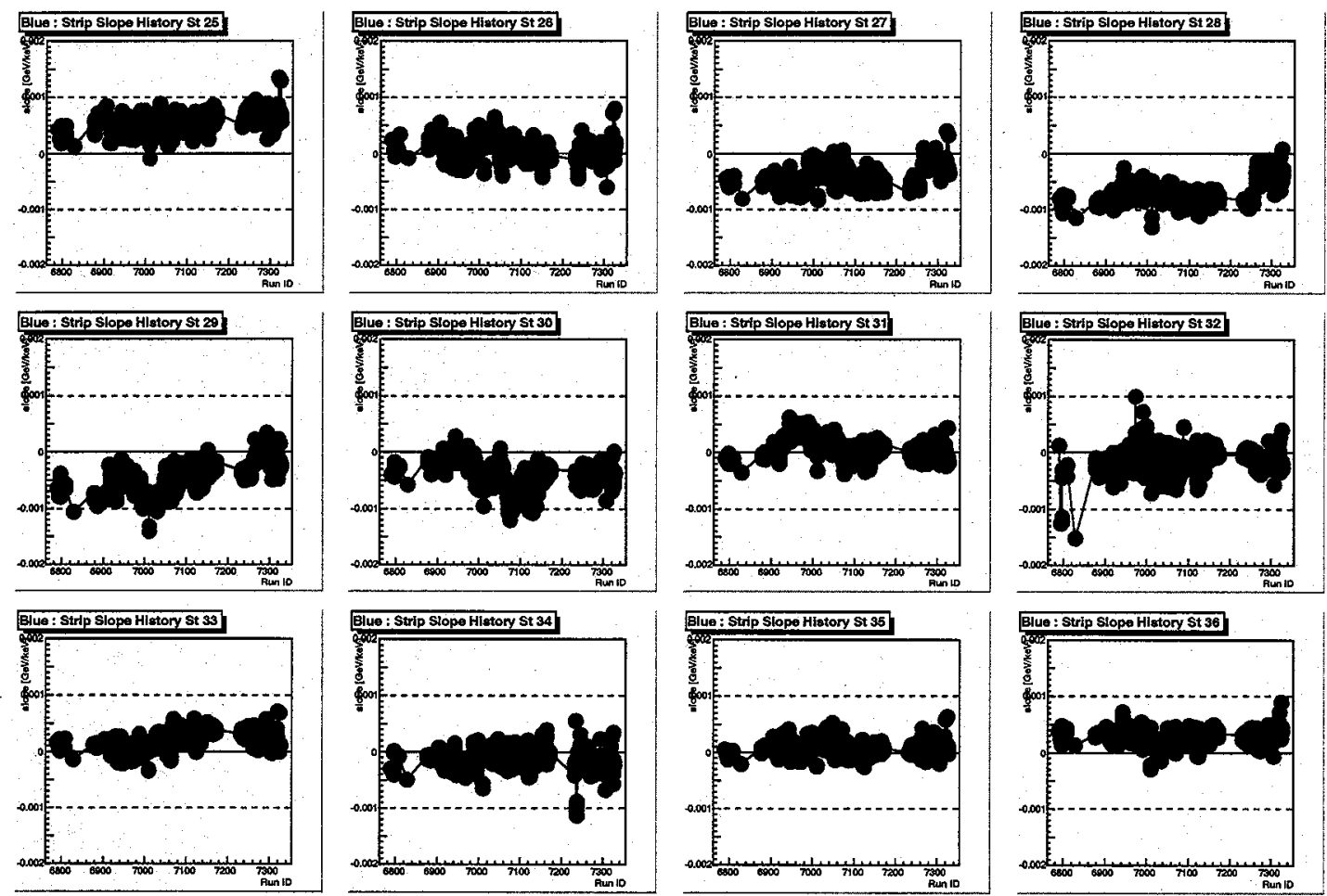

Figure 5.6: The energy and the invariant mass correlation slope history for the strip number $25-36$ of the blue polarimeters. The horizontal axis shows the fill number and the dashed lines represent slope $= \pm 0.001 \mathrm{GeV} / \mathrm{keV}$ allowance borders.

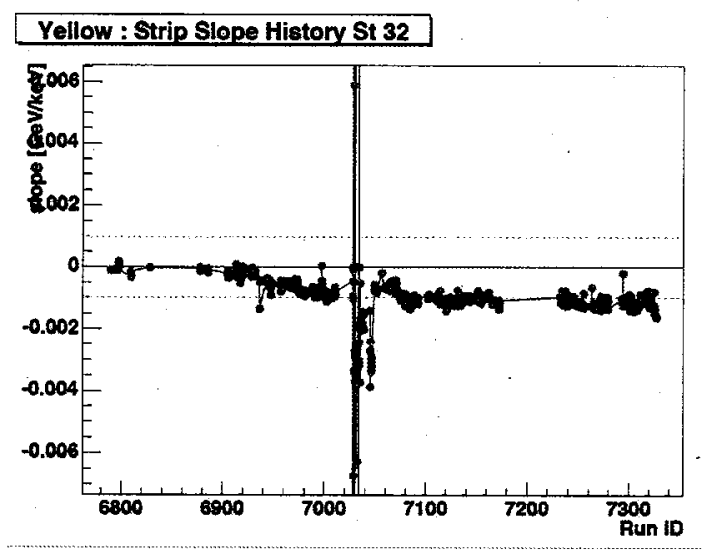

Figure 5.7: The energy and the invariant mass correlation slope history for the strip number 32 of the yellow polarimeter. 

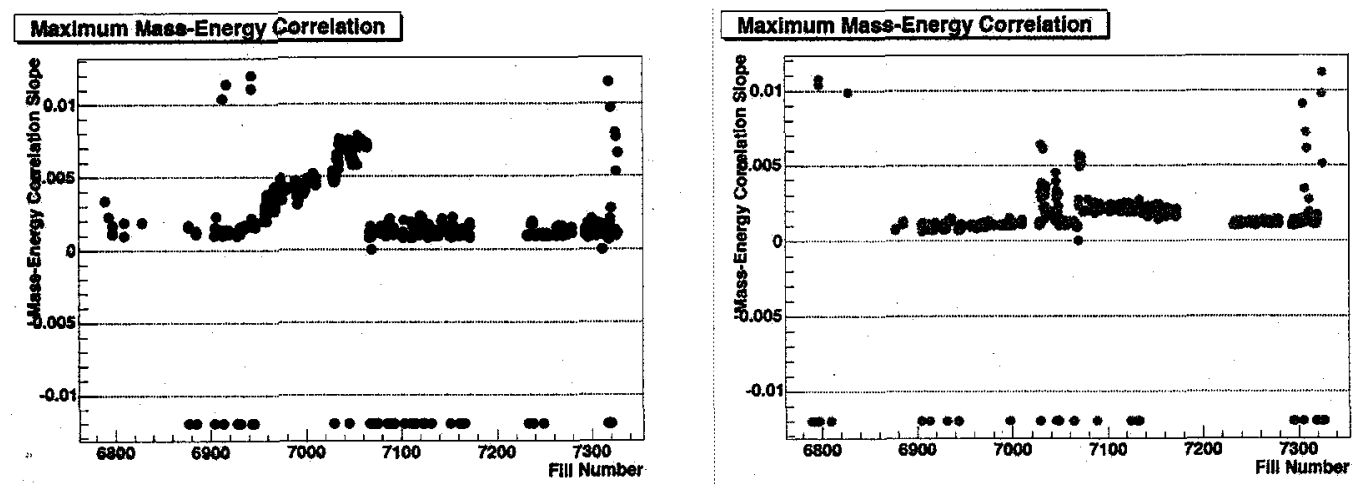

Figure 5.8: The maximum deviation of the energy and the invariant mass correlation slope for all active strips plotted for all measurements in physics stores. Left and right panels are results of the detectors for the blue and yellow polarimeters, respectively.
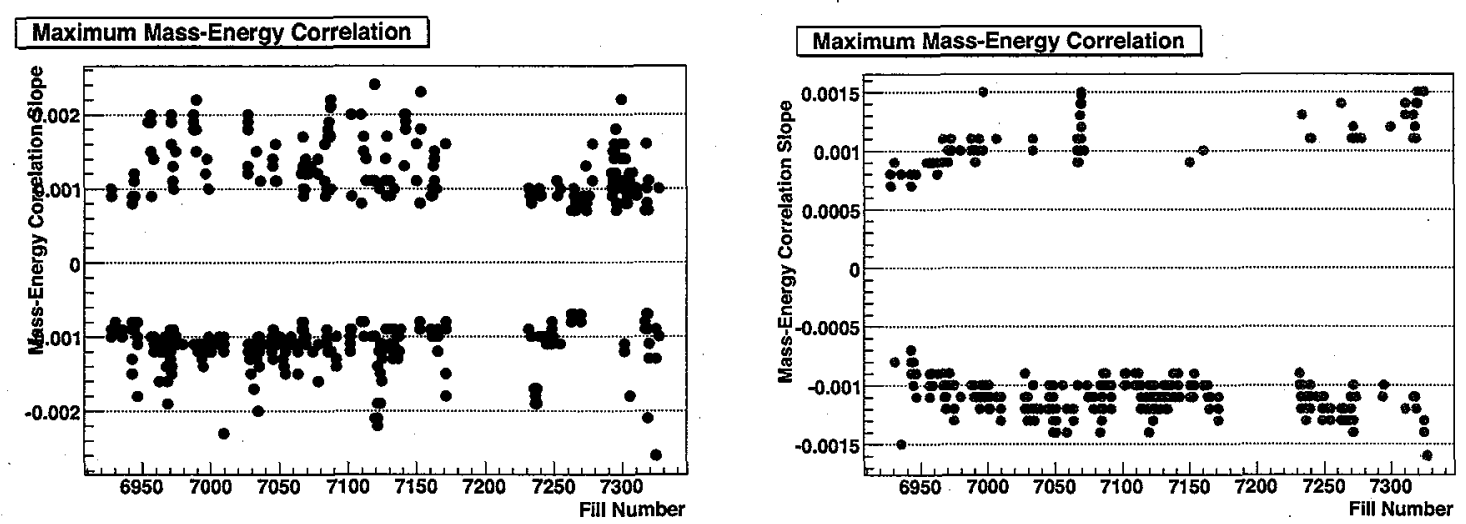

Figure 5.9: Same as Fig.5.8, but after re-evaluating the effective dead-layer or disabling strips: 

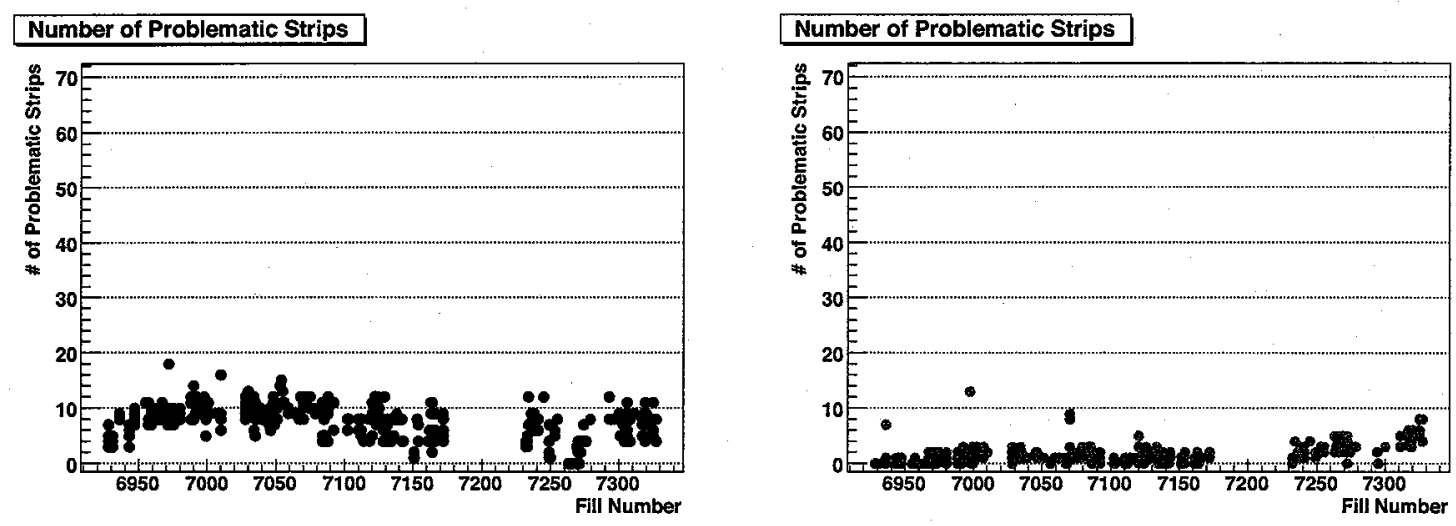

Figure 5.10: Number of strips flagged by the anomaly detectors for blue (left) and yellow (right) polarimeters. The anomaly detection includes not only the energy-invariant mass correlation but also 3 other strip checks:
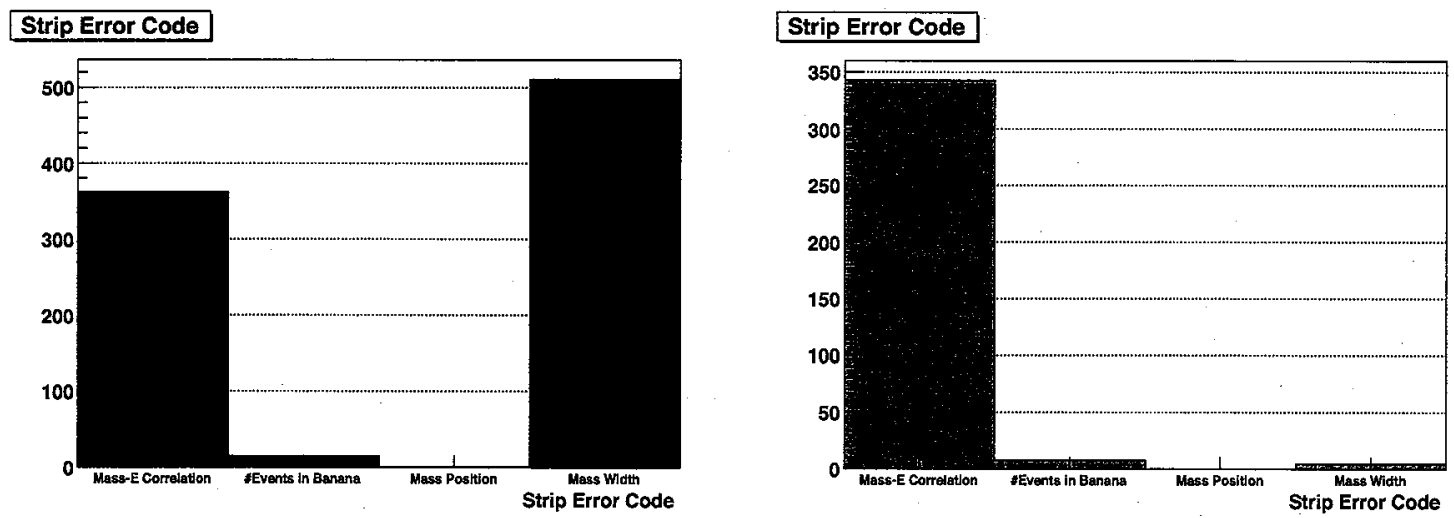

Figure 5.11: Incremented strips errors for the 4 anomaly check items for the all data in physics stores. A single strip of a single run can make a multiple entries. 


\subsubsection{Invariant Mass Position and RMS Width}

The remaining two anomaly checks were based on the Gaussian fitting results on the reconstructed invariant mass of Carbon. For this particular purpose, the spectrum to be fitted does not contain any events out side of the kinematic cuts as seen in Fig.5.12. The both wings to the side were cut off outside $3 \sigma$ of width.

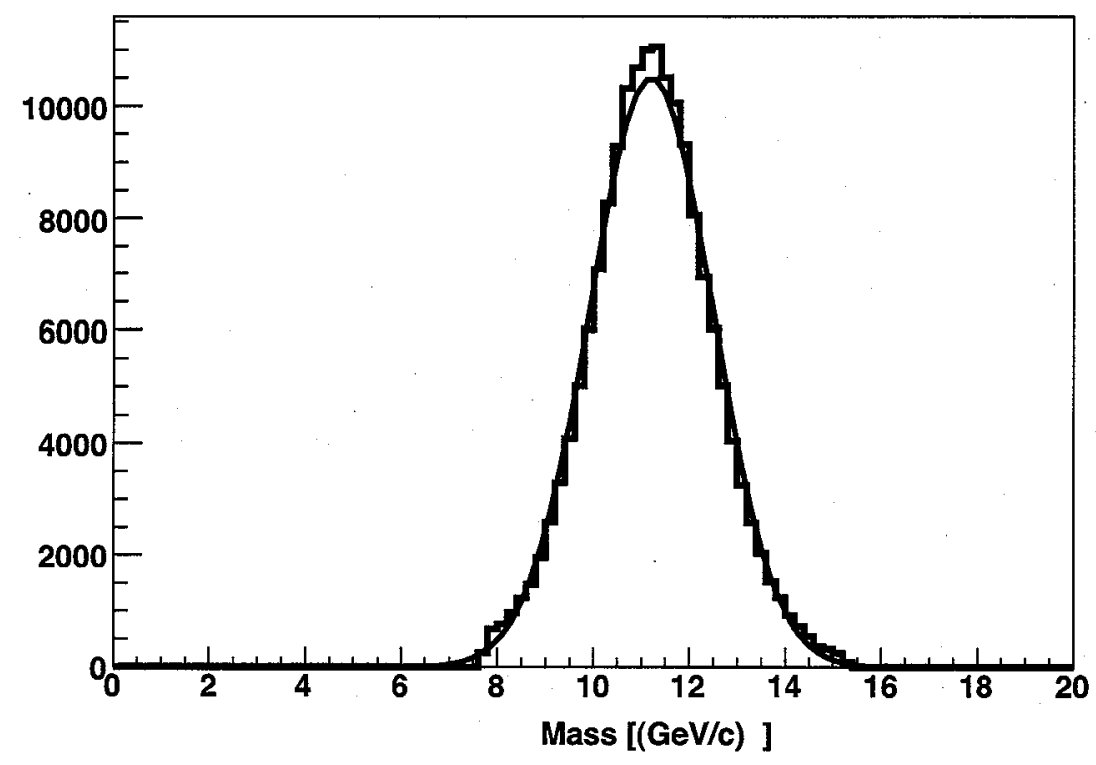

Figure 5.12: A Gaussian fit applied to a reconstructed invariant mass.

Shown in Fig. 5.13 is a typical mass peak position distribution plotted as a function of the strip number. In principle, the dead-layer fit for each strip should bring the invariant mass peak to be at exactly Carbon mass though, the actual data may not necessarily appear at the exact location for following two additional steps: 1) as it discussed in section 3.2, the effective dead-layers was averaged over the detector and treated as a fixed parameter in the next fitting round. The $\chi^{2}$ of next $t_{0}$ single parameter fit won't be as good as the first two parameter fit for the strips whose best fit effective dead-layer is far away from the average. 2) As discussed in the section Event Reconstruction, the $t_{0}$ adjustment at the beginning of the kinematics reconstruction program is done with sparcified events by 100 and therefore the precision of $t_{0}$ deteriorates drastically when statistics are poor.

The strips beyond the deviation of the mass position $\Delta M>0.5 \mathrm{GeV}$ are again flagged as anomaly in the strip.

Shown in the Fig. 5.14 is worst deviation among the active strips in the invariant peak position from the Carbon mass plotted as a function of the fill number for blue (left) and yellow (right) polarimeters after fixing/disabling strips $\Delta M>0.5 \mathrm{GeV}$. All data points are 


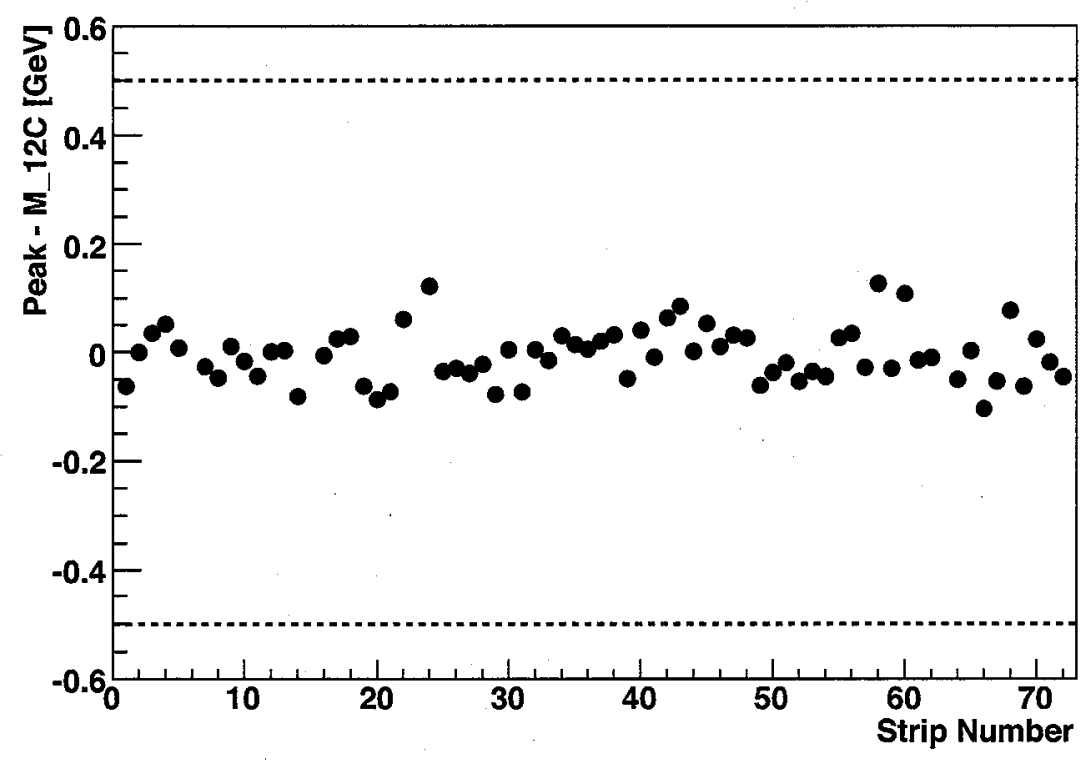

Figure 5.13: The deviations of the reconstructed invariant mass position from the carbon mass are plotted as a function of strip number.

below $\Delta M<0.5 \mathrm{GeV}$ and are considered as a satisfactory. Note this is the worst deviation which means the rest of the strips are in better agreement.

Similar checks are also attempted to the RMS width of the invariant carbon mass peaks, but the reference width is given by the strip average which is calculated run-by-run basis unlike the peak position, because the RMS width is not invariant quantity. The width of the invariant mass are determined by multiple components. Major ones in the present system are follows:

1. Energy resolution of the polarimeter

2. Time resolution of the polarimeter

3. Longitudinal beam bunch profile

Typically the RMS widths are between 1 to $1.5 \mathrm{GeV}$. Shown in Fig.5.15 is the typical mass RMS width $\sigma^{\text {RMS }}$ distributions plotted as a function of the strip number. Left panel is the example with no anomaly detection whereas left panel with some anomaly detections. Several strips exceeds the $0.1 \mathrm{GeV}$ allowance limit from the average width. $0.1 \mathrm{GeV}$ allowance limit was selected empirically to detect these anomalies with a good efficiency. In principle, the RMS width are quite consistent among strips within way below $0.1 \mathrm{GeV}$ under the normal circumstances. 

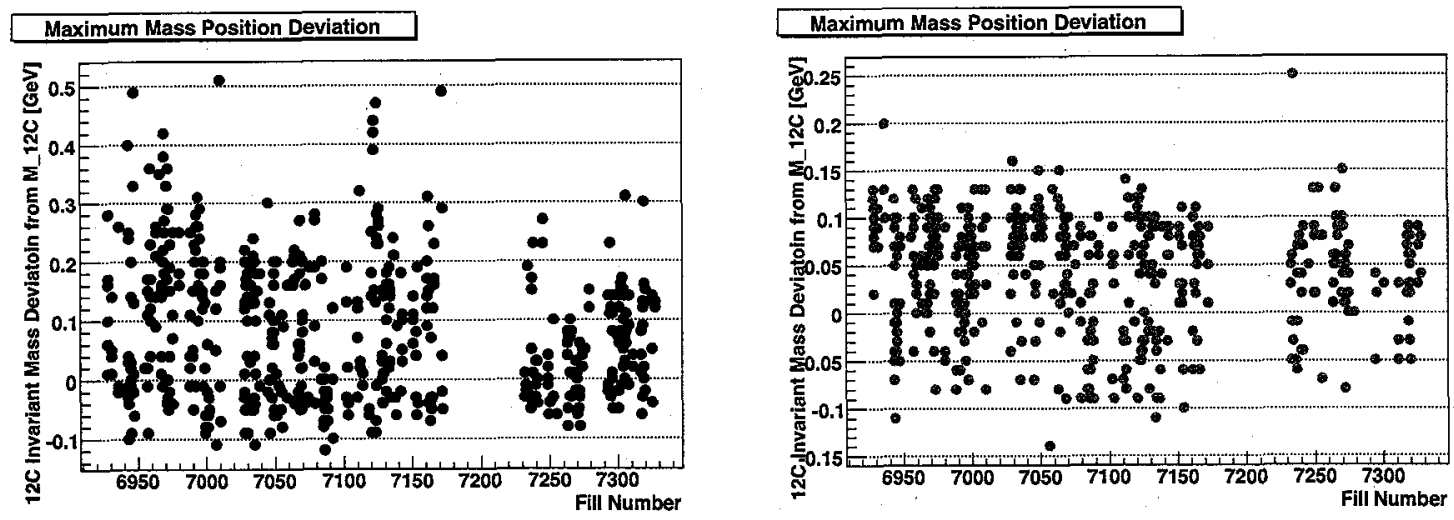

Figure 5.14: The worst deviation among all active strips in the invariant peak position from the Carbon mass plotted as a function of the fill number for blue (left) and yellow (right) polarimeters after fixing/disabling strips $\Delta M>0.5 \mathrm{GeV}$.
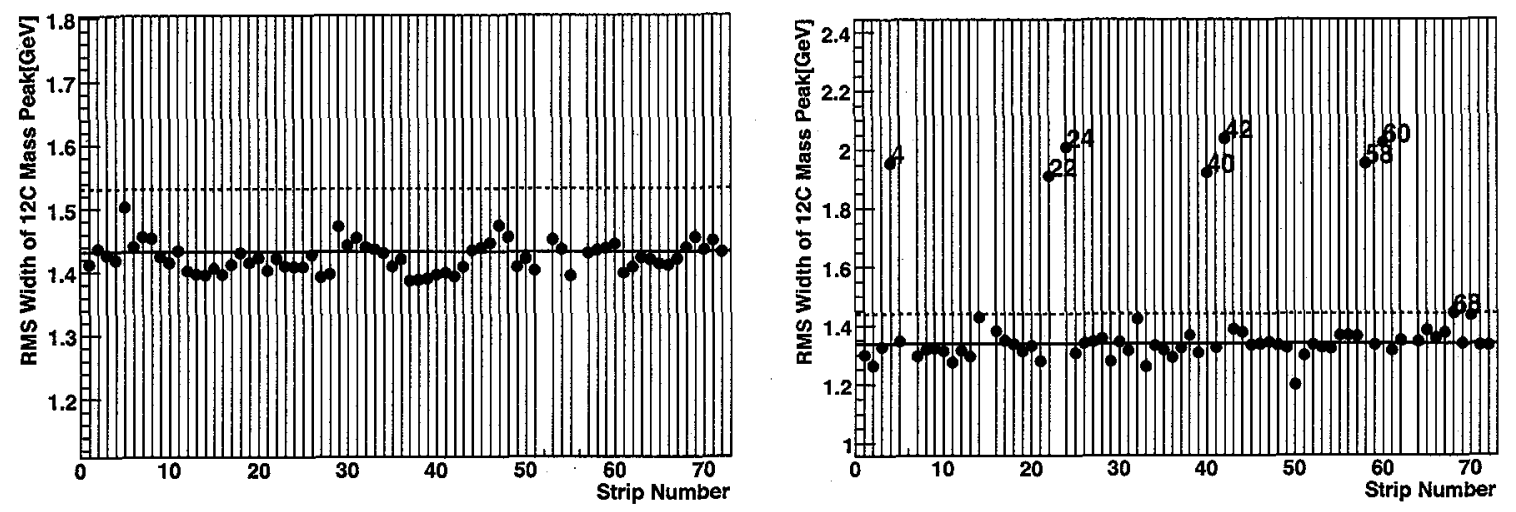

Figure 5.15: A typical mass RMS width distributions plotted as a function of the strip number. Left panel shows no anomaly whereas the right panel detected bad strips which exceeds the allowance limit $+0.1 \mathrm{GeV}$ (dashed line) from the weighted average (solid line). 
Since the anomaly is detected with respect to the reference, the average needs to be calculated in a reliable manner. When the anomalies are occurred as shown in Fig. 5.15, a simple average without any weight would be heavily biased by the anomaly strips which are much wider than normal width. Since this is the whole purpose to detect the abnormal strips using the deviation from the average which includes the abnormal RMS width.

Shown in Fig. 5.16 is the typical time-of-flight to energy correlation plot (left) and the invariant mass distribution (right) detected as anomaly. As can be seen from the left figure, there observed double bands of the elastic carbon events in the middle of the plot. This double bands are likely to be caused by a timing jitter of the beam clock either within the WFD module or at the output of the fan out module which distributes the beam clock to all WFD modules. Since the strips every 18 channels are sharing the same WFD module, 4 strips goes anomaly once this jitter happens. As a consequence of two distinct different timing, the reconstructed invariant mass distribution appears to have double peaks as shown in the right panel.
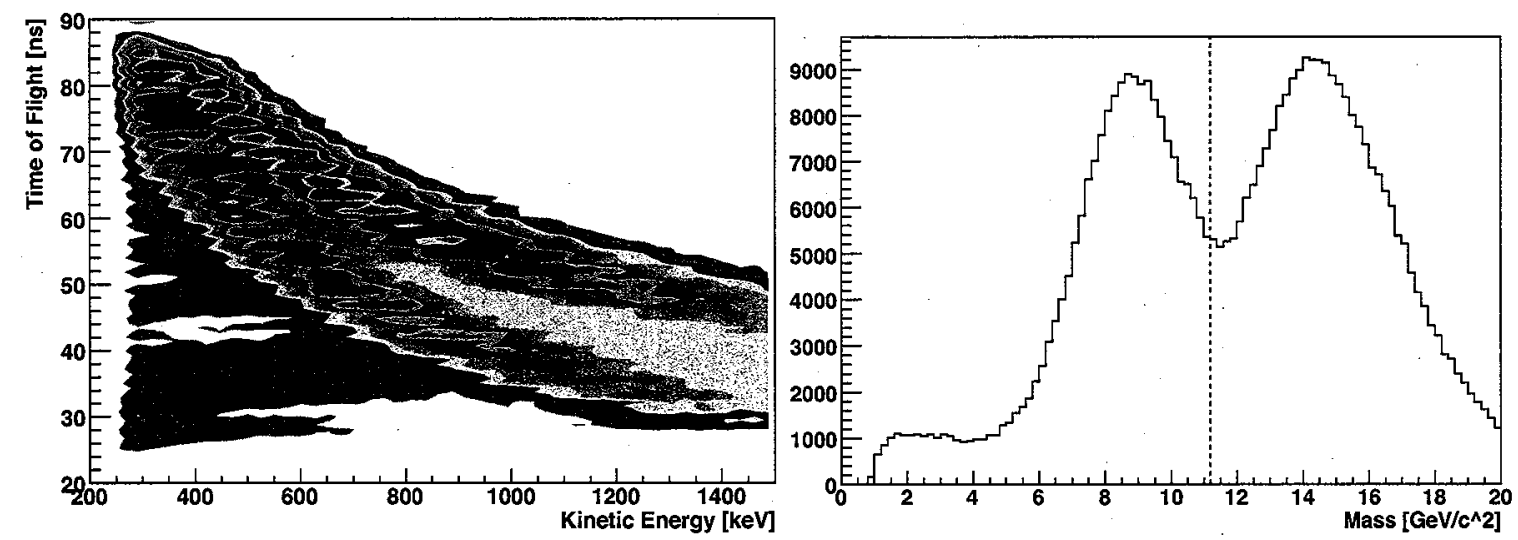

Figure 5.16: The time-of-flight to energy correlation plot (left) and the invariant mass distribution (right) detected as anomaly. The elastic carbon events split into double bands in the correlation plot and resulted in double peaks in the reconstructed invariant mass.

Throughout Run05 analysis these extraordinary wider RMS width in the invariant mass were caused by the timing jitter problem. In such a case, the resulting $\chi^{2}$ is large because the experimental invariant mass spectrum is split into two peaks whereas the fitting function is a single Gaussian. The average RMS $\left(\overline{\sigma^{\mathrm{RMS}}}\right)$ is thus weighted by $\left(\chi^{2}\right)^{2}$ :

$$
\overline{\sigma^{\mathrm{RMS}}}=\frac{\sum_{s t} \frac{\sigma_{s t s}^{\mathrm{RMS}}}{\Delta \sigma_{s t}^{\mathrm{RAS}}\left(\chi_{s t}^{2}\right)^{2}}}{\sum_{s t} \frac{1}{\Delta \sigma_{s t}^{\mathrm{RMS}}\left(\chi_{s t}^{2}\right)^{2}}}
$$

where $i$ runs for all active strips. The weight $\left(\chi^{2}\right)^{2}$ has employed through empirical try and error to detect the anomalous strips most efficiently. The time jitter problem occurred quite frequently during Run05, where the the magnitude of jitter varied between 1 to 3 
ns. Since the event selection is applied based on $\sigma$, the jitter in timing wouldn't affect on the polarization for the first order. Of cause the wider the $\sigma$, the more background events contaminate within the event selection cuts, but this is relatively minor effect. Therefore strips were disabled only when the magnitude of jitter were remarkable.

\subsection{Bunch Anomaly}

As discussed in the section 3.3.3, the beam in RHIC has bunch structures at the maximum filled bunches of 112. Each beam in bunches are polarized either positive or negative following prefixed spin patterns. The magnitude of the polarization supposed to be consistent from one bunch to another bunch. There is no customized mechanism to polarize different magnitudes bunch by bunch in RHIC. Thus the consistency of the polarization from one bunch to another is another good systematic check of the polarimeter. Since every accumulated single events are identified by the belonging bunch ID in the data stream, it is possible to evaluate asymmetries bunch by bunch basis.

The asymmetry for a given bunch bid is defined as follows:

$$
A_{b i d}=\frac{N_{b i d}^{\mathrm{L}}-R_{b i d}^{\mathrm{lumi}} N_{b i d}^{\mathrm{R}}}{N_{b i d}^{\mathrm{L}}+R_{b i d}^{\text {lumi }} N_{b i d}^{\mathrm{R}}}
$$

where bid runs for the bunch ID, $N_{b i d}^{\mathrm{L}}\left(N_{b i d}^{\mathrm{R}}\right)$ is the number of events (after event selection cuts) in the left (right) detector of the bunch $\mathrm{ID}=k$. The luminosity of the bunch bid is calculated ${ }^{1}$ by taking sum of $N_{l}^{\mathrm{L}}$ and $N_{l}^{\mathrm{R}}$ ( $l$ runs for bunch up to 112) excluding events in own bunch $N_{b i d}^{\mathrm{L}}$ and $N_{b i d}^{\mathrm{R}}$ and unfilled bunches, respectively.

$$
R_{b i d}^{\mathrm{lumi}}=\frac{\sum_{l} N_{l}^{\mathrm{L}}}{\sum_{l} N_{l}^{\mathrm{R}}}
$$

In principle, the physical asymmetry of vertically polarized beam can be measured by taking the left-right combinations using detectors mounted at azimuthal angle of 90 degrees $(\mathrm{D} 2, \mathrm{D} 4)$ as shown in the top-left panel in Fig. 5.17. This combination is named X90 combination. Using detectors combination mounted at 45, 135, 225 and 315 degrees, the physics asymmetry can be also measured as shown as a X45 combination in the top-right panel in Fig. 5.17. The independent measurement of the raw asymmetry is quite important to monitor the systematics of the detection system. Although the sensitivity of X45 combination to the vertical polarization is suppressed by $\sqrt{2}$ than that of X90, statistical abundance compensates it by combining events in four detectors (D1+D3,D4+D5). Taking advantage

\footnotetext{
${ }^{1}$ The current version of the program BunchAsymmetry in AsymCalc.cc (ver.1.76) do not exclude own bunch in the luminosity calculation. This needs to be modified to be

$$
R_{b i d}^{\text {lumi }}=\frac{\sum_{l \neq b i d} N_{l}^{\mathrm{L}}}{\sum_{l \neq b i d} N_{l}^{\mathrm{R}}}
$$


of the possible combinations can be made out of 6 detectors, forbidden asymmetries with respect to the vertical spin vector can be evaluated. For instance, finite asymmetry should be observed by the combinations of $(\mathrm{D} 1+\mathrm{D} 5, \mathrm{D} 3+\mathrm{D} 4)$ only when the spin vector has a radial component as shown in the bottom-left panel. In other word, the Y45 combination supposed to be zero when the spin vector is pointing perfectly vertical. As shown in the bottom-right panel, the observation of the finite asymmetry in the cross combination (D1+D4,D3+D5) immediately indicates systematic anomaly in a measurement.

Physics Asymmetry
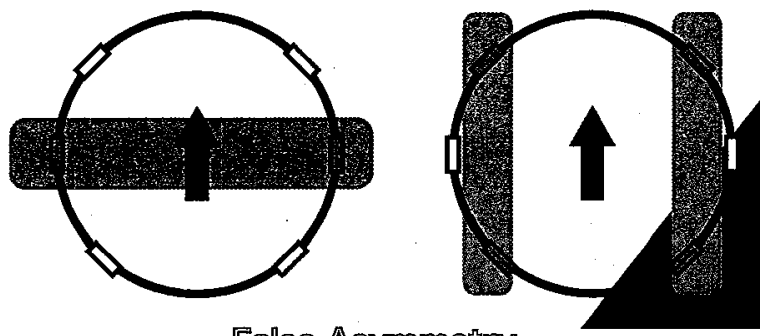

Fallse Asymmetry
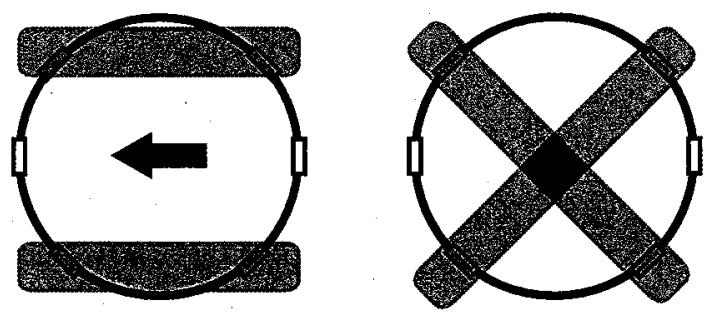

Figure 5.17: Detector combinations which form physics (top) and false (bottom) asymmetries.

Shown in the Fig. 5.18 are the raw asymmetries of X90 (top left), X45 (top right), and Y45 (bottom left) plotted as a function of bunch number. Red and blue solid circles represent positively and negatively polarized bunches based on the polarization pattern information distributed from the accelerator. In both physics asymmetries X90 and X45, red and blue solid circles are well consistent to each other and indicates finite asymmetries. On the contrary, blue and red circles are distributing around zero in Y45 asymmetries. In this typical good example, all asymmetry combinations show $\chi^{2}$ of around 1 to the mean for both positive(+) and negative(-) bunches as printed in the top and bottom corners of asymmetry plots in Fig. 5.18.

The mean of the raw asymmetries are calculated by the Gaussian fits to the asymmetry distributions as shown in Fig. 5.19. Each entry to the histogram is the raw bunch asymmetry of X90 combination weighted by the statistics. Since the maximum entry is limited to 112 , the raw asymmetries of the negatively polarized bunches are flipped sign and combined with those of positively ones assuming symmetries between them. The mean for the negatively 

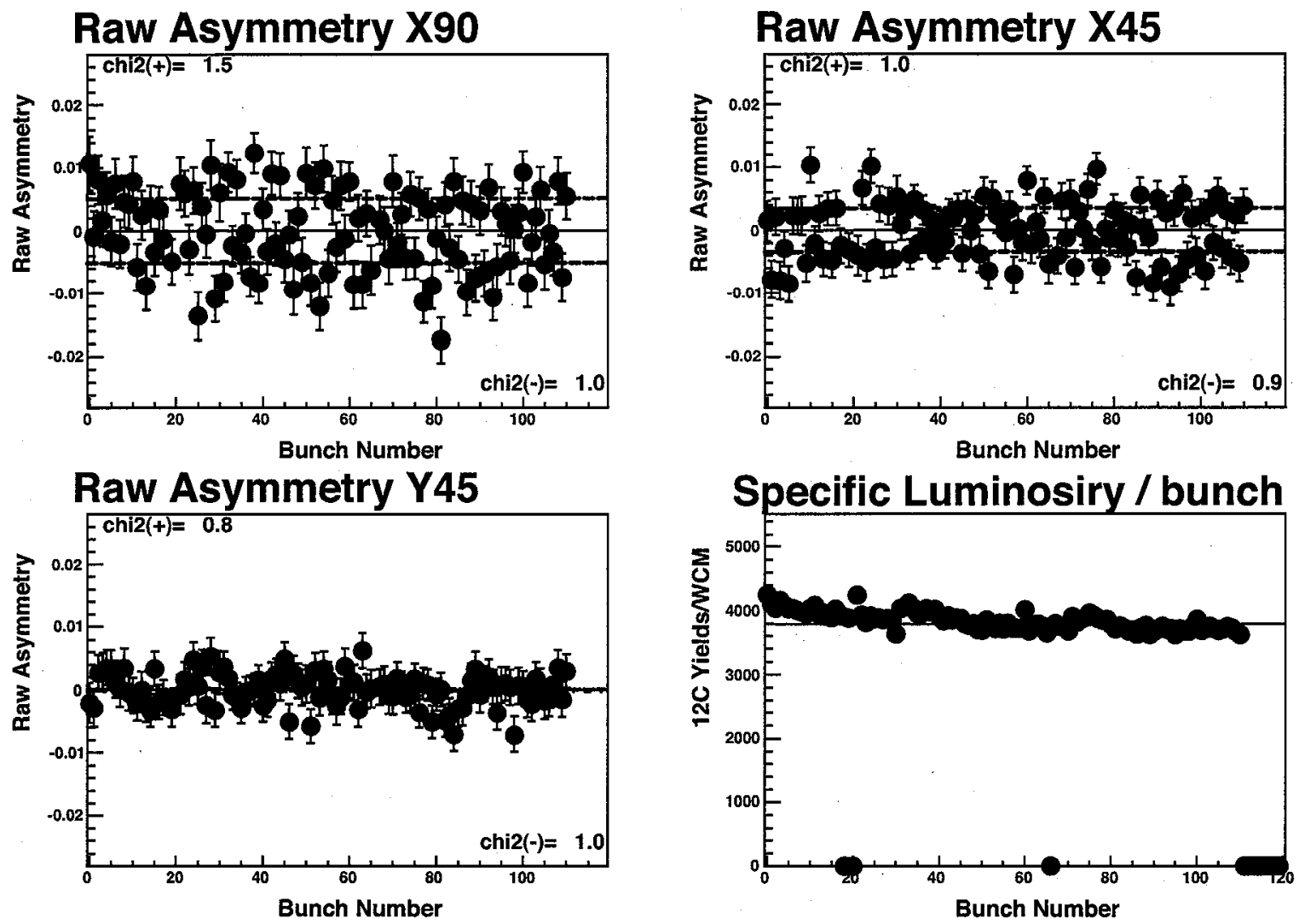

Figure 5.18: Typical example of bunch asymmetries for three different combinations of detectors, i.e. X90 (top left), X45 (top right) and Y45 (bottom left). Red and blue solid circles represent raw asymmetries of positively and negatively polarized bunches, respectively. The $\chi^{2}$ with respect to the mean (dashed lines) are printed at the top and bottom corners of these panels. The bottom-right panel shows the specific luminosity distribution as a function of bunch number. 
polarized bunches are thus obtained by flipping sign of the resulting mean of the Gaussian fit. Means for other combinations i.e. X45 and Y45 are calculated similarly.

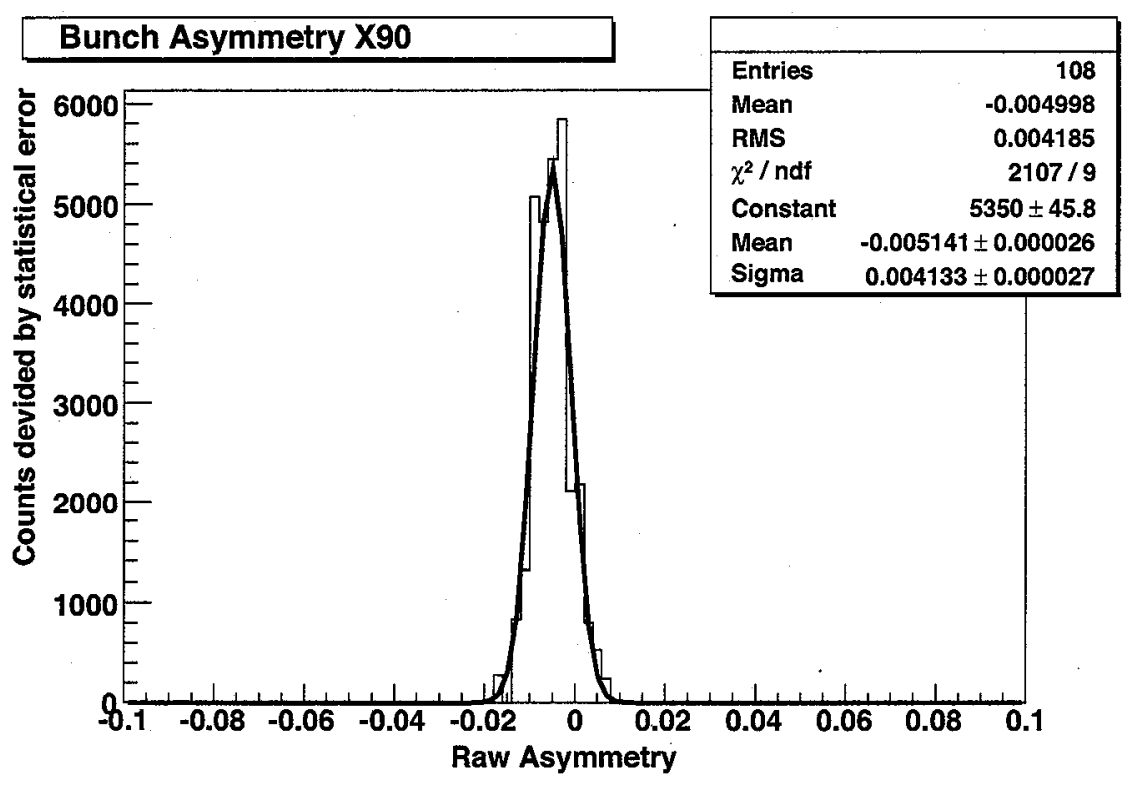

Figure 5.19: A Gaussian fit (red curve) on the bunch asymmetry distribution for the typical raw asymmetry of the X90 combination.

The bottom right panel in the Fig. 5.18 is the specific luminosity distribution for each bunches. Specific luminosity is defined by the ratio between number of events per bunch within kinematic cuts and the intensity per bunch measured by the wall current monitor[16]. The number of events are summed for all active strips for a given bunch. The number of events accumulated by the polarimeter detectors are proportional to the beam intensity and the beam emittance, whereas the wall current monitor is not sensitive to the emittance. Thus any structures seen in the bunch distribution of the specific luminosity are likely originated from the difference in the emittance from a bunch to another.

Shown in Fig. 5.20 is the one of the measurement which was flagged by the bunch anomaly detector. The asymmetry anomaly detector was programmed to flag any bunches which deviates greater than $5 \sigma$ from the mean of the asymmetry distribution. Shown in Fig. 5.20 is the bunch by bunch asymmetry histogram of the X90 combination, which is a projection of Fig. 5.20 to the vertical axis and each entry to the histogram was weighted by the statistical error. All negatively polarized bunches are flipped its asymmetry sign. A Gaussian fit was applied to the histogram in order to determine the mean. The bunches flagged for significant deviation from the mean is defined by $5 \sigma$ away from the mean, where $\sigma$ is the statistical error of the given bunch, not the width of the Gaussian distribution. The spread of the asymmetry distribution is not considered in the asymmetry anomaly detection.

As listed in the Table. 5.1 and Table. 5.2, there found anomalies in bunch asymmetries in 

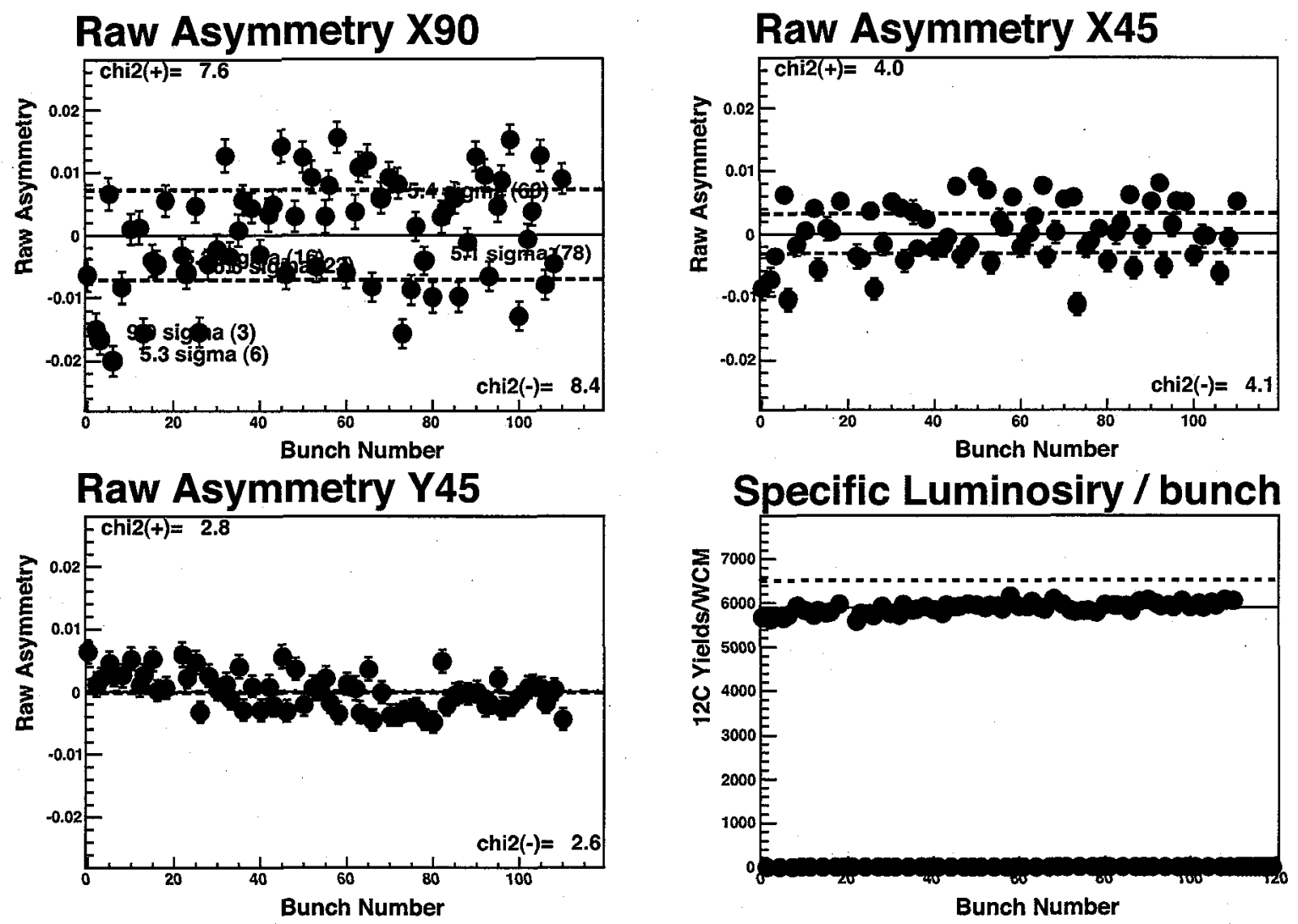

Figure 5.20: An anomaly found in the yellow polarimeter during fill 7048. The anomaly detector found several bunches deviates more than $5 \sigma$ away from the averages in the X90 asymmetry combination. These anomaly bunches are highlighted in the plot with the magnitude of the deviation in the unit of $\sigma$ and the strip number in a bracket. 
several fills. Most of the cases, the cause of the bunch anomalies are not identified. We don't even know. whether they are originated from the beam or the polarimeter system. If we are able to eliminate the possibility of the latter case, then the observed unstable behavior of the bunch asymmetry becomes quite likely to be real.

Unlike the most of cases detected by the asymmetry anomaly detector, the source of the suspicious behavior of bunch asymmetries observed in the fill 7048 is one of few cases that the cause was identified. The anomaly in the bunch asymmetries were all the sudden observed at the beginning of fill 7048 in the yellow polarimeter as soon as the target was changed to thicker one $e^{2}$. The raw event rate (no event selection cut, combined all strips) was jumped up to more than $6 \mathrm{MHz}$ which is more than factor of two higher than the measurements in previous fill 7046 (around $2.8 \mathrm{MHz}$ ). The pure rate increase by the target change can be roughly calculated by the beam intensity correction (average of the WCM of all filled bunches: $87 \times 10^{9}$ protons for 7048.104 and $80 \times 10^{9}$ protons for 7046.104$): \frac{6}{2.8} \cdot \frac{80 \times 10^{9}}{87 \times 10^{9}} \approx 2$. Not only the asymmetry, we also observed extraordinary higher background contaminations in the data as shown in the invariant mass spectra, Fig.5.21. The fraction of the background events in the total entry is relatively higher than the typical invariant mass shown in Fig.4.5.

Table 5.1: The list of blue polarimeter runs/fills which were detected as anomaly in the bunch asymmetries by the anomaly detector.

\begin{tabular}{ccc}
\hline \hline Run/Fill & Anomaly & Cause \\
\hline 7293.002 & Bunch ID $<20$ & unknown \\
7327.004 & Bunch ID $<20$ & unknown \\
\hline \hline
\end{tabular}

Table 5.2: The list of yellow polarimeter runs/fills which were detected as anomaly in the bunch asymmetries by the anomaly detector.

\begin{tabular}{ccc}
\hline \hline Run/Fill & Anomaly & Cause \\
\hline 7048 & Diverged bunches & Thick target \\
7053 & $70<$ Bunch ID $<80$ & unknown \\
7054 & $70<$ Bunch ID $<80$ & unknown \\
7055 & $70<$ Bunch ID $<80$ & unknown \\
7056 & $70<$ Bunch ID $<80$ & unknown \\
7325.102 & Bunch ID $<20$ & unknown \\
7327 & Bunch ID $<20$ & unknown \\
\hline \hline
\end{tabular}

\footnotetext{
${ }^{2}$ The thickness of the Target-3 (used before the fill 7048) was $17.8 \mu \mathrm{g} / \mathrm{cm}^{2}$, while the Target-1 (used during the fill 7048) was tapered, i.e. $13.8 \mu \mathrm{g} / \mathrm{cm}^{2}$ at the edge and $42.7 \mu \mathrm{g} / \mathrm{cm}^{2}$ at the center. We don't know where the beam hit on the target body
} 

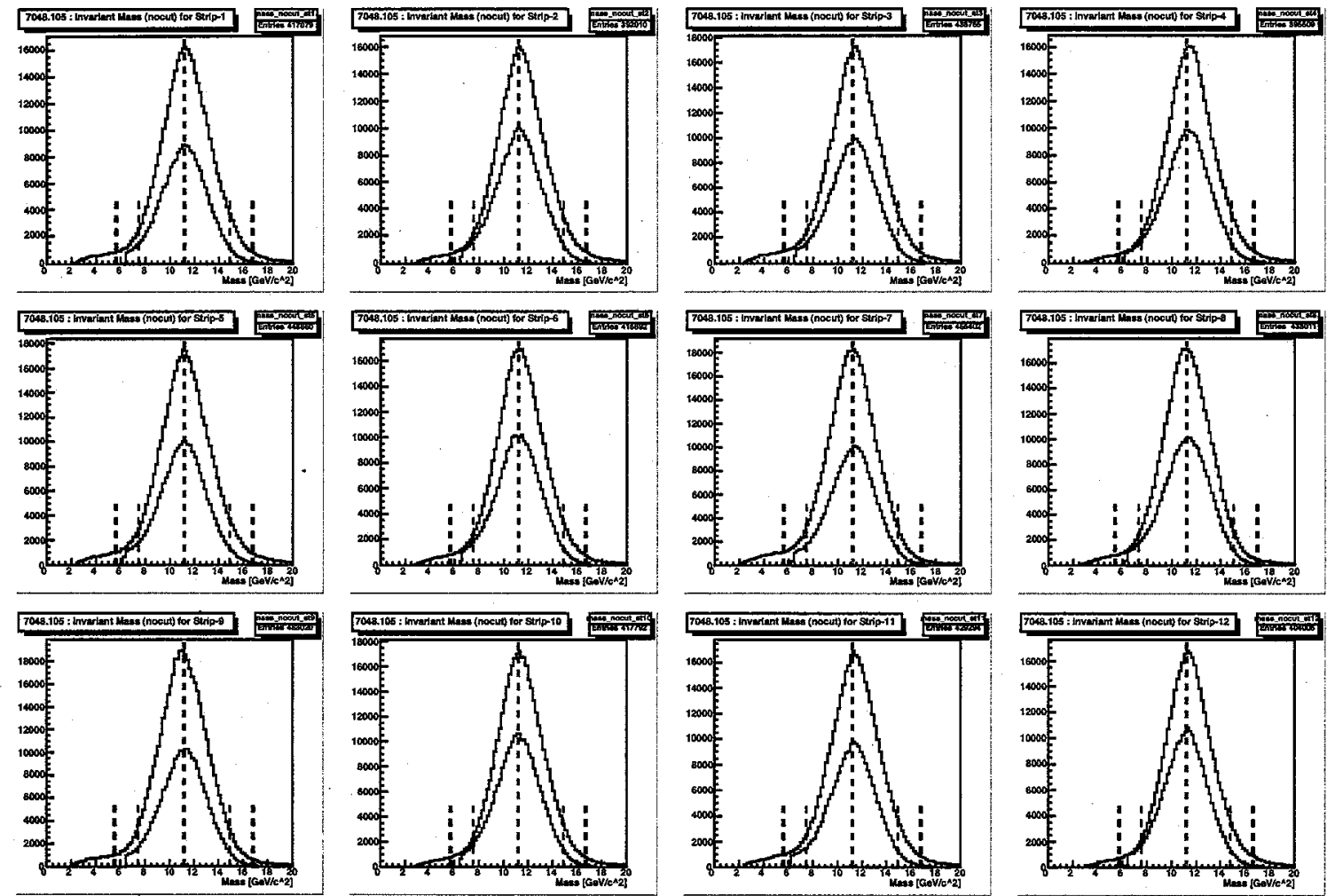

Figure 5.21: The invariant mass distributions of fill 7048 observed by the detector D1. Relatively higher backgrounds are observed in the lower side of the carbon mass peaks. 
More detailed discussions are made in Appendix. B Although it is not conclusive, the runs/fills detected by the anomaly detectors were classified into three distinct groups based on the anomaly bunch numbers appeared as summarized in Table 5.3.

Table 5.3: The three groups of anomalies found in the bunch asymmetry. (y) and (b) identifies the observed polarimeters either yellow or blue, respectively.

\begin{tabular}{cc}
\hline \hline Anomaly & Fill/Run \\
\hline $70<$ Bunch ID $<80$ & $7053(\mathrm{y}), 7054(\mathrm{y}), 7055(\mathrm{y}), 7056(\mathrm{y})$ \\
Bunch ID $<20$ & $7325.107(\mathrm{y}), 7325.102(\mathrm{~b}), 7327(\mathrm{y}), 7327(\mathrm{~b})$ \\
Diverged Bunches & $7048(\mathrm{y})$ \\
\hline \hline
\end{tabular}




\section{Chapter 6}

\section{RUN BY RUN POLARIZATION}

\subsection{Average $A_{\mathrm{N}}$}

The carbon events which passed kinematic cuts $(|\Delta M| \leq 3 \sigma$ and $400 \leq E \leq 900 \mathrm{keV})$ were then integrated over the energy range. As it is discussed in details in Section 6.2, the polarization is extracted through the strip by strip asymmetries calculated using so selected events per strip. The observed asymmetries were divided by the average $\overline{A_{\mathrm{N}}}$ to convert the asymmetry into the polarization. The $\overline{A_{\mathrm{N}}}$ is the average analyzing power within the energy range of the event selection. It is calculated by averaging $A_{\mathrm{N}}\left(E_{i}\right)$ weighted by the yields $Y[i]$ of the $i$ th bin in the energy spectrum. $E_{i}$ is the $i$ th bin of the energy $E$.

$$
\overline{A_{\mathrm{N}}}=\frac{\sum_{i}^{N} A_{\mathrm{N}}\left(E_{i}\right) \times Y[i]}{\sum_{i}^{N} Y[i]}
$$

where $N$ is the total number of bins in the energy spectrum. In Run05 analysis the the energy spectrum histogram range from zero to $1500 \mathrm{keV}$ was binned by 180 . The number of bins are 59 between the energy range $400 \leq E \leq 900 \mathrm{keV}$. $i$ runs for the maximum bins up to 1500 though, bins out side $400 \leq E \leq 900 \mathrm{keV}$ obviously do not contribute on $\overline{A_{\mathrm{N}}}$ because $Y[i]=0$.

Shown in Fig.6.1 is the typical energy spectrum of the carbon events combined for all active strips after the kinematic cuts. The curve is a model prediction[9] of $A_{\mathrm{N}}(E)$ scaled by the Run04 data.

\section{$6.2 \sin (\phi)$ fit}

The run-by-run polarization is calculated based on the strip asymmetries, combining all bunch-by-bunch asymmetries. The asymmetry of strip $i$ is calculated using the number of elastic carbon events after the kinematic cuts for all positively polarized bunches $N_{i}^{+}$and negatively polarized bunches $N_{i}^{-}$in strip $i$ : 


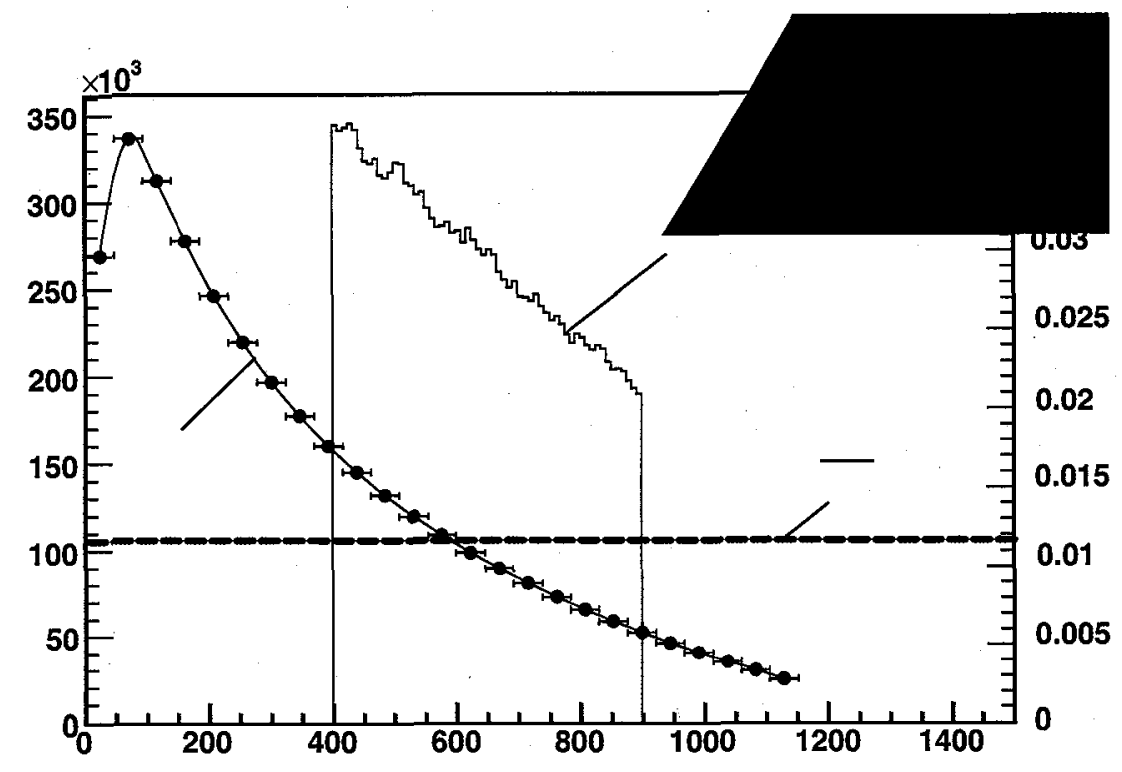

Figure 6.1: The typical energy spectrum of the carbon events combined all active strips after the kinematic cuts. The curve is a model prediction of $A_{\mathrm{N}}(E)$ scaled by the Run04 data. Dashed line shows the weighted average $\overline{A_{\mathrm{N}}}$.

$$
A_{i}=\frac{N_{i}^{+}-R_{i} N_{i}^{-}}{N_{i}^{+}+R_{i} N_{i}^{-}}
$$

where $i$ runs for active strips up to 72. The luminosity ratio for the strip $i$ is defined

$$
R_{i}=\frac{\sum_{j \neq i, 37-i, 36+i, 72-i}^{72} N_{j}^{+}}{\sum_{j \neq i, 37-i, 36+i, 72-i}^{72} N_{j}^{-}} .
$$

In order to remove the bias effect from the strip $i$ for the luminosity calculation, the strip $i$ is excluded from the luminosity calculation. Also to avoid introducing false asymmetry comes from the geometrical acceptance effect by doing so, not only the strip locates diagonally opposite location, but also ones locate at cross geometries are excluded as well. As tabulated in Table 6.1, total of 4 strips were excluded from the luminosity calculation for the asymmetry calculation of a given strip.

Plotted in Fig. 6.2 with solid circles are typical example of so calculated strip asymmetries divided by the $\overline{A_{N}}$ as a function of the azimuthal angle in the unit of radian. The coverage of the $2 \mathrm{~mm}$ strip width is translated to be $10.8 \mathrm{mrad}$ in the azimuthal angle acceptance. The central azimuthal position of strip $i$ of the detector $\mathrm{D} x$ is given:

$$
\phi_{i}=\phi_{D x}+\tan ^{-1}\left(\frac{2 i-13}{185}\right)
$$


Table 6.1: Exclusion strip combination list from the luminosity calculations. The most left column is the strip number of which asymmetry is concerned, and following 4 columns are strip numbers to be excluded from the luminosity calculations of the strip asymmetry.

\begin{tabular}{c|cccc}
\hline \hline 1 & 1 & 36 & 37 & 72 \\
2 & 2 & 35 & 38 & 71 \\
3 & 3 & 34 & 39 & 70 \\
$\ldots$ & $\ldots$ & $\ldots$ & $\ldots$ & $\ldots$ \\
36 & 36 & 1 & 72 & 37 \\
$\ldots$ & $\ldots$ & $\ldots$ & $\ldots$ & $\ldots$ \\
\hline \hline
\end{tabular}

$$
\left\{\phi_{\mathrm{D} 1}, \phi_{\mathrm{D} 2}, \phi_{\mathrm{D} 3}, \phi_{\mathrm{D} 4}, \phi_{\mathrm{D} 5}, \phi_{\mathrm{D} 6}\right\}=\{\pi / 4, \pi / 2,3 \pi / 4,5 \pi / 4,3 \pi / 2,7 \pi / 4\}
$$

where $i$ runs for strips per detector (up to 12). 185 is the distance from the target to detector in the unit of $\mathrm{mm}$ and the numerator $2 i-13$ has dimension of length as well. The observed polarization for detectors in D1, D3, D4, D6 are suppressed due to the sensitivity to the vertical polarization by $\sqrt{2}$ compared to the detectors D2 and D3.

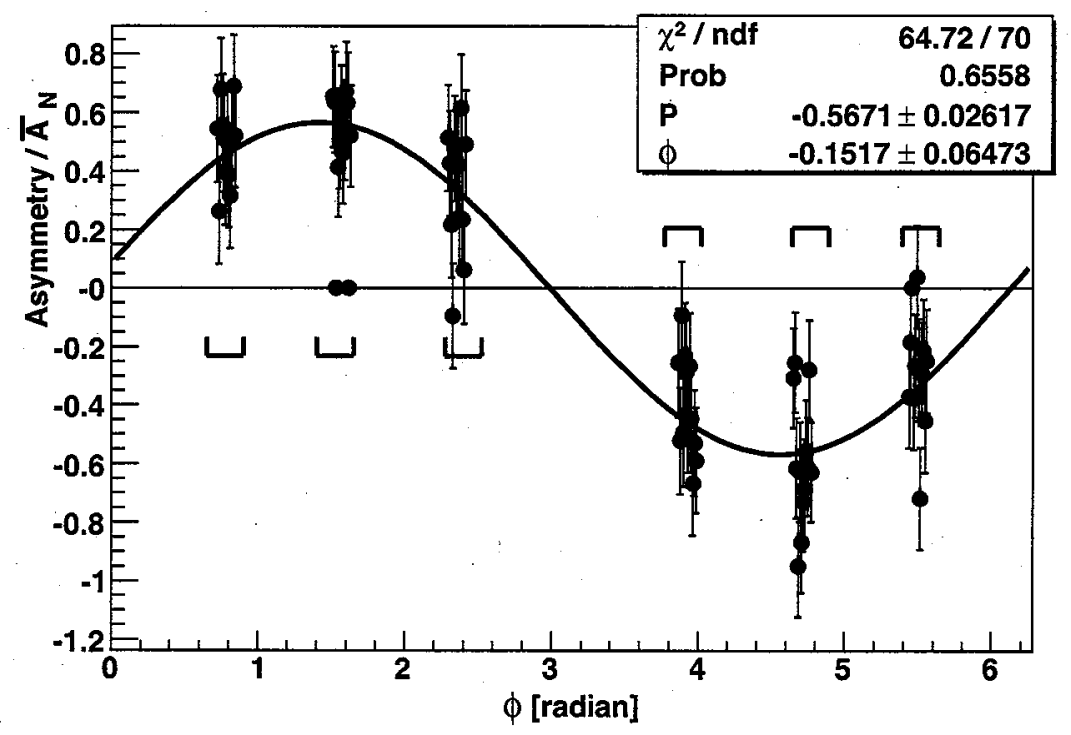

Figure 6.2: The strip by strip polarization plotted as a function of the azimuthal angle (rad). The red curve represents the best fit to the data of function 6.6 .

The strip by strip polarizations are then fitted with the sin function

$$
P(\phi)=P \sin (\phi+\Delta \phi)
$$


where $P$ is strip averaged polarization and $\Delta \phi$ is the radial polarization vector, respectively and they were set as free parameters. The best fit result is drawn by the red curve in the figure.

After disabling strips for the suspiciously behaving periods through the QA analysis as discussed previously, the $\chi^{2}$ of the fit distributes around 1 as shown in Fig. 6.3. Each entry to the histograms are $\chi^{2}$ of the fit of good measurements (survived the QA path) during Run05 in physics stores. As described in Section 5.2.3, the double elastic band were observed quite frequently during Run05, but these strips were not necessarily disabled because the effect shouldn't appear for the first order. The tiny difference in the $\chi^{2}$ distributions demonstrated that the inclusion or exclusion of these strips cause very little impact on the polarization.
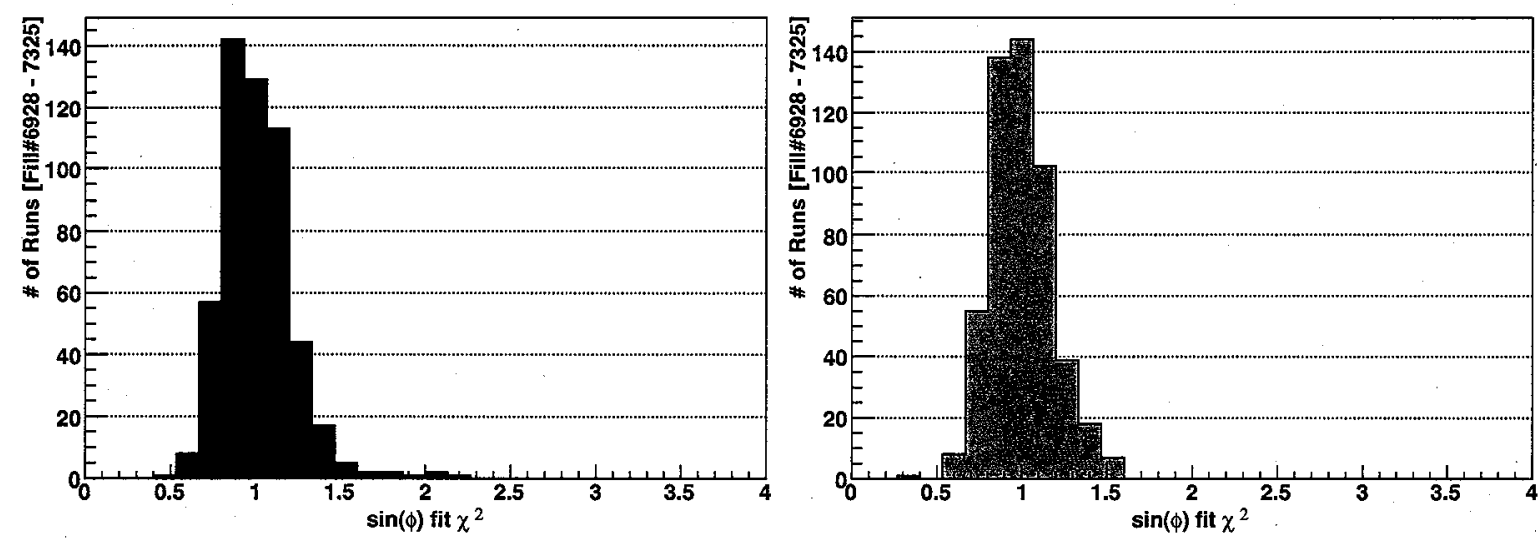

Figure 6.3: $\chi^{2}$ distribution of the azimuthal fit on the strip-by-strip polarizations applied to the all good runs in Run05. The left and right panels show the results of the blue and yellow polarimeters, respectively.

Shown in the Fig. 6.4 are the radial spin vectors obtained from the fit for all good runs. Horizontal axis is the fill number. On average, the spin vectors are off vertical by several degrees for both blue and yellow beams during Run05. 

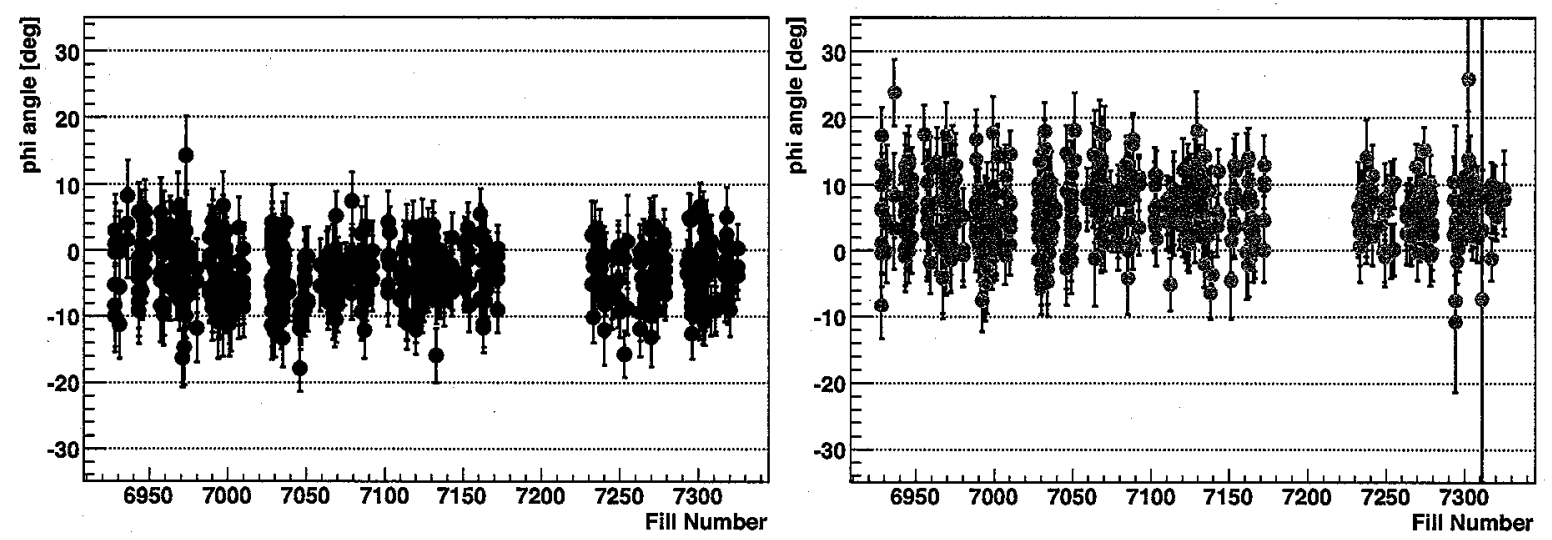

Figure 6.4: Radial spin vector $\phi$ distribution for blue (left) and yellow (right). 


\section{Chapter 7}

\section{POLARIZATION PROFILE}

The most of the polarization measurements in Run05 was done at the fixed target position, aimed to measure the polarization at the center of the beam. The typical beam size at the $100 \mathrm{GeV}$ at the location of proton-Carbon polarimeters is around $1.5 \sim 2 \mathrm{~mm}$ at FWHM. In contract to the beam spot size, the carbon ribbon target width is only $4 \sim 10 \mu \mathrm{m}$ and thus it measures only the local polarization of the beam wherever the target is positioned. On the other hand, the actual size of the H-Jet gas target is typically $6 \mathrm{~mm}$, which is large enough to cover the beam spot size at IP12. Therefore what the H-Jet measures is averaged polarization over the beam polarization profile. The intrinsic difference between protonCarbon and the H-Jet polarimeters is thus the coverage area of the finite transverse target beam spot size as illustrated in Fig. 7.1. Still the measured polarization by pC-polarimeters can be directly comparable to what the H-Jet polarimeter measures and applicable to the experiments, if there is no polarization profile in the beam. This is not the case if there is, then a profile correction needs to be applied before the average polarizations are compared between the $\mathrm{H}-\mathrm{Jet}$ and proton-Carbon polarimeters.

\subsection{Dedicated Polarization Profile Measurements}

In order to measure the polarization profile, three dedicated measurements were executed during Run05. They are two measurements using the yellow polarimeter (Fill \#7133 and \# 7151) and one using the blue polarimeter (Fill \#7151). Each profile measurement consisted of $7 \sim 13$ independent runs at different horizontal target positions in every $0.2 \sim 0.5 \mathrm{~mm}$ step as tabulated in Tables $3.1 \sim 3.3$. Each measurements were accumulated constant events $\sim$ 20 Mevents regardless of the target position. The measurements away from the beam center thus took longer time due to the low event rate. ${ }^{1}$

The resulting polarization profile for the blue beam is presented in Fig.7.2. The top panel

\footnotetext{
${ }^{1} \mathrm{~A}$ few scan profile measurements, with equal time at each target position (some used a horizontal target) were taken near the end of the 2005 run. However, these measurements showed strange odd-even strip patterns in number of events in banana cut, and therefore recognized as unreliable and unsuccessful measurements. The cause has not been understood.
} 


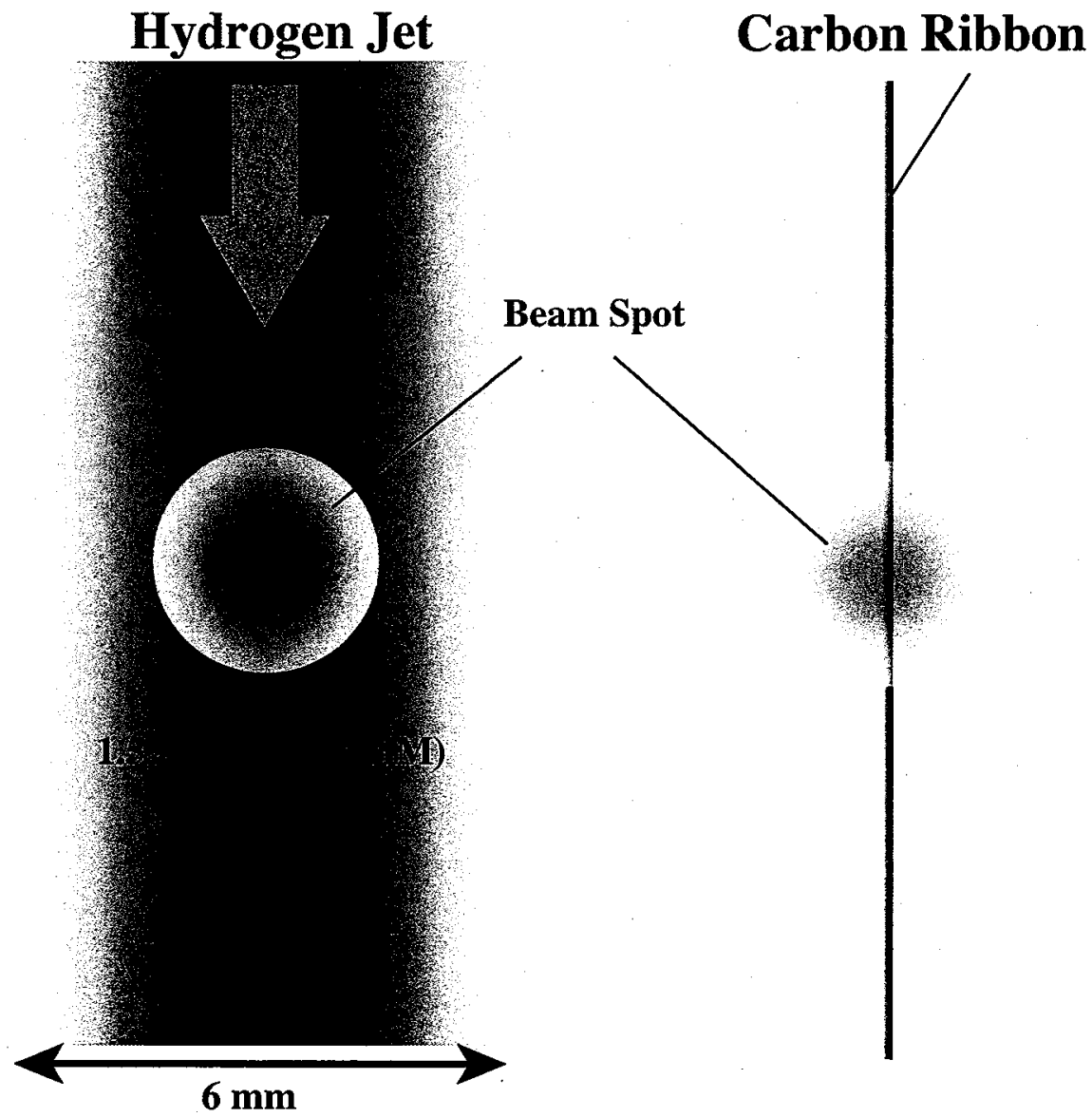

Figure 7.1: Images of the area coverage difference of the target on the beam spot between the H-Jet (left) and proton-Carbon (right) polarimeters. 
shows the polarization profile whereas the bottom panel shows the beam intensity profile, respectively. While the beam intensity profile is well reproduced by the Gaussian shape, the beam polarization profile shows reasonable $\chi^{2}$ for the linear and Gaussian functions. A small $\chi^{2}$ of $\sim 1.2$ with linear fit and large width $\sigma \sim 3 \pm 0.6 \mathrm{~mm}$ implies there was no or very weak horizontal polarization profile in blue beam when the measurement was executed during fill \# 7151 within the measured range $\pm 1.5 \mathrm{~mm}$ from the beam center.
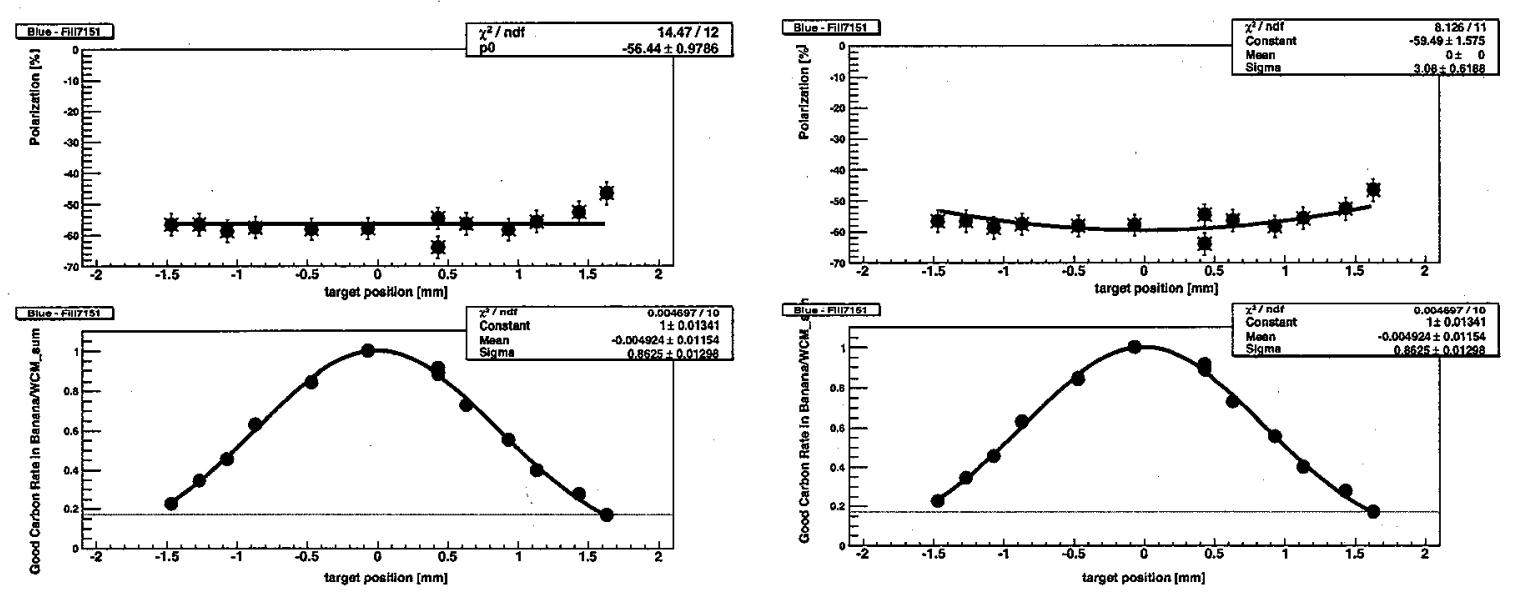

Figure 7.2: The horizontal polarization (top) and the intensity (bottom) profiles of the blue beam during fill \# 7151. The vertical axis of the intensity profile is calculated from the total number of events within the kinematic cuts divided by the wall current monitor and normalized at the peak amplitude of the Gaussian fit. The horizontal axes are the relative target position in $\mathrm{mm}$ with respect to the beam center defined by the Gaussian fit on the intensity profile. Left panel shows the linear and right panel shows the Gaussian fits on the polarization profiles, respectively.

On the contrary, the polarization profiles appeared rather strong in yellow measurements as presented in Fig. 7.3 for fill \# 7133 (left) and 7151 (right). The polarization profiles are not necessarily the same and the fill 7151 appears rather stronger than that of 7133 . The results of the linear fit on these polarization profiles are not presented in Fig.7.3, evidently they gave very poor fits. Instead they are tabulated in Table 7.1. For a comparison, the fitting results are plotted together in the same plots as shown in Fig. 7.4. Since the yellow beam polarization profiles are observed thus strong, it is necessary to somehow correct for the profile effect in order to compare the average polarizations measured by proton-Carbon and H-Jet polarimeters. Unfortunately, there are no other dedicated profile measurements reliably done for Run05 and it is still short of information to evaluate the profile correction which can be applied for all the fills.

The beam intensity profile changes from fill to fill, beginning of the fill to the end of fill. The polarization profile can be shrunk/smeared accordingly as long as it is evaluated as a function of distance from the beam center. The relevant approach to compare the profiles 

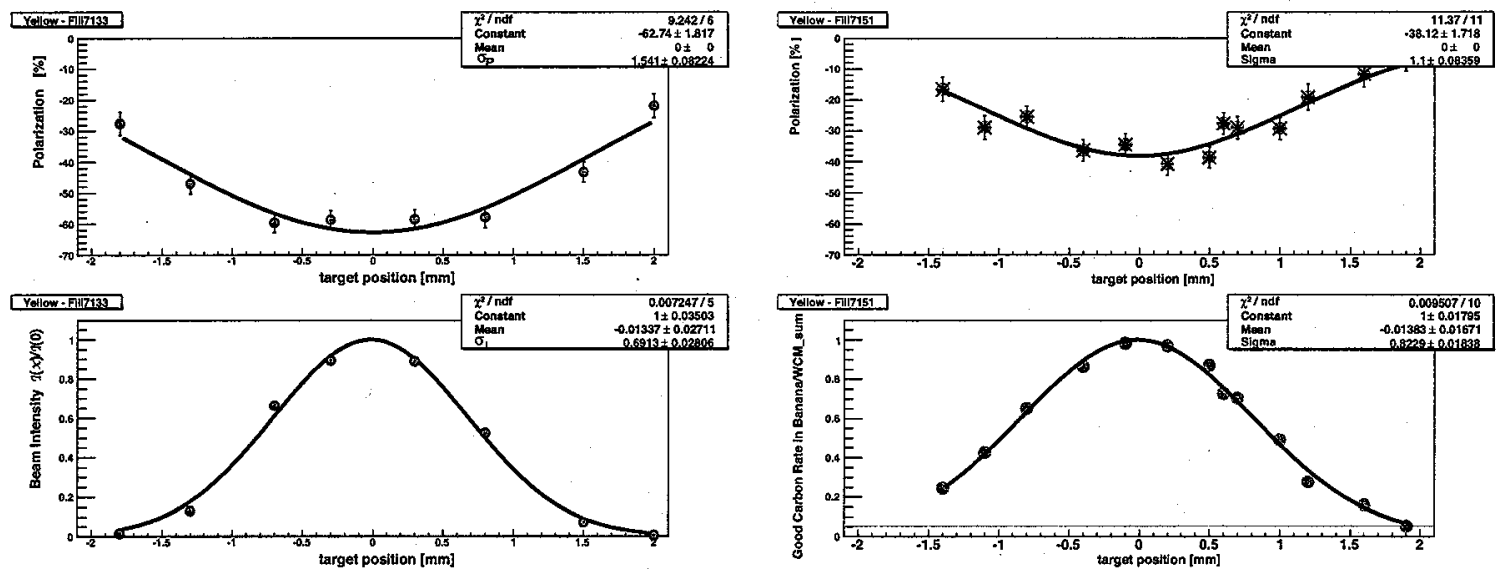

Figure 7.3: The horizontal polarization (top) and the intensity (bottom) profiles of the yellow beam during fill \# 7133 (left) and 7151 (right). Horizontal and vertical axes are the same as Fig.7.2.
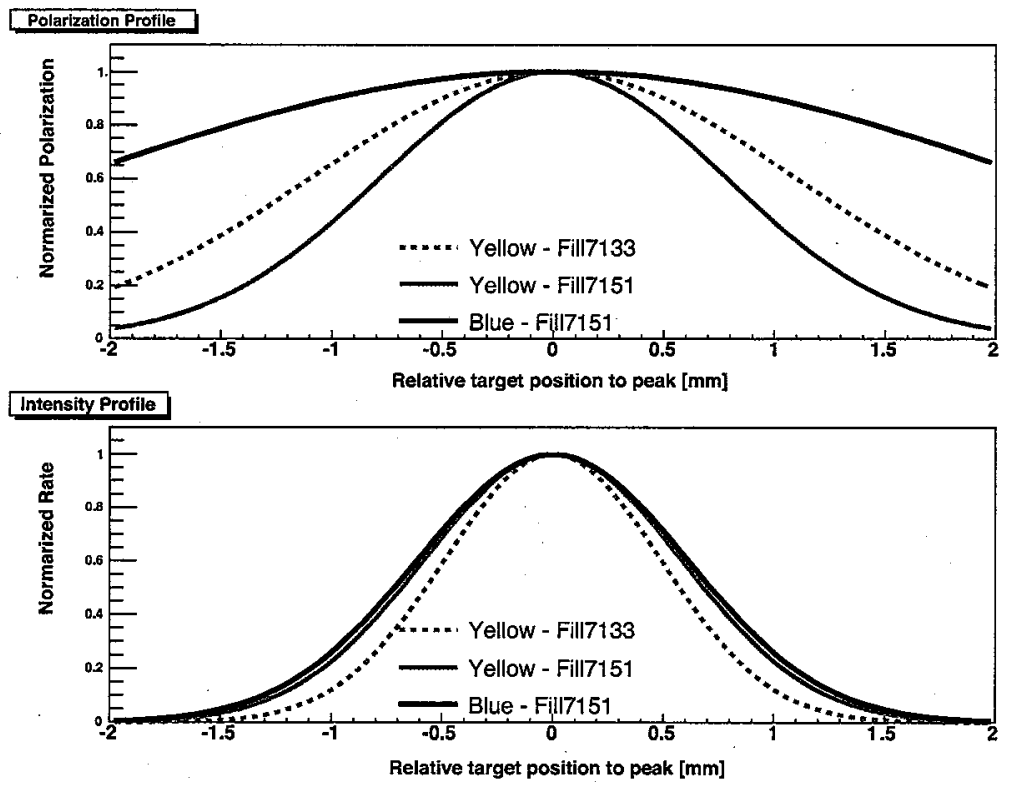

Figure 7.4: The horizontal polarization (top) and the intensity (bottom) profiles of the two measurements in yellow and one measurement in blue. The only Gaussian fitting result is plotted for blue measurement in fill \# 7151. 
Table 7.1: Profile fitting results for dedicated measurements. $\sigma_{\mathrm{I}}$ and $\sigma_{\mathrm{P}}$ are width of the intensity and polarization profiles given in the unit of $\mathrm{mm} . \chi^{2}$ per degree of freedoms are also presented for both linear and Gaussian fit cases.

\begin{tabular}{l|ccccc}
\hline \hline Beam \& (Fill) & $\sigma_{\mathrm{I}} \mathrm{mm}$ & $\sigma_{\mathrm{P}} \mathrm{mm}$ & $\left(\sigma_{\mathrm{I}} / \sigma_{\mathrm{P}}\right)^{2}$ & $\chi^{2} /$ ndf (Gaussian) & $\chi^{2} /$ ndf (linear) \\
\hline Blue (7151) & $0.86 \pm 0.01$ & $3.08 \pm 0.62$ & 0.08 & 0.6 & 1.2 \\
Yellow (7133) & $0.69 \pm 0.03$ & $1.54 \pm 0.08$ & 0.20 & 1.8 & 18 \\
Blue (7151) & $0.82 \pm 0.02$ & $1.10 \pm 0.08$ & 0.56 & 1.1 & 7.0 \\
\hline \hline
\end{tabular}

between different measurements are to correlate the profile as a function of the relative rate with respect to the peak intensity. This way the emittance difference from beam to other beam can be eliminated from the polarization profile and it is now purely defined just by the beam intensity, i.e. free from the target position. The two Gaussians of the beam intensity and the polarization profiles are given as a function of the target position $x$. $x$ is the distance from the intensity peak, not absolute target position) :

$$
\begin{aligned}
& \mathcal{I}(x)=e^{-\frac{x^{2}}{2 \sigma_{\mathrm{I}}^{2}}} \\
& \mathcal{P}(x)=e^{-\frac{x^{2}}{2 \sigma_{\mathrm{P}}^{2}}}
\end{aligned}
$$

where $\mathcal{I}(x)$ and $\mathcal{P}(x$ are the intensity and polarization profiles normalized to be 1 at their peaks, and $\sigma_{\mathrm{I}}$ and $\sigma_{\mathrm{p}}$ are width of these profiles, respectively. There are also profiles in vertical direction, but it is averaged over for this case. Eliminate $x$ from these equations then we obtain

$$
\mathcal{P}=\mathcal{I}^{2}
$$

where

$$
r=\left(\frac{\sigma_{\mathrm{I}}}{\sigma_{\mathrm{P}}}\right)
$$

Since $\mathcal{I}$ and $\mathcal{P}$ are defined as the relative intensity and the polarization with respect to the peak, they run from 0 to 1 . Thus Eq. 7.3 gives $\mathcal{P}=1$ at the peak intensity $\mathcal{I}=1$. The three polarization profiles from dedicated measurements are now characterized simply by ratio $r$ and directly comparable free from the differences in the emittance of beams and are presented in Fig.7.5.

\subsection{Global Polarization Profile}

The polarization profile are subject to change from a fill to another fill, from the beginning of a fill to the end of the fill. Thus it is ideal to measure the profile frequently enough to 


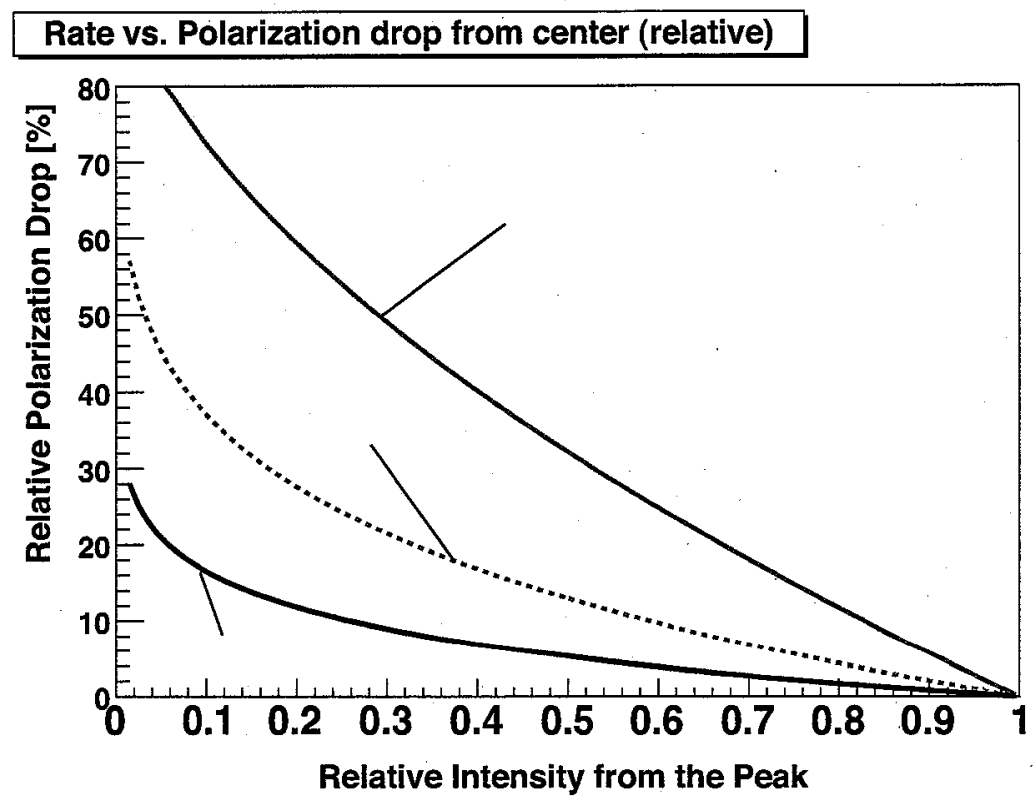

Figure 7.5: Three dedicated polarization profiles are compared in the definition of Eq.7.3. The vertical axis is $(1-\mathcal{P}) / \mathcal{P} \times 100$.

apply the profile correction to observed peak polarizations accordingly. In reality, we did not have such profile measurements sufficient enough to identify the profiles dynamically alters in Run05. In this section, we evaluate the average profile which can be applied to all Run05 data for a finite accuracy instead.

In principle, a regular polarization measurement using the proton-Carbon polarimeter was aimed to insert the target at the center of the beam and was meant to measure the peak polarization of the beam profile. However, the target was not necessarily always placed at the center of the beam due to somewhat loose control of the target position and happen to measure polarizations at off centered locations. Since we have the independent measurement of the beam intensity using the wall current monitor (WCM)[16], we would know the expected event rate to be observed by the polarimeters for the given thickness of a target. We do not know the accurate thickness of the target where the beam hit, so we do not have accurate prediction of the rate neither. However we at least have good guess of the relative position of the target once we somehow know the maximum rate at the peak is.

Shown in Fig. 7.6 are event rates of each proton-Carbon measurement plotted as a function of the time from the first measurement at the stored energy of a given fill. The event rate is defined by the total number of carbon events in the kinematic cuts (all active strips are combined) divided by the beam intensity (sum of all active bunches) measured by the wall current monitor. Since it is normalized by the beam intensity, the event rate is the number of events per $\left(10^{11}\right)$ protons and therefore should be constant from a run to another run as long as the target and the emittance are same. Since the emittance doesn't change 
drastically in the short period, any drastical fluctuation of the event rate can be interpreted as a consequence of the altering target position with respect to the beam center.
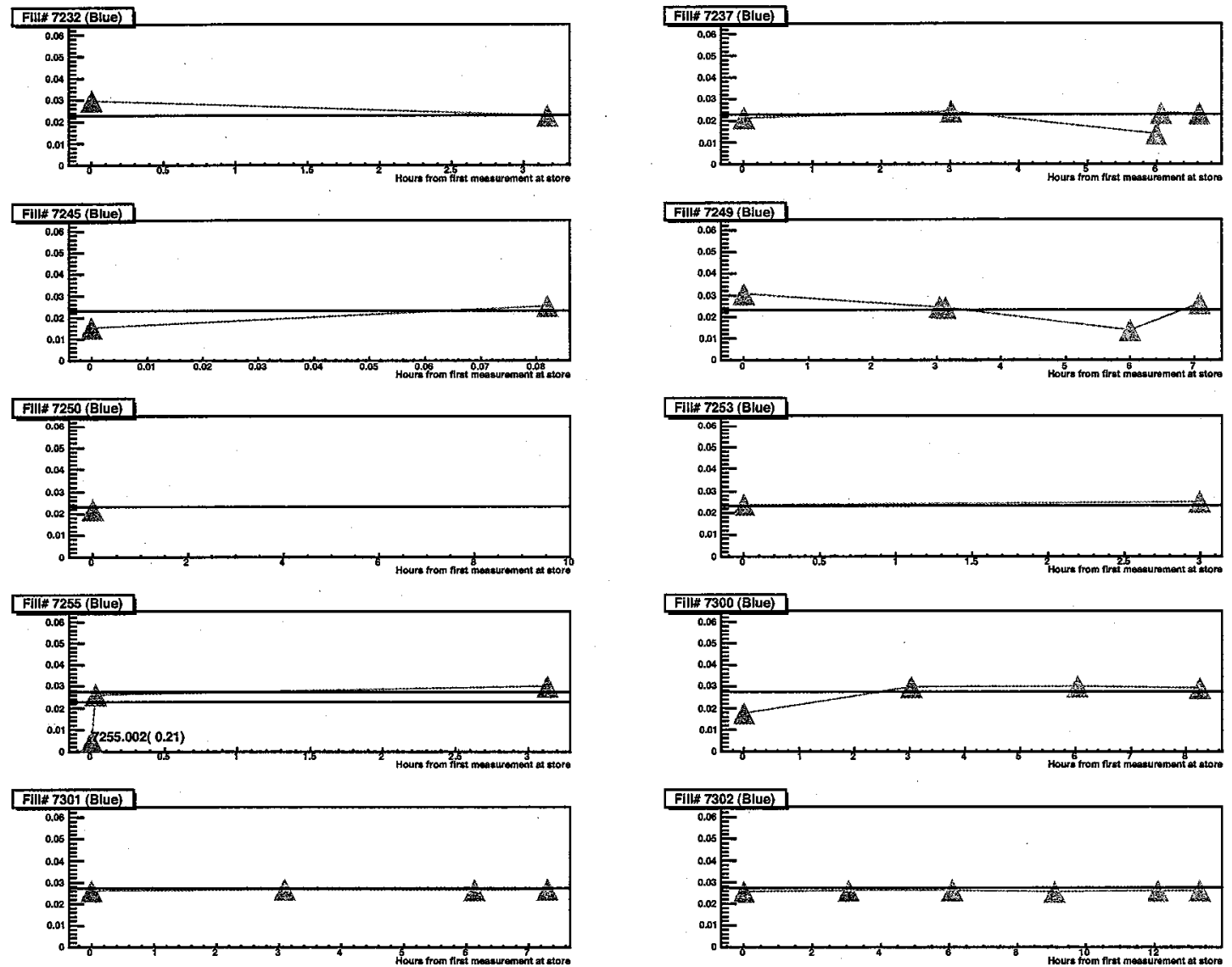

Figure 7.6: Event rates of each measurement using the blue polarimeter divided by the beam intensity measured by the wall current monitor plotted as a function of the time from the first measurement of a given fill. The units of axis are [events $/ 10^{11}$ protons] (vertical) and hour (horizontal).

Shown in Fig. 7.7 and Fig. 7.8 are the event rate distributions classified for different target periods observed by the blue and yellow polarimeters, respectively. The blue measurements are classified into three different target periods whereas yellow ones are six of them. Gaussian fits are applied for each event rate distribution and its mean was interpreted as the expected peak event rate at the center of the beam for the given target period. The measurements below $60 \%$ of the peak rate were only quoted to determine the polarization profile as is discussed following though, they were excluded from neither the average polarization for the $\mathrm{H}$-Jet normalization nor the fill-by-fill polarization provided to the experiments.

Once the expected rate at the peak is determined for a given target, here we introduce the universal rate which is defined by the relative rate with respect to the expected rate at the 

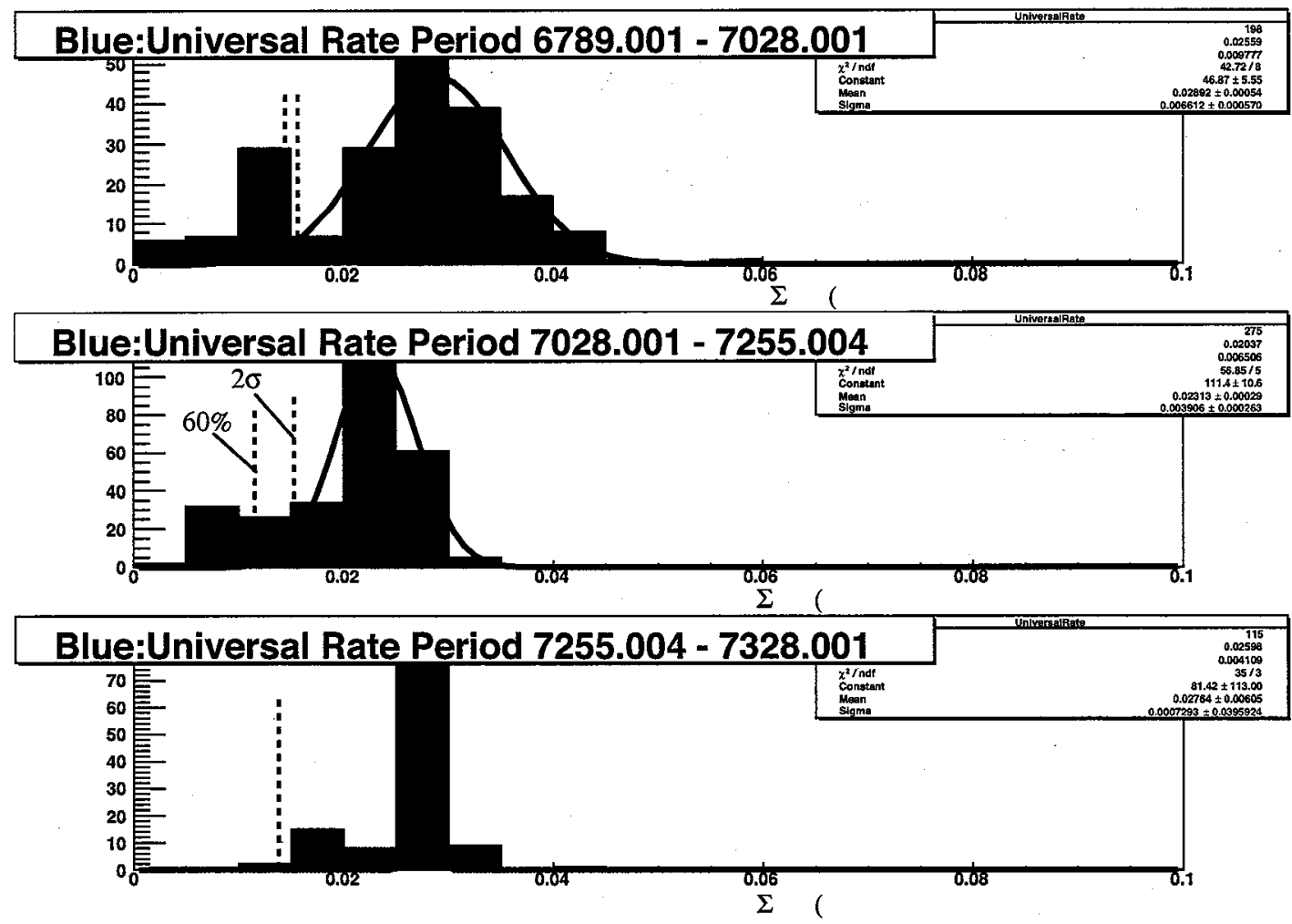

Figure 7.7: The event rate distribution of the blue proton-Carbon runs classified for the three different target period. The vertical dashed lines indicates $2 \sigma$ from the peak and 0.6 of the peak rate, respectively. 


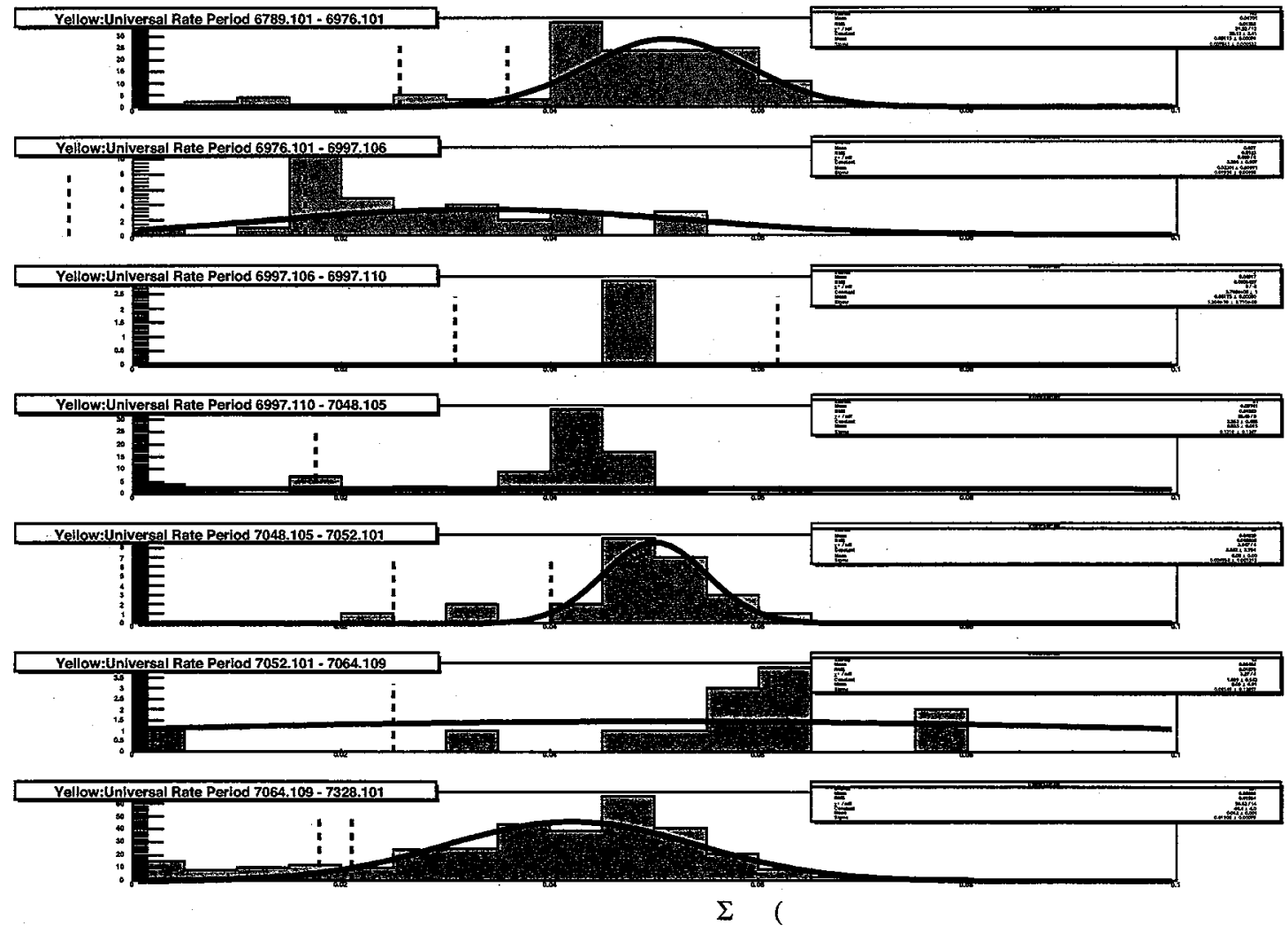

Figure 7.8: The event rate distribution of the yellow proton-Carbon runs classified for the three different target period. 
peak. By definition, the universal rate supposed to run from 0 to 1 , however it extends above 1 simply because the peak rate was estimated from the mean of the event rate distributions as demonstrated in Fig. 7.7 and Fig. 7.8. There are measurements observed higher rates than the expected higher rates, and they appear above 1 in the universal rate. Now measurements taken using different targets can be combined under the new definition of rate, i.e. universal rate. Shown in Fig.7.9 is the universal rate distribution of the blue polarimeter. Data in three different target periods are all combined under the new rate definition.

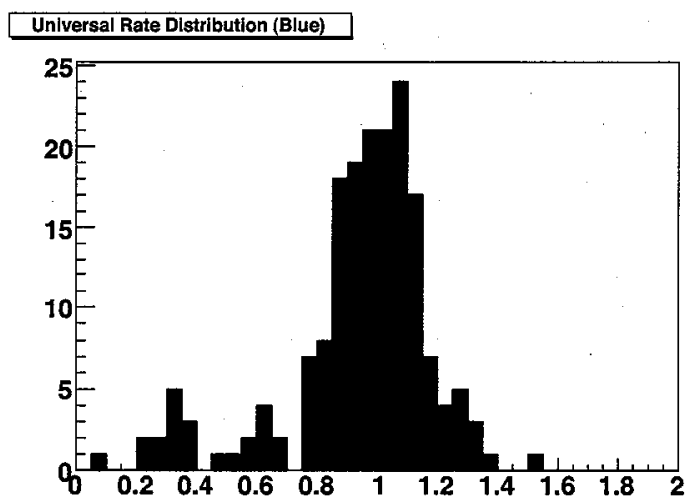

Figure 7.9: The universal rate distribution for blue polarimeter throughout Run05 measurements.

Once the universal rate is defined which allows us to correlate the observed polarizations with rate regardless of different targets used. Presented in Fig. 7.10 are the correlation between the observed polarizations and the universal rate for the blue (left) and yellow (right) measurements, respectively. The black solid lines are the best fit to the data in the universal rate region from 0 to 1 with Eq. 7.3, because the profile formula becomes meaningless in the region beyond 1 . The universal rate is equivalent with the relative intensity $\mathcal{I}$, which was introduced in section. 7.1. As you can see, the polarization profile for the blue data indicates almost no profile which is consistent with the study made in section. 7.1. On the other hand, the yellow data demonstrate strong correlation between polarizations and $\mathcal{I}$ (or rate) which is also consistent with the previous study. However, the average profiles are somewhat weaker than the Gaussian fit results on the three dedicated profile measurements in both blue and yellow cases. In other word, these three dedicated measurements are stronger than the average, but they are still in the distribution of the entire data sets. Thus we employ the best fit values and they are summarized in Table. 7.2.

\subsection{Profile Correction}

Because the proton-Carbon polarimeter automatically averages over the vertical polarization profile, it is only required to make correction for the horizontal profile. In this section, the 

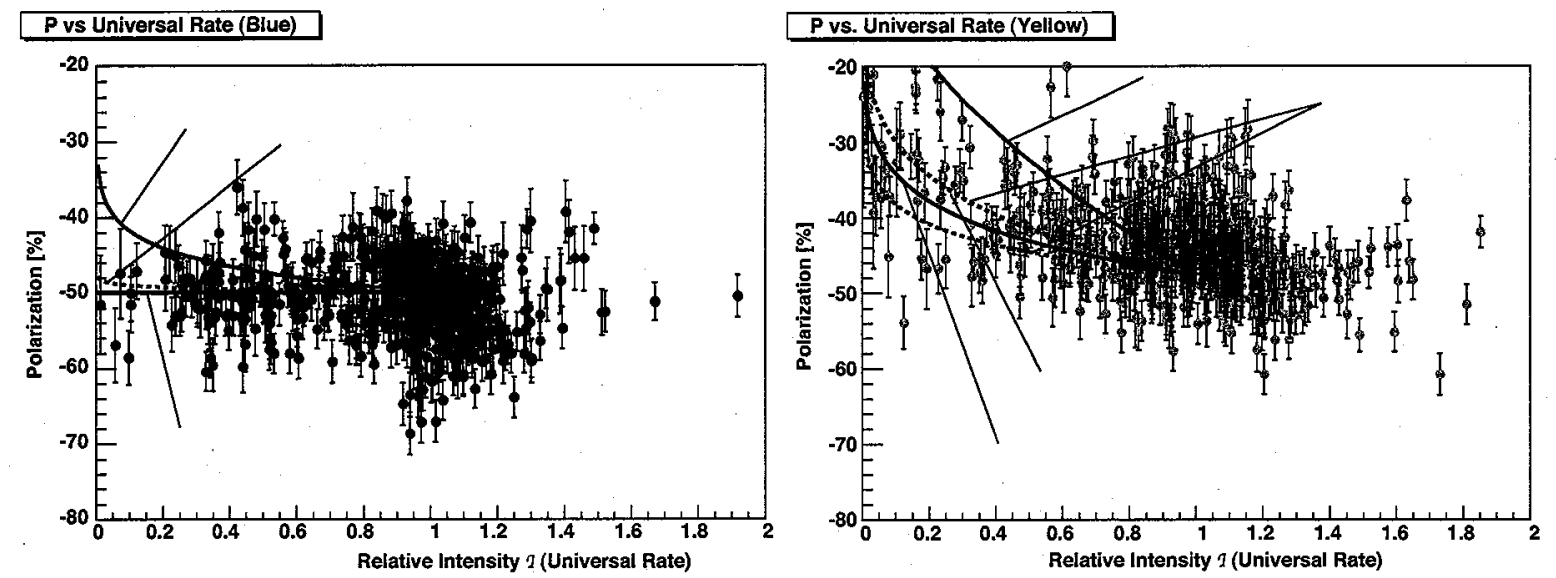

Figure 7.10: Universal Rate vs. polarization for blue (left) and yellow (right). Black solid lines are the best fit to the data in the rate region between 0 to 1 using Eq. 7.3. The result of profiles observed by the dedicated polarization profile measurements are also plotted. The relative $\mathcal{P}$ are scaled by the average polarization $\overline{P(\mathcal{I})}$ of the best fit at $\mathcal{I}=0$. The dashed lines are upper and lower bound of the error assigned to the best fit. The yellow profile measurement of fill \# 7133 is overlapping with the upper bound of this error band.

Table 7.2: The best fit results on the polarization and the intensity correlation plots for blue and yellow beams with a statistical $\Delta r_{\text {sta }}$,a model $\Delta r_{\text {model }}$, systematic (binning) $\Delta r_{\text {sys }}$, and total $\Delta r_{\text {tot }}$ errors. The error only apply for positive for blue beam.

\begin{tabular}{l|ccccc}
\hline \hline Beam & $r$ & $\Delta r_{\text {sta }}$ & $\Delta r_{\text {model }}$ & $\Delta r_{\text {sys }}$ & $\Delta r_{\text {tot }}$ \\
\hline Blue & 0.00 & 0.09 & 0.00 & 0.00 & 0.09 \\
Yellow & 0.38 & 0.04 & 0.04 & 0.04 & 0.07 \\
\hline \hline
\end{tabular}

correction factors are calculated based on the average polarization profiles evaluated in the section. 7.2. The correction factor $C_{1 \mathrm{x}}$ can be calculated from the average of the polarization profile $\mathcal{P}(x)$ (Eq. 7.2) weighted by the beam intensity profile $\mathcal{I}(x)$ (Eq. 7.1):

$$
\begin{aligned}
C_{1 \mathrm{X}} & =\frac{\int_{-\infty}^{\infty} d x \cdot \mathcal{P}(x) \cdot \mathcal{I}(x)}{\int_{-\infty}^{\infty} d x \cdot \mathcal{I}(x)} \\
& =\frac{\int_{-\infty}^{\infty} d x \cdot e^{-\frac{x^{2}}{2 \sigma_{\mathrm{I}}^{2}}} \cdot e^{-\frac{x^{2}}{2 \sigma_{\mathrm{P}}^{2}}}}{\int_{-\infty}^{\infty} d x \cdot e^{-\frac{x^{2}}{2 \sigma_{\mathrm{I}}^{2}}}} \\
& =\frac{1}{\sqrt{1+\left(\frac{\sigma_{\mathrm{I}}}{\sigma_{\mathrm{P}}}\right)^{2}}}
\end{aligned}
$$


where the ratio $\sigma_{\mathrm{I}} / \sigma_{\mathrm{P}}$ is substituted by $r$ in Eq.7.4, the correction factor $C_{1 \mathrm{X}}$ can be given only by the simple function of $r$ :

$$
C_{1 \mathrm{X}}=\frac{1}{\sqrt{1+r^{2}}}
$$

The subscript $1 \mathrm{X}$ refers to weighting by the intensity to the power 1 , in the horizontal dimension. For obtained $r$ as tabulated in Table. 7.2, the resulting correction factor for the horizontal beam profile is tabulated in Table. 7.3. The polarizations measured by protonCarbon using vertical target $\overline{P_{k}^{\mathrm{pC}}}$ and the $\mathrm{H}$-Jet $\overline{P_{k}^{\mathrm{H}-\mathrm{Jet}}}$ polarimeters of the H-Jet operation period $k$ are thus related as

$$
\overline{P_{k}^{\mathrm{H}-\mathrm{Jet}}}=\overline{P_{k}^{\mathrm{pC}}} C_{1 \mathrm{X}}
$$

Table 7.3: The horizontal polarization profile correction factors $C_{1 \mathrm{X}}$ for the blue and yellow beams. The error $\Delta C_{1 x}$ only applies to negative for the blue correction, because the formula Eq. 7.8 becomes unphysical beyond $C_{1 \mathrm{x}}>1$.

\begin{tabular}{l|cc}
\hline \hline Beam & $r$ & $C_{1 \mathrm{X}} \pm \Delta C_{1 \mathrm{X}}$ \\
\hline Blue & $0.00+0.09$ & $1.000-0.005$ \\
Yellow & $0.38 \pm 0.07$ & $0.935 \pm 0.022$ \\
\hline \hline
\end{tabular}

